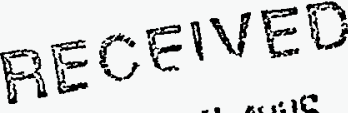

FER $\bigcirc 81450$

OSTI

\title{
BASELINE RISK ASSESSMENT OF Ground WATER Contamination At THE URANIUM MiLL TAILINGS Site Near Canonsburg, PenNSYLVANIA
}

November 1995

This report was prepared as an account of work sponsored by an agency of the United States Government. Neither the United States Government nor any agency thereof, nor any of their employees, makes any warranty, express or implied, or assumes any legal liability or responsibility for the accuracy, completeness, or usefulness of any information, apparatus, product, or process disclosed, or represents that its use would not infringe privately owned rights. Reference herein to any specific commercial product, process, or service by trade name, trademark, manufacturer, or otherwise does not necessarily constitute or imply its endorsement, recommendation, or favoring by the United States Government or any agency thereof. The views and opinions of authors expressed berein do not necessarily state or reflect those of the United States Government or any agency thereof.

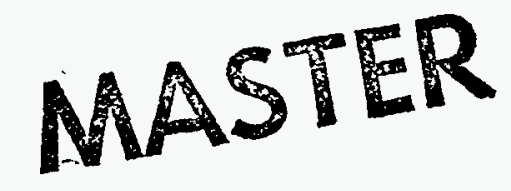




\section{INTENDED FOR PUBLIC RELEASE}

This report has been reproduced from the best available copy. Available in paper copy and microfiche

Number of pages in this report: 140

DOE and DOE contractors can obtain copies of this report from:

Office of Scientific and Technical Information

P.O. Box 62

Oak Ridge, TN 37831

(615) 576-8401

This report is publicly available from:

National Technical Information Service

Department of Commerce

5285 Port Royal Road

Springfield, VA 22161

(703) $487-4650$ 
Prepared for

U.S. Department of Energy Environmental Restoration Division UMTRA Project Team Albuquerque, New Mexico

Prepared by Jacobs Engineering Group Inc. Albuquerque, New Mexico

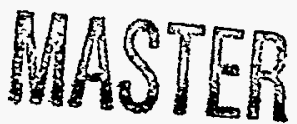

DISTRBSUTION OF THS DOCUNEAT IS LWLMUTEO 


\section{CITIZENS' SUMMARY}

\section{INTRODUCTION}

The Uranium Mill Tailings Remedial Action (UMTRA) Project consists of two phases. Phase $\mathrm{I}$ is the Surface Project, and phase II is the Ground Water Project.

For the UMTRA Project site located near Canonsburg, Pennsylvania (the Canonsburg site), the Surface Project cleanup occurred from 1983 to 1985 , and involved removing the uranium processing mill tailings and radioactively contaminated soils and materials from their original locations and placing them in a disposal cell located on the former Canonsburg uranium mill site. This disposal cell is designed to minimize radiation emissions and further contamination of ground water beneath the site.

The Ground Water Project will evaluate the nature and the extent of ground water contamination resulting from uranium processing at the former Canonsburg uranium mill site, and will determine a ground water strategy for complying with the U.S. Environmental Protection Agency's (EPA) ground water standards established for the UMTRA Project. This ground water strategy must protect public health and the environment from radiological and nonradiological hazards. A risk assessment is one of the tools used to evaluate these hazards.

A risk assessment describes the source of uranium processing contamination and shows how people and the environment could be exposed to the contamination. As part of the risk assessment, the amount of contamination exposure is calculated and the result is used to characterize the possible public health or environmental effects that could result from this exposure.

The Canonsburg site consists of the disposal site area and Area C. Access to the disposal site area has been permanently restricted so Area $C$ is the only part of the Canonsburg site being considered for future public use. Consequently, the potential health risk resulting from possible use of ground water beneath only Area $C$ have been evaluated. Thus, when reference is made to "the Canonsburg site," the term "site" refers to Area C.

For the Canonsburg site, an evaluation was made to determine whether exposure to ground water contaminated by uranium processing could affect people's health. This exposure could occur from drinking water pumped from a hypothetical well drilled in the contaminated ground water area. Risks to public health and the environment could also result from people, plants, or animals being exposed to any surface water that has mixed with the contaminated ground water.

This risk assessment report is the first site-specific document prepared for the UMTRA Ground Water Project at the Canonsburg site. The results of this report and further site characterization of the Canonsburg site will be used to determine how to protect public health and the environment, and how to comply with the EPA standards. 


\section{RISK SUMMARY}

There are currently no human health risks associated with the contaminated ground water at the Canonsburg site because no one is using the water for domestic purposes. In addition, no one is using the contaminated ground water for irrigation. This favorable risk situation will continue if land and water uses on the site do not change. Changes of land and water uses may or may not create future human health and environmental risks. When specific future land and water uses are determined for the Canonsburg site, these uses should be evaluated to identify potential health and environmental risks from the contaminated ground water.

Contaminated ground water probably discharges into Chartiers Creek. However, swimming, fishing, or wading would not be considered as human health risks because the contamination is diluted by the flowing creek water to near background levels.

Because future land and water uses at the Canonsburg site are not known, this risk assessment evaluates a hypothetical worst-case future scenario of a hypothetical well drilled in the most contaminated portion of the aquifer beneath the Canonsburg site. In this hypothetical future scenario, the hypothetical water well would be the only source of drinking water for people and wildlife, and the only source of water for crop irrigation. Drinking this contaminated ground water could cause health problems for these people. Crops could also be harmed by the contaminated ground water. Based on the findings of this risk assessment, the contaminated ground water beneath the Canonsburg site should not be used. In addition, before any other Canonsburg site ground water is used, its possible effects should first be evaluated.

\section{GROUND WATER QUALITY}

\section{Background ground water quality}

Background ground water quality is the quality of water that would exist in the Canonsburg site area if uranium milling had never occurred. Ground water occurs in the uppermost aquifer (the upper aquifer) at approximately 3 feet (ft) (1 meter [m]) beneath the land surface. The upper aquifer consists of a sequence of unconsolidated materials overlying shallow bedrock and is underlined by a deep bedrock aquifer. Within the upper aquifer, ground water in the unconsolidated materials moves into the shallow bedrock through cracks in the rock, but it does not move into the deep bedrock aquifer because these two aquifers are separated by a low permeability rock formation. The upper aquifer is not considered a potable water resource, because it contains very little water and the water quality is naturally poor. Potable water is obtained from surface water sources or from ground water at depths greater than $100 \mathrm{ft}(30 \mathrm{~m})$ below the ground surface.

\section{Site-related ground water quality}

The main contaminants in ground water in the upper aquifer beneath the Canonsburg site are manganese, molybdenum, and uranium. The contamination appears to be confined within the area bordered by Chartiers Creek. If any contaminated ground water discharges into Chartiers Creek, it is quickly diluted to near background levels. 


\section{HUMAN HEALTH RISK ASSESSMENT}

\section{Methods}

A risk assessment begins by identifying constituents present in ground water contaminated by the uranium milling process. First, water quality in wells drilled on the Canonsburg site is compared to water quality in wells from the background area. Second, possible human health problems resulting from drinking the water containing these constituents are evaluated. Factors such as eating garden produce irrigated with this water, incidental ingestion of water and sediment while swimming in the Chartiers Creek, or consumption of fish from the creek are also considered.

This risk assessment evaluates both current and possible future health risks. To evaluate possible current risks, it must be determined whether anyone is now drinking the contaminated ground water or swimming, wading, or fishing in the Chartiers Creek. To evaluate possible future human risks, it is assumed that the sole source of water for drinking or irrigating is from a hypothetical well drilled in the most contaminated area beneath the Canonsburg site.

Health risks, other than cancer, were evaluated for children because the ratio of contaminants to body weight is the greatest for children. Children 1 to 10 years old are the most likely population group to experience health problems from drinking contaminated water. To estimate cancer risks, a lifetime exposure was assumed and these risks were evaluated for adults.

Possible health effects vary in seriousness because of several factors. The levels of contaminants in ground water vary from one well sampling to the next; people vary in their body weight, how much water they drink, and how their body reacts to chemical exposure. Whenever possible, all of these differences are considered in this risk assessment.

This risk assessment provides a series of graphs showing the different exposure levels that might occur and the most current scientific information on the types of health effects that may result from this hypothetical exposure.

\section{Results}

No drinking water or irrigation wells exist in the contaminated ground water beneath the Canonsburg site. Therefore, there are currently no associated health problems because no one uses this ground water for these purposes. This favorable situation will remain the same in the future if there are no changes in land and water uses at the Canonsburg site. Furthermore, based on existing data, no health problems would be expected to occur from people swimming or wading in Chartiers Creek, or from people eating fish caught in the creek.

Additionally, in the future, it is unlikely that people will ever use the contaminated ground water for drinking. The upper aquifer is not considered as a potable water resource in the 
Canonsburg site area, and good quality water is available from the municipal water supply system.

If anyone used the contaminated ground water for drinking, based on the concentration of contaminants found in the most contaminated wells, there are possible human health risks. Table CS.1 provides information on the nature of possible health problems that could be expected.

Table CS.1 Hypothetical future human health effects from drinking contaminated ground water from the Canonsburg site

\begin{tabular}{lcl} 
& \multicolumn{2}{c}{ Possible effects from drinking water from the upper aquifer } \\
\cline { 2 - 3 } Contaminant & Short-term & \multicolumn{1}{c}{ Long-term } \\
\hline Manganese & None & Neurological symptoms include memory \\
& & loss, irritability, muscle rigidity, tremors. \\
Molybdenum & None & None \\
Uranium & None & None \\
\hline
\end{tabular}

${ }^{\mathrm{a}}$ The effects could vary from person to person depending on the amount of water a person drinks, body weight, dietary habits, and individual sensitivities such as the preexisting kidney, liver, or heart diseases, and other factors.

Only people whose sole source of drinking water comes from a hypothetical well placed in the most contaminated ground water could be expected to experience the health problems described in Table CS.1. Consequently, the table provides the upper limit of possible risks; real future risks would probably be lower.

Manganese is the only possible health hazard in the contaminated ground water of the upper aquifer. Manganese ingestion in the amounts found in the contaminated ground water could affect the nervous system, causing memory loss, muscle rigidity, or tremors.

People's health would not be affected from eating homegrown garden produce irrigated with this water.

\section{ECOLOGICAL RISK ASSESSMENT}

\section{Methods}

The ecological risk assessment presented in this document is a screening level assessment that relies on limited environmental sampling and literature. The field of ecological risk assessment has many uncertainties because of limited scientific knowledge. Information is lacking on how some chemicals affect plants and animals. Also, the impact on plants and animals of a mixture of chemicals is poorly understood. 
The possible effects of the contaminants on wildlife and agricultural crops are being evaluated. This evaluation is done by comparing the concentration values of contaminants in the most contaminated ground water from the upper aquifer with available guideline values from regulatory agencies and related literature. The contaminants present in surface water and in sediment from Chartiers Creek are evaluated because the Canonsburg site contaminated ground water discharges into Chartiers Creek. The assumption that plant roots extend into the upper aquifer and take up the most contaminated ground water from the upper aquifer is also evaluated.

\section{Agricultural results}

There are currently no risks to crops from the contaminated ground water beneath the Canonsburg site because crops are not irrigated with that water. If, in the future, the ground water beneath the most contaminated part of the Canonsburg site were used as a sole irrigation water source, manganese could harm manganese-sensitive plants such as spinach and soybean.

\section{Ecological results}

No ecological risks exist to plants at harvestable maturity whose roots may have extended into soils containing contaminated ground water.

Limited environmental sampling and available guidelines from literature and regulatory agencies were not sufficient to fully evaluate possible long-term impacts of the affected ground water or surface water on plants or animals.

\section{CONCLUSIONS}

Ground water contamination beneath the Canonsburg site is limited to the uppermost aquifer. The contamination appears to be confined within the Canonsburg site area. Chartiers Creek probably creates a barrier for this contamination. Currently, there are no drinking water or irrigation wells drilled into the contaminated ground water at the Canonsburg site. Consequently, based on existing data, there are no current human health risks associated with the Canonsburg site contaminated ground water.

This risk assessment has determined that there could be certain health problems in people if, in the future, contaminated ground water were used for drinking. Therefore, no one should use contaminated ground water for this purpose. Crops irrigated with contaminated ground water could also be adversely affected. Consequently, contaminated ground water should not be used for irrigation.

The Canonsburg site evaluation is ongoing and will include further study of ground water quality, water levels, and ground water movement. In addition, sampling of surface water and sediment will be considered in future work plans. This risk assessment and future investigations will be used to determine how to deal with the contaminated ground water. In addition, if specific plans for land and water uses at the Canonsburg site are determined, the possible risks from those uses should be evaluated. 


\section{Section}

Page

CITIZENS' SUMMARY CS-1

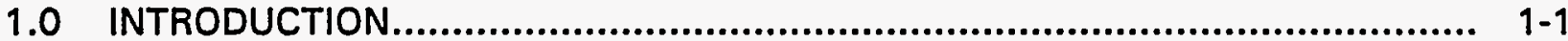

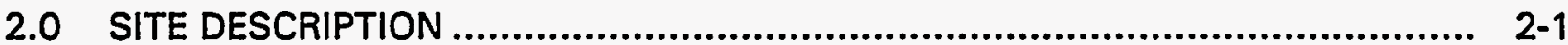

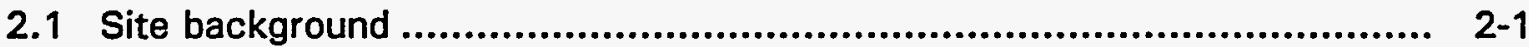

2.2 Climate................................................................................... 2-6

2.3 Physiographic setting ............................................................. 2-6

2.4 Hydrogeology ............................................................................... $2-6$

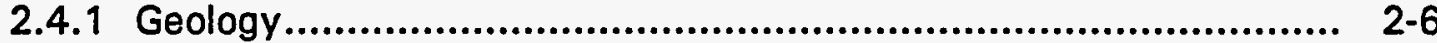

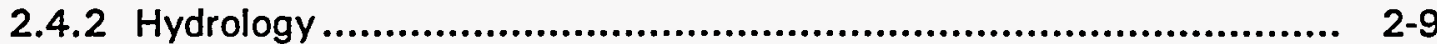

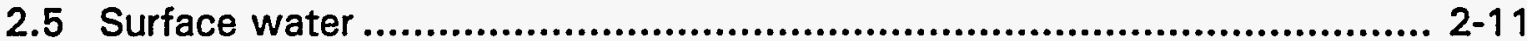

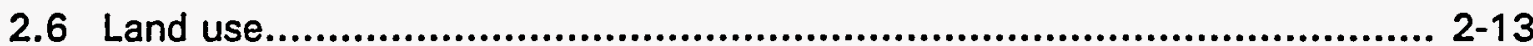

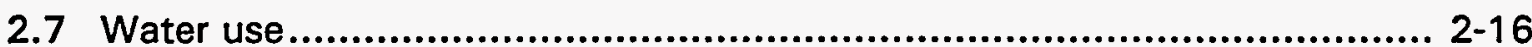

3.0 EXTENT OF CONTAMINATION ....................................................... $3-1$

3.1 Background ground water quality data summary ............................... $3-1$

3.2 Magnitude and extent of site-related ground water contamination............ 3-4

3.2.1 Unconsolidated materials...................................................... $3-4$

3.2.2 Bedrock unit .............................................................. 3-15

3.2.3 Summary of site-related contamination ................................... 3-15

3.3 Contaminants of potential concern ................................................. 3-16

3.4 Contaminant fate and transport...................................................... 3-16

3.5 Surface water monitoring ............................................................... 3-22

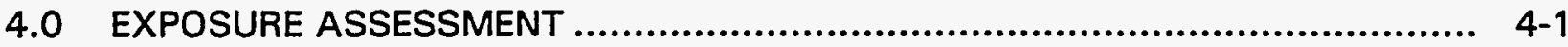

4.1 Potentially exposed population .................................................. 4-1

4.2 Exposure pathways.................................................................. $4-2$

4.2.1 Ground water............................................................... 4-2

4.2.2 Surface water/sediment ................................................... 4-6

4.3 Exposure concentrations ........................................................... 4-8

4.4 Estimation of ground water ingestion intake ...................................... 4-11

4.5 Exposure assessment uncertainties................................................... 4-18

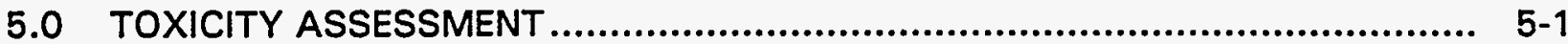

5.1 Contaminant toxicity summaries .................................................. $5-1$

5.1 .1 Manganese ..................................................................... $5-1$

5.1 .2 Molybdenum .................................................................. $5-4$

5.1 .3 Uranium ......................................................................... 5-6

5.2 Contaminant interactions............................................................. 5-10

5.3 Contaminant risk factors ............................................................. 5-12

6.0 HUMAN HEALTH RISK EVALUATION............................................... $6-1$

6.1 Potential noncarcinogenic health effects ...................................... $6-1$ 
TABLE OF CONTENTS (Concluded)

Section

Page

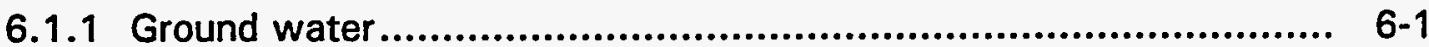

6.1.2 Surface water/sediment ...................................................... 6-4

6.2 Potential carcinogenic health effects............................................. $6-4$

6.3 Limitations of the human health risk evaluation................................... $6-6$

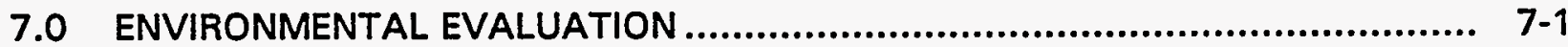

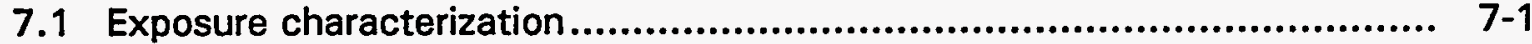

7.2 Ecological receptors ................................................................ $7-2$

7.2.1 Terrestrial resources ........................................................ $7-2$

7.2 .2 Aquatic organisms ......................................................... $7-4$

7.3 Contaminants of ecological concern ............................................ $7-5$

7.3.1 Ground water .................................................................. 7-5

7.3.2 Surface water/sediment ................................................... 7-6

7.4 Potential impacts to wildlife and plants ........................................... $7-6$

7.4.1 Terrestrial risk .................................................................. 7-6

7.4 .2 Aquatic risk ........................................................... 7-10

7.5 Limitations of the ecological risk assessment.................................. $7-11$

7.6 Summary .......................................................................... $7-12$

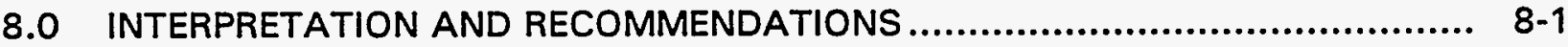

8.1 Risk summary ....................................................................... 8-1

8.2 Limitations of this risk assessment ............................................... $8-1$

8.3 Ground water criteria .................................................................. 8-3

8.4 Risk mitigation measures .......................................................... $8-5$

8.5 Recommendations ................................................................. 8-6

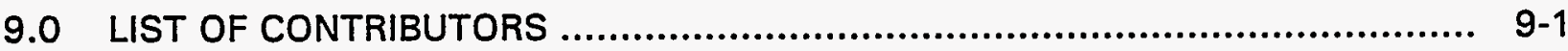

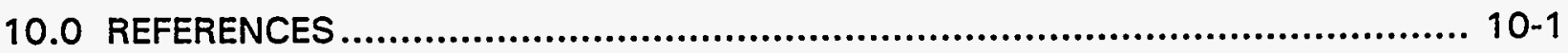




\section{LIST OF FIGURES}

Figure

Page

2.1 Canonsburg, Pennsylvania, site

2.2 Site map showing location of monitor wells. Canonsburg, Pennsylvania, site .....

2.3 Industrial park configuration, Canonsburg, Pennsylvania, site

2.4 Hydrogeologic cross section (A-A'), Canonsburg, Pennsylvania, site

2.5 Hydrogeologic cross section (B-B'), Canonsburg, Pennsylvania, site

2.6 Potentiometric surface map for the unconsolidated materials, Canonsburg, Pennsylvania, site

2.7 Potentiometric surface map for the shallow bedrock, Canonsburg, Pennsylvania, site

2.8 Existing land uses (1980), Canonsburg, Pennsylvania, site vicinity

2.9 Land use zoning, Canonsburg, Pennsylvania, site vicinity.

2.10 Domestic well locations, Canonsburg, Pennsylvania, site vicinity.

3.1 Trilinear plot showing anion-cation composition of background wells 410 and 504, and downgradient well 414, Canonsburg, Pennsylvania, site.

3.2 Ground water parameter versus time plots of wells $410,414,504$, and 509, Canonsburg, Pennsylvania, site

3.3 Molybdenum versus time plot for background wells 410 and 504, and downgradient wells 412, 413,414,505, 506, and 509, Canonsburg,

Pennsylvania, site

3.4 Uranium versus time plot for background wells 410 and 504 , and downgradient wells 412, 413, 414, 505, 506, and 509, Canonsburg, Pennsylvania, site

3.5 Surface water and sediment sample locations, Canonsburg, Pennsylvania, site

4.1 Conceptual model, Canonsburg, Pennsylvania, site

4.2 Probability distribution of manganese concentrations for the unconsolidated material, Canonsburg, Pennsylvania, site

4.3 Probability distribution of molybdenum concentrations for the unconsolidated material, Canonsburg, Pennsylvania, site

4.4 Probability distribution of uranium concentrations for the unconsolidated material, Canonsburg, Pennsylvania, site

4.5 Probability distributions for tap water ingestion rates .................................. 4-16

4.6 Probability distributions for body weight ................................................ 4-17

4.7 Probability distribution of manganese exposure to children based on ground water ingestion, Canonsburg, Pennsylvania, site........................................ 4-19

4.8 Probability distribution of molybdenum exposure to children based on ground water ingestion, Canonsburg, Pennsylvania, site

4.9 Probability distribution of uranium exposure to children based on ground water ingestion, Canonsburg, Pennsylvania, site.

4.10 Probability distribution of uranium exposure over a lifetime based on ground water ingestion, Canonsburg, Pennsylvania, site. 


\section{LIST OF FIGURES (Concluded)}

Figure

Page

5.1 Manganese toxicity ranges............................................................ $5-5$

5.2 Molybdenum toxicity ranges ............................................................ $5-7$

5.3 Half-lives and emissions from decay chain of uranium-238 ......................... $5-8$

5.4 Uranium toxicity ranges ................................................................ 5-11

6.1 Health effects of potential manganese exposure ranges to children, Canonsburg, Pennsylvania, site

6.2 Health effects of potential molybdenum exposure ranges to children, Canonsburg, Pennsylvania, site.

6.3 Health effects of potential uranium exposure ranges to children, Canonsburg, Pennsylvania, site

6.4 Excess lifetime cancer risk from potential uranium-234/238 exposure ranges, Canonsburg, Pennsylvania, site.

7.1 Location of riparian vegetation growing along Chartiers Creek, Canonsburg, Pennsylvania, site 


\section{LIST OF TABLES}

Table

CS.1 Hypothetical future human health effects from drinking contaminated ground water from the Canonsburg site

3.1 Monitor well and surface water sampling information, Canonsburg,

Pennsylvania, site

3.2 Comparison of background and on-site ground water quality data from the unconsolidated materials, Canonsburg, Pennsylvania, site (1986-1993, filtered water samples)

3.3 Contaminants of potential concern in ground water, Canonsburg, Pennsylvania, site

3.4 Stable species of constituents of concern in the ground water system at the Canonsburg, Pennsylvania, site

3.5 Comparison of upstream and downstream water quality data from Chartiers Creek, Canonsburg, Pennsylvania, site (1989-1993, unfiltered water samples)

3.6 Comparison of upstream and downstream sediment data from Chartiers Creek, Canonsburg, Pennsylvania, site (1993).

4.1 Exposure dose calculations and equation definitions for ground water ingestion and dermal contact for the future hypothetical adult scenario, Canonsburg, Pennsylvania, site.

4.2 Exposure dose calculations and equation definitions for incidental surface water ingestion and dermal contact by children in Chartiers Creek, Canonsburg, Pennsylvania, site.

4.3 Exposure dose calculations and equation definitions for incidental sediment ingestion by children in Chartiers Creek, Canonsburg, Pennsylvania, site....

4.4 Exposure dose calculations and equation definitions for ingestion of fish from Chartiers Creek by adults, Canonsburg, Pennsylvania, site

5.1 Toxicity values: potential noncarcinogenic effects

6.1 Estimated doses and toxicity values for the surface water and sediment exposure pathways in Chartiers Creek, Canonsburg, Pennsylvania, site.

6.2 Carcinogenic risk for the unconsolidated layer ground water ingestion pathway, Canonsburg, Pennsylvania, site

7.1 Occurrence of constituents detected in Chartiers Creek surface water, Canonsburg, Pennsylvania, site vicinity

7.2 Occurrence of constituents detected in Chartiers Creek sediment, Canonsburg, Pennsylvania, site vicinity.

7.3 Comparison of contaminants of potential concern in ground water with available water quality values, Canonsburg, Pennsylvania, site 


\section{LIST OF TABLES (Concluded)}

Table

Page

8.1 Concentration limits of constituents

8-4 


\section{LIST OF ACRONYMS}

Acronym

$A C L$

AEC

$B C F$

DOE

EPA

HEAST

IRIS

LD

LOAEL

MCL

MSL

NCP

NEPA

NOAEL

NRC

PEIS

Rfd

TAC

TDS

UMTRA

UMTRCA

\section{Definition}

alternate concentration limit

Atomic Energy Commission

bioconcentration factor

U.S. Department of Energy

U.S. Environmental Protection Agency

Health Effects Assessment Summary Tables

Integrated Risk Information System

lethal dose

lowest-observed-adverse-effect level

maximum concentration limit

mean sea level

National Contingency Plan

National Environmental Policy Act

no-observed-adverse-effect level

Nuclear Regulatory Commission

Programmatic Environmental Impact Statement

reference dose

technical assistance contractor

total dissolved solids

Uranium Mill Tailings Remedial Action

Uranium Mill Tailings Control Act 


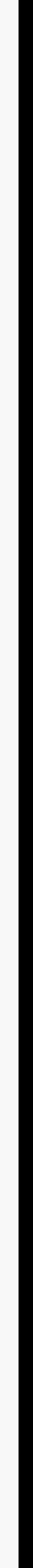




\subsection{INTRODUCTION}

The purpose of this baseline risk assessment is to determine whether ground water contamination at the Canonsburg, Pennsylvania, uranium processing site could adversely affect human health or the environment. The Canonsburg site is one of 24 abandoned uranium processing sites undergoing remediation in accordance with the requirements of the Uranium Mill Tailings Radiation Control Act (UMTRCA) of 1978 (42 USC $\$ 7901$ et seq.) under the oversight of the U.S. Department of Energy (DOE) Uranium Mill Tailings Remedial Action (UMTRA) Project.

Under the UMTRA Surface Project, the DOE has stabilized contaminated materials in a disposal cell that minimizes radon and other radiation emissions and further contamination of ground water. Remedial action at the Canonsburg site was completed in 1985 with stabilization of tailings, contaminated materials, and soils in a disposal cell at the former uranium processing site.

This risk assessment is a baseline assessment in the sense that it describes existing ground water conditions at the site. This document evaluates the potential for public health or environmental risks related to ground water contamination that may need attention before the site is fully characterized.

The risk assessment is based on available ground water data from wells and surface water data at the processing site. Major exposure pathways have been identified and thoroughly examined for this risk assessment.

This risk assessment follows the basic framework outlined by the U.S. Environmental Protection Agency (EPA) (1989a) for evaluating hazardous waste sites to assess potential health and environmental impacts. The risk assessment process consists of the following steps:

- Contamination characterization and data evaluation.

- Selecting chemical data for the risk assessment.

- Comparing sample results with background data.

- Selecting chemicals of potential concern.

- Discussing contaminant fate and transport.

- Exposure assessment.

- Characterizing exposure settings.

- Identifying exposure pathways.

- Identifying potentially exposed populations.

- Quantifying exposures. 
- Toxicity assessment.

- Identifying toxicity values.

- Evaluating noncarcinogenic effects of chemicals.

- Evaluating carcinogenic effects of radionuclides and chemical carcinogens.

- Public health risk characterization.

- Comparing toxicity ranges with predicted exposure ranges.

- Combining risks across exposure pathways and multiple contaminants.

- Characterizing noncarcinogenic and carcinogenic risks.

- Environmental risk.

- Characterizing potential biota exposure pathways.

- Identifying potential ecological receptors.

- Evaluating environmental risk qualitatively.

The framework is incorporated in the methodology developed to evaluate current human health risks at UMTRA Project sites and to estimate risks from potential future use of contaminated ground water or surface water near the former uranium processing site (DOE, 1994).

This risk assessment will support decisions made for the UMTRA Ground Water Project. The DOE was authorized to conduct ground water remediation under the UMTRCA Amendments Act (42 USC $\$ 7922$ et seq.) and will determine site-specific ground water compliance strategies for each site. This risk assessment provides information to assist in determining the site-specific ground water compliance strategy for the Canonsburg site. 


\subsection{SITE DESCRIPTION}

The Canonsburg site is in the southwestern corner of the borough of Canonsburg, Washington County, in southwestern Pennsylvania. The site lies approximately 20 miles (mi) (30 kilometers [km]) southwest of downtown Pittsburgh (Figure 2.1) in a mixed commercial/residential area and comprises approximately 30 acres (ac) (12 hectares [ha]). The Canonsburg site is bounded by Chartiers Creek to the west, north, and east and by Conrail railroad tracks to the south (Figure 2.2).

\subsection{SITE BACKGROUND}

In the early 1900s, the Standard Chemical Company developed a method to extract and concentrate radium from carnotite ore. The company established its radium processing facilities at Canonsburg, Pennsylvania, and produced the first marketable quantities of radium in 1913. The company ceased processing operations in the early 1920s (DOE, 1983a,b).

The Vitro Manufacturing Company purchased the Canonsburg facility in 1933 to extract uranium, vanadium, and radium from various ores, concentrates, and residues. From 1942 until the facility's closing in 1957, the operations focused on producing uranium concentrates. The only customer during this period was the U.S. Government. The uranium, and other rare metals, were extracted from both company-owned and government-owned ores, concentrates, and residues. Ores, concentrates, and scrap materials were brought from a number of Atomic Energy Commission (AEC) installations to the Canonsburg site for uranium recovery. All solid process wastes were stored temporarily on the Canonsburg site. The liquid process wastes were discharged into the former swamp in Area $C$, which subsequently drained into Chartiers Creek. Area $C$ has since been cleaned up to unrestricted use criteria in accordance with EPA regulations (MK-F, 1986). The area is backfilled with clean soil.

On 1 November 1953 the U.S. Government and the Vitro Corporation entered into a contract requiring Vitro to process certain government-owned materials. The contract required Vitro to store the residues from this operation until 1 November 1955, because the AEC believed the residues might contain recoverable uranium. After attempts by the AEC to identify commercial interest in the material, the uranium in the residue was deemed "unrecoverable" and the AEC approved the transfer of 11,600 tons $(10,500$ tonnes) of material to the Burrell site, which is about $50 \mathrm{mi}(80 \mathrm{~km})$ northeast of the Canonsburg site. This material contained approximately 6 tons (5.4 tonnes) of uranium oxide and was transported to the Burrell site during late 1956 and early 1957.

Recovery operations at the Vitro plant ceased by 1957. Vitro stored the remaining residues and processing wastes on the site. Vitro's final sourcematerial license expired, and in May 1961, Vitro applied to the AEC for another source-material license. On 21 June 1961 the AEC granted Vitro a license for 


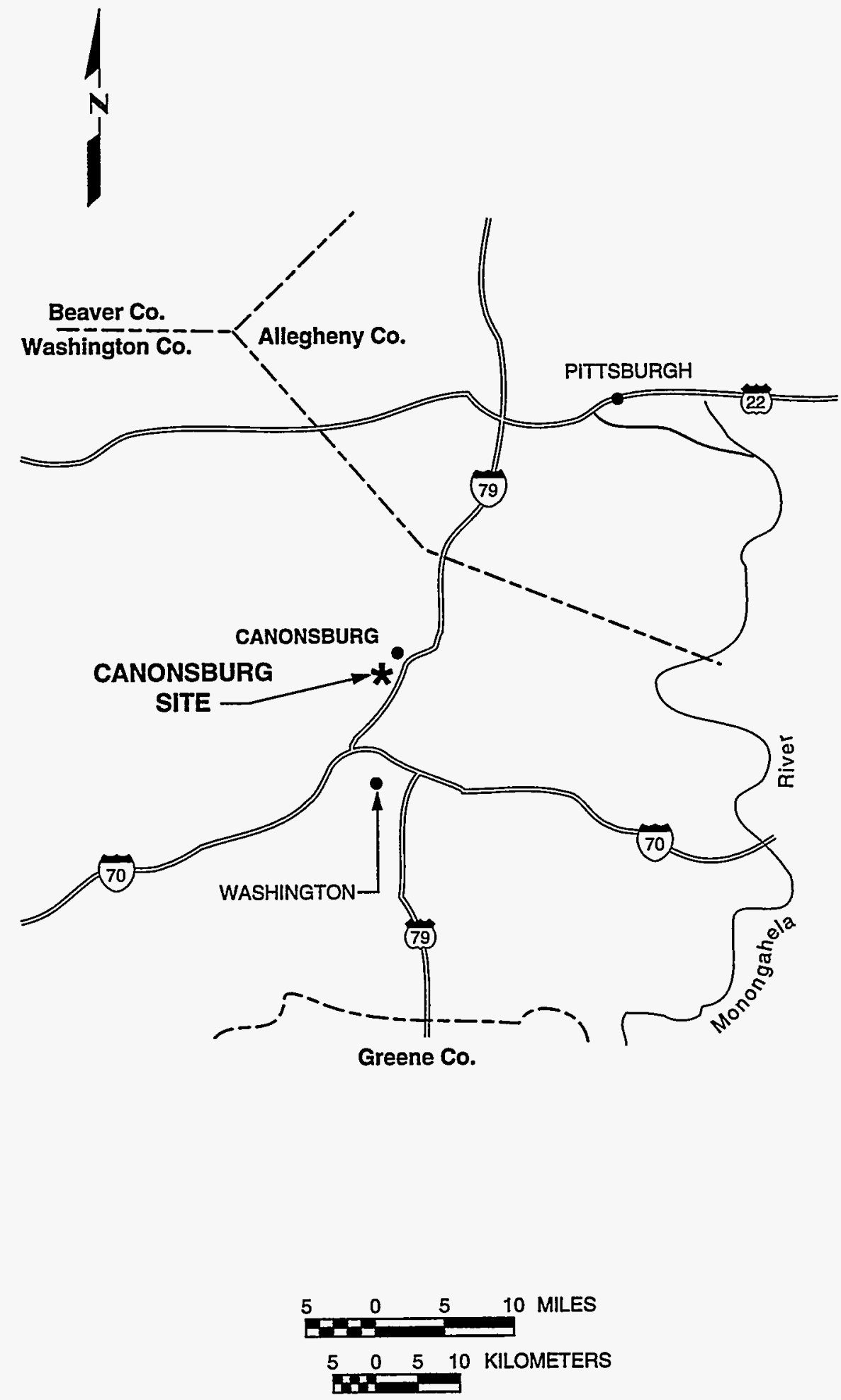

FIGURE 2.1

CANONSBURG, PENNSYLVANIA, SITE 


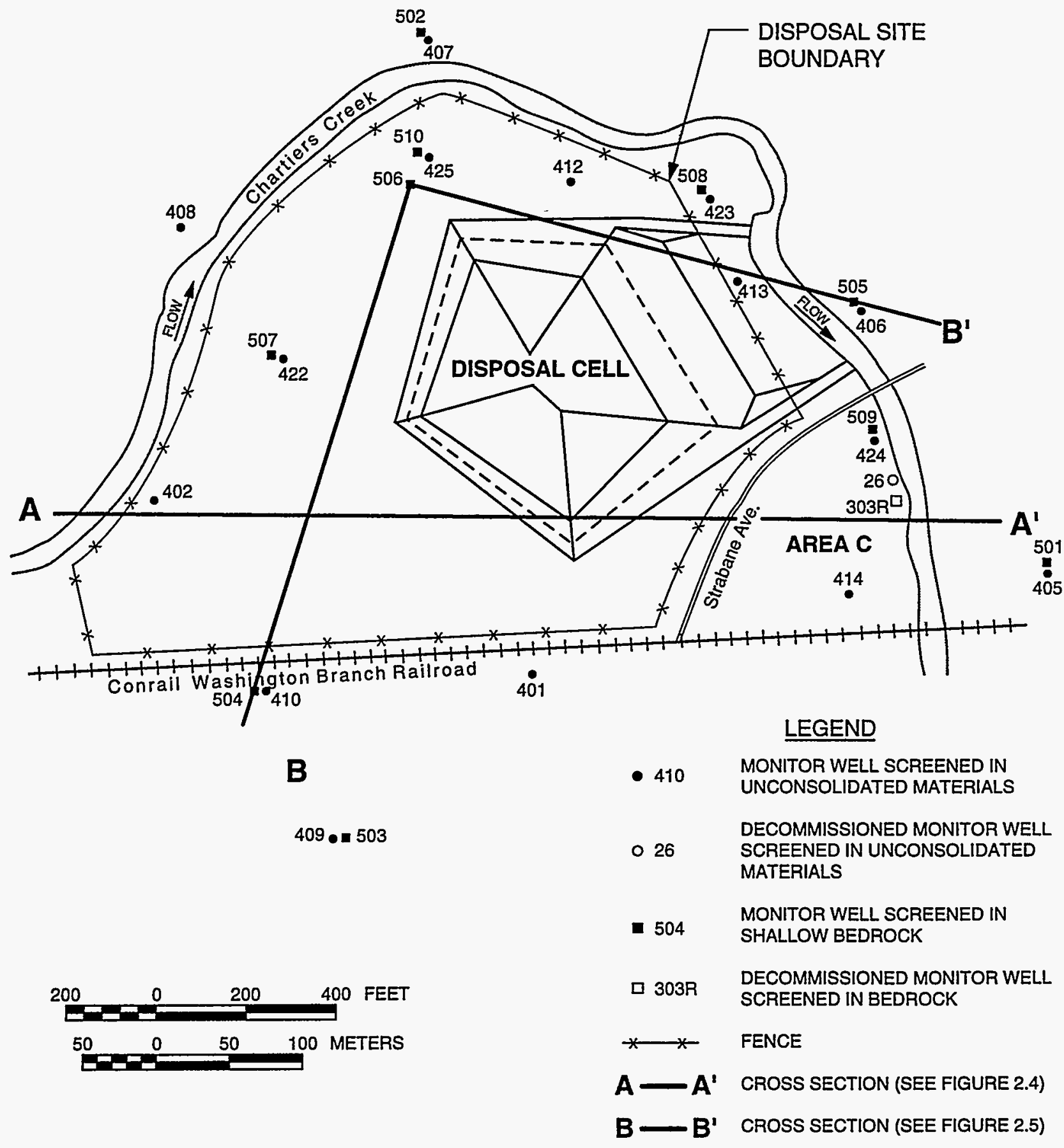

FIGURE 2.2

SITE MAP SHOWING LOCATION OF MONITOR WELLS

CANONSBURG, PENNSYLVANIA, SITE 
storage of up to 23 tons ( 21 tonnes) of uranium contained in approximately 4500 tons (4080 tonnes) of material.

The real property was sold to developers in 1962, while Vitro retained title to the uranium-containing materials. Before 1964 an effort was made to decontaminate the immediate plant area, and all contaminated material was moved to a main stockpile of uranium-bearing material located in Area A. In 1965 the commonwealth of Pennsylvania granted Vitro a permit to move this pile to Area $C$ where it was buried beneath a relatively impermeable layer of slag and covered by clean fill. Vitro's source-material license was then terminated and the Canonsburg site was developed into the Canon Industrial Park in 1966 (Figure 2.3).

On 8 November 1979 the DOE designated the Canonsburg site as eligible for remedial action under the UMTRCA. Effective 5 September 1980, the DOE and the commonwealth of Pennsylvania entered into a cooperative agreement (under the UMTRCA) that set forth the terms and conditions for a DOE and commonwealth cooperative remedial action effort (DOE, 1983a, 1983b). In 1982 the commonwealth of Pennsylvania acquired the Canonsburg site in accordance with the cooperative agreement between the state and the DOE (Licensing Implementation Plan, 1992).

Between 1984 and 1986 contaminated soils and materials (including those from Area $\mathrm{C}$ ) were stabilized in a permanent disposal cell (Figure 2.2). Approximately 172,000 cubic yards $\left(\mathrm{yd}^{3}\right)\left(132,000\right.$ cubic meters $\left.\left[\mathrm{m}^{3}\right]\right)$ of contaminated materials were stabilized in the disposal cell, which covers approximately 6 ac (2.4 ha) of the site (MK-Ferguson, 1986; Licensing Implementation Plan, 1992). The eastern toe of the cell was placed on fill material above the existing grade. The remainder of the site was evenly and smoothly graded to provide adequate site drainage and was revegetated with native grasses (DOE, 1983b). As shown in Figure 2.2, fencing was installed around the majority of the site, including Areas A and B. Area C, southeast of the designated disposal site, is not fenced and is being evaluated for public use.

The site is being monitored according to the Surveillance and Maintenance Plan for the Canonsburg site (DOE, 1992). The site will remain in the poststabilization, prelicensing status until the U.S. Nuclear Regulatory Commission (NRC) issues a license for long-term surveillance and maintenance under the provisions of 10 CFR Part 40. The general license becomes effective for the Canonsburg site after the NRC concurs with the completion of the surface remedial action and approves the long-term surveillance plan. Upon licensing, ownership of the site, with the exception of Area C, will transfer from the commonwealth of Pennsylvania to the U.S. Government under the oversight of the DOE. The state will retain ownership of Area $C$. 


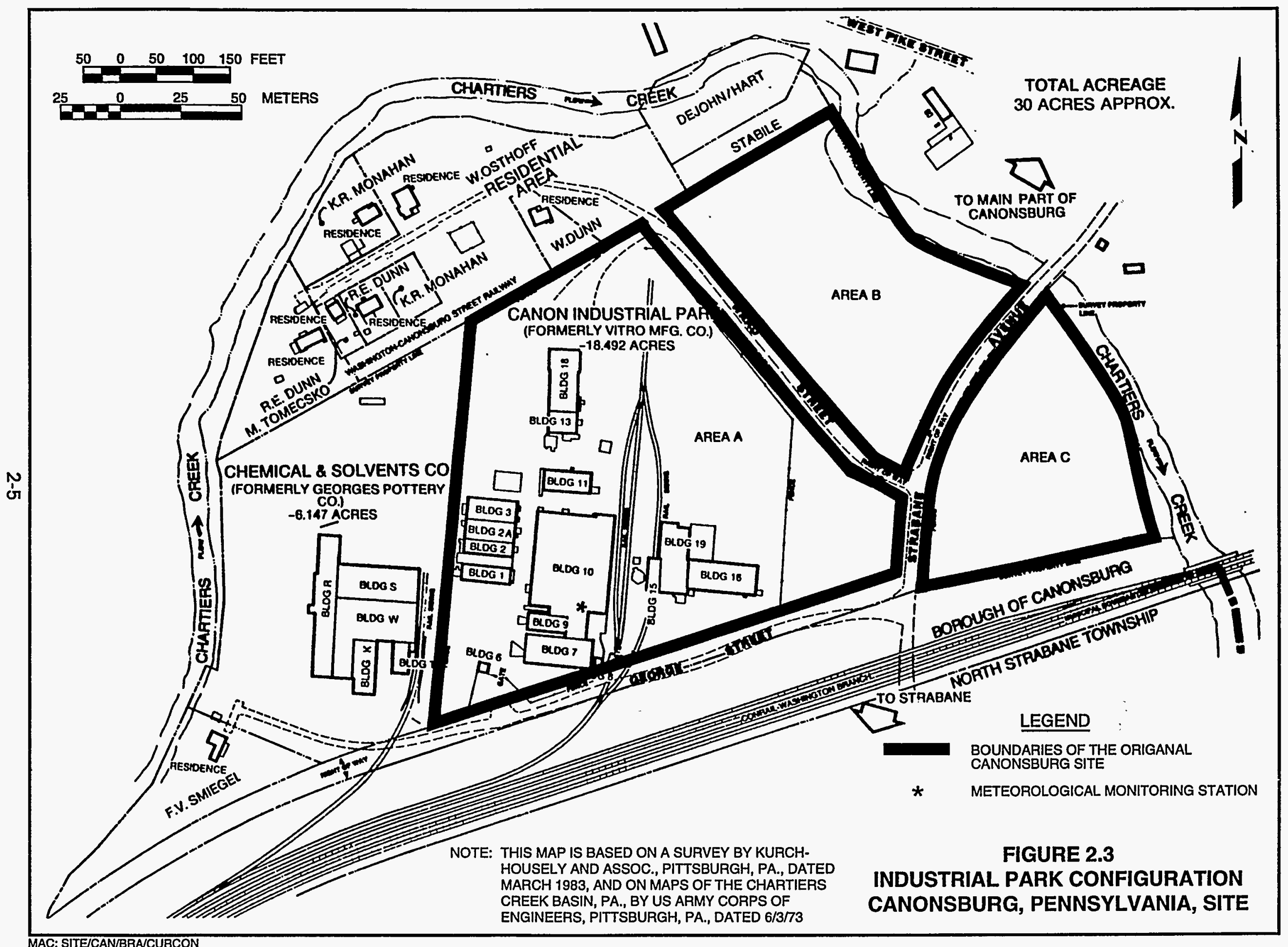


The Canonsburg site is in the humid continental climatic region. This region experiences distinct seasons with seasonal variations slightly moderated by the Great Lakes and the Atlantic seaboard (DOE, 1983a).

The average annual temperature at the Canonsburg site is $\mathbf{5 0}$ degrees Fahrenheit $\left({ }^{\circ} \mathrm{F}\right)$ (10 degrees Celsius $\left[{ }^{\circ} \mathrm{C}\right]$ ), based on data collected on the site from 1979 to 1981 . Based on these data, the winter temperatures at the site range from $-6^{\circ}$ to $63^{\circ} \mathrm{F}\left(-21^{\circ}\right.$ to $\left.17^{\circ} \mathrm{C}\right)$, with an average temperature of $28^{\circ} \mathrm{F}(-$ $\left.2^{\circ} \mathrm{C}\right)$; the summer temperatures range from $36^{\circ}$ to $95^{\circ} \mathrm{F}\left(2^{\circ}\right.$ to $\left.35^{\circ} \mathrm{C}\right)$, with an average temperature of $70^{\circ} \mathrm{F}\left(21^{\circ} \mathrm{C}\right)$. Generally mild summers are frequently humid due to invasions of tropical air from the Gulf of Mexico. The winter months are brisk with occasional periods of extreme cold. Spring and fall are transitional seasons, with moderate-to-cool temperatures. Rapid and wide variations in day-to-day weather conditions are common during the spring and fall (DOE, 1983a).

Based on data from the Pittsburgh International Airport from 1941 to 1980 , the average annual precipitation in the area is 38 inches. March and June are the wettest months, each averaging 3.8 inches, while February and November are the driest, each averaging 2.4 inches. The average annual snowfall in the site vicinity is 45 inches. The snow season typically occurs from October to May, with the heaviest snowfall in January (DOE, 1983a).

The Canonsburg site is situated in the east-to-west-oriented Chartiers Creek valley, which channels wind flows. As a result, the predominant wind direction is west-to-northwest. Cross-valley winds (north and south) are limited to periods of relatively high wind speeds typically occurring in the winter as northerly winds. The average annual wind speed as measured from 1979 to 1980 at the Canonsburg site was $4.7 \mathrm{mi}(7.6 \mathrm{~km}$ ) per hour (DOE, 1983a).

\subsection{PHYSIOGRAPHIC SETTING}

The Canonsburg site is in southwestern Pennsylvania where the geologic structural setting consists of subparallel folds with northeastern axis orientation. Landfilling and earth-moving activities have altered the topography of the Canonsburg site, originally a low-lying flood plain. The site lies in the Chartiers Creek basin along the creek's southern bank, approximately $15 \mathrm{mi}(24 \mathrm{~km})$ upstream from its confluence with the Ohio River.

\section{$2.4 \quad$ HYDROGEOLOGY}

\subsubsection{Geology}

The Canonsburg site is on a sequence of unconsolidated materials overlying bedrock of the Pennsylvanian Casselman Formation (Figures 2.4 and 2.5). 


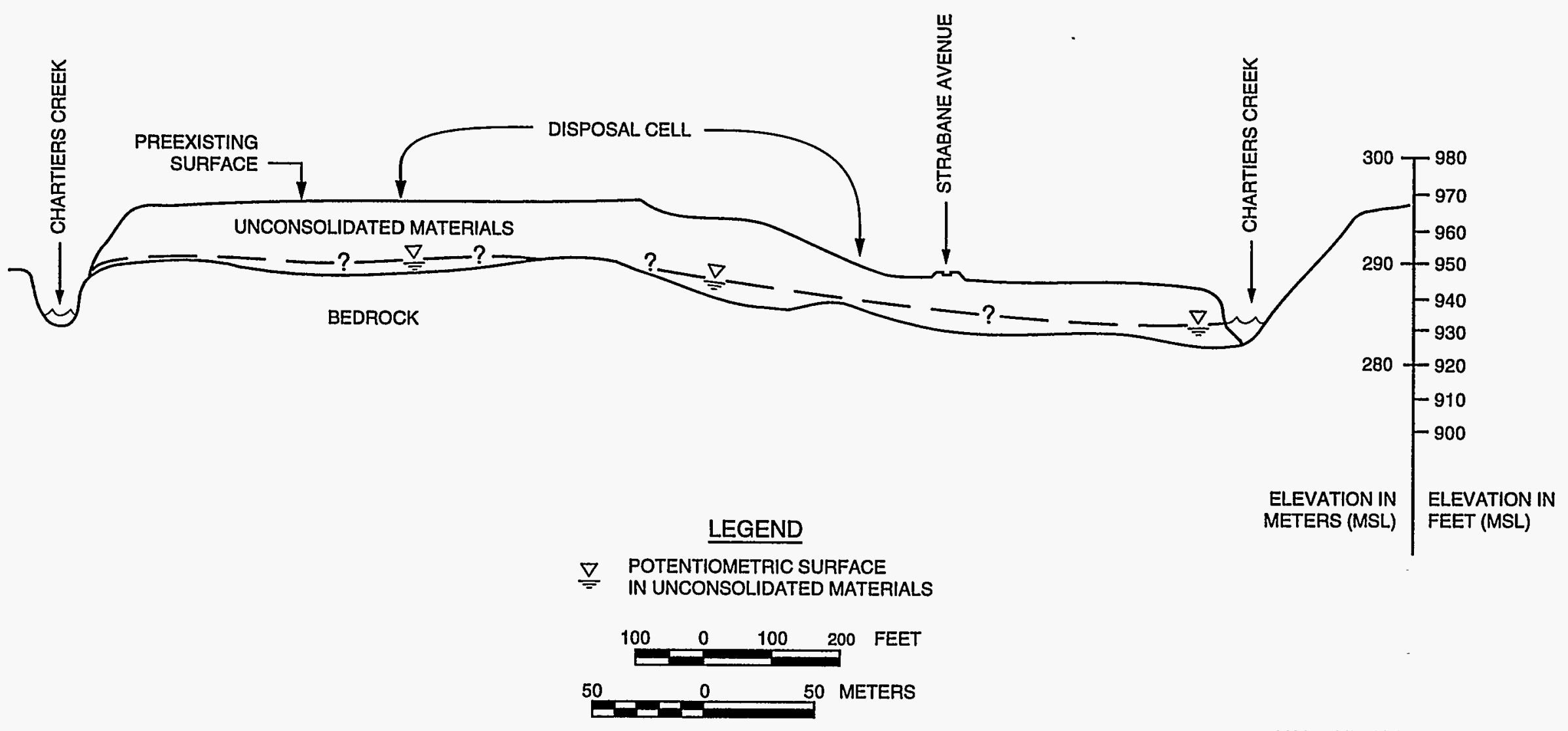

$M S L=$ MEAN SEA LEVEL

REDRAWN FROM DOE, 1983a

FIGURE 2.4

HYDROGEOLOGIC CROSS SECTION (A-A')

CANONSBURG, PENNSYLVANIA, SITE 
B

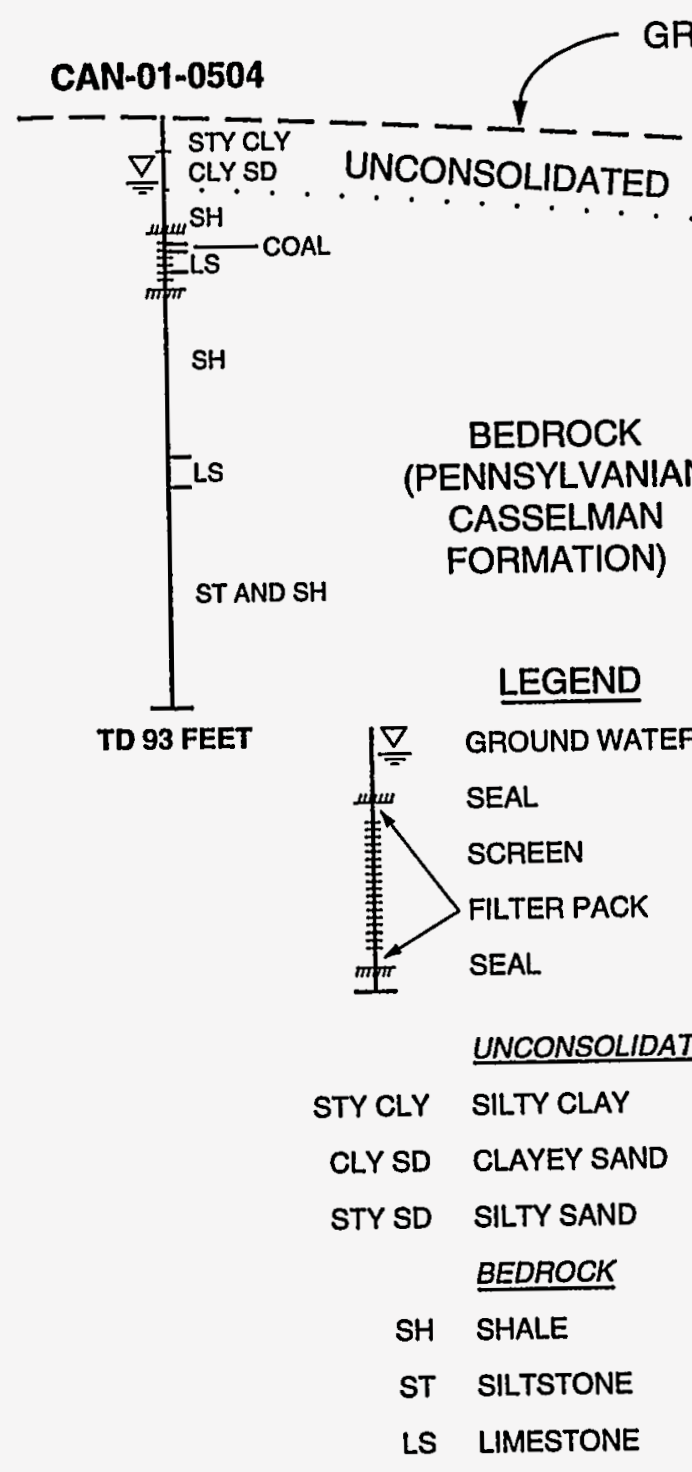

LS LIMESTONE
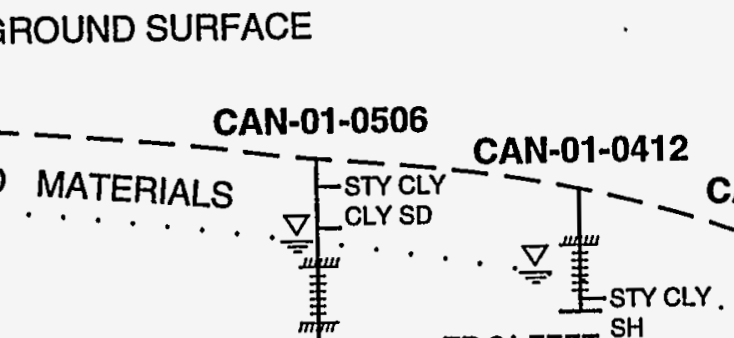

TD 90 FEET

TD 21 FEET ${ }^{\text {SH }}$

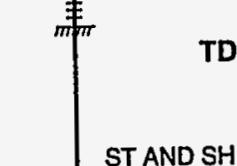

$\stackrel{N}{\infty}$
$\mathbf{B}^{\prime}$

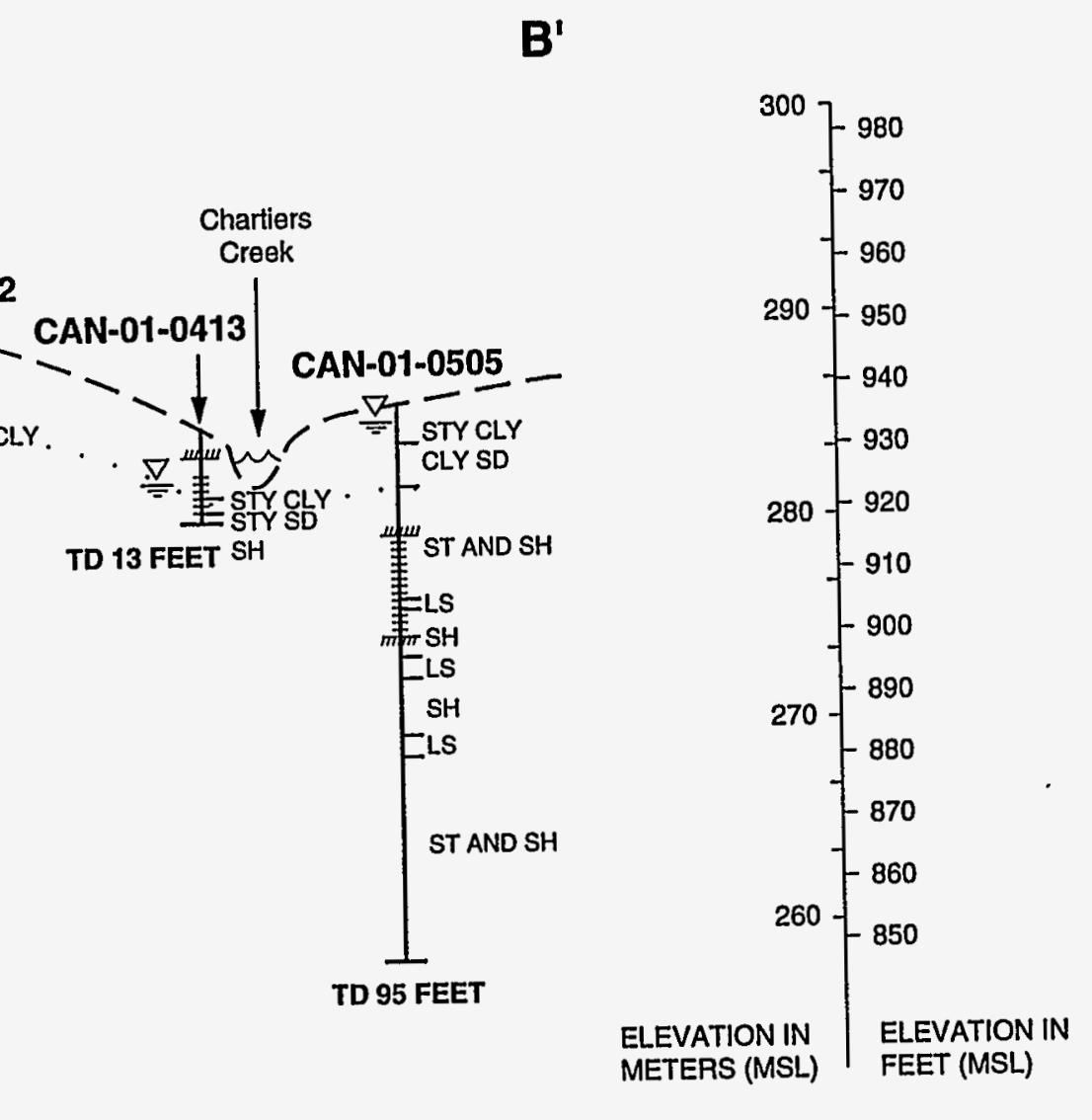

$M S L=$ MEAN SEA LEVEL

FIGURE 2.5

HYDROGEOLOGIC CROSS SECTION (B-B')

CANONSBURG, PENNSYLVANIA, SITE 


\section{Unconsolidated materials}

The unconsolidated materials generally consist of up to 30 feet $(\mathrm{ft}$ ) ( 9 meters [m]) of soil (sandy loam to silty clay loam), clay, alluvium, and fill material (cinders mixed with soil, stones/cobbles, and building rubble). The unconsolidated materials are heterogeneous beneath the site and do not form discrete, continuous units. Also, the permeability is variable due to the types and placement of the materials.

\section{Bedrock}

The lithology of the bedrock to a depth of $95 \mathrm{ft}(29 \mathrm{~m})$, based on lithologic logs of monitor wells CAN-01-0504 through -0506, consists predominantly of gray siltstone and shale, with some interbedded limestone, and sparse coal seams (Figure 2.5). Shale near the bedrock surface is broken and weathered to thin, brittle plates. The bedrock surface generally dips northeast at less than 1 degree. Fracturing was observed in core samples in the upper 5 to $20 \mathrm{ft}$ ( 2 to $6 \mathrm{~m}$ ) of the bedrock beneath the site. The interval of interest for evaluating potential bedrock contamination is the upper $25 \mathrm{ft}(7 \mathrm{~m})$ beneath the contact with the unconsolidated material, referred to as the "shallow bedrock." The shallow bedrock has been observed at the surface in the site vicinity.

\subsubsection{Hydrology}

Ground water is present in two distinct units beneath the site: the unconsolidated materials and the bedrock. Hydraulic interconnection appears between the two lithologic units with a generally downward vertical gradient from the unconsolidated materials to the shallow bedrock. The dominant boundary condition for ground water movement in the unconsolidated materials and shallow bedrock is Chartiers Creek. This creek surrounds the site on the west, north, and east and is the normal discharge zone for the water table and shallow bedrock ground water systems. Although Chartiers Creek is principally a gaining stream, minor gradient reversal in ground water flow may occur near the creek during periods of maximum flow. However, this should not substantially impact potential contaminant migration.

Although ground water is present in the unconsolidated materials and shallow bedrock beneath the site, neither unit is considered as an aquifer. This is primarily because the materials are not ideal for aquifer formation, the source of recharge to the shallow units is limited, and sustained yield to a well from these units is probably lacking.

\section{Unconsolidated materials}

Ground water occurs in the unconsolidated materials under unconfined (water table) conditions. Depth to ground water in the unconsolidated materials ranges from 3 to $14 \mathrm{ft}(0.9$ to $4.3 \mathrm{~m})$ beneath the ground surface. Figure 2.6 shows the configuration of the potentiometric surface and the approximate direction of 


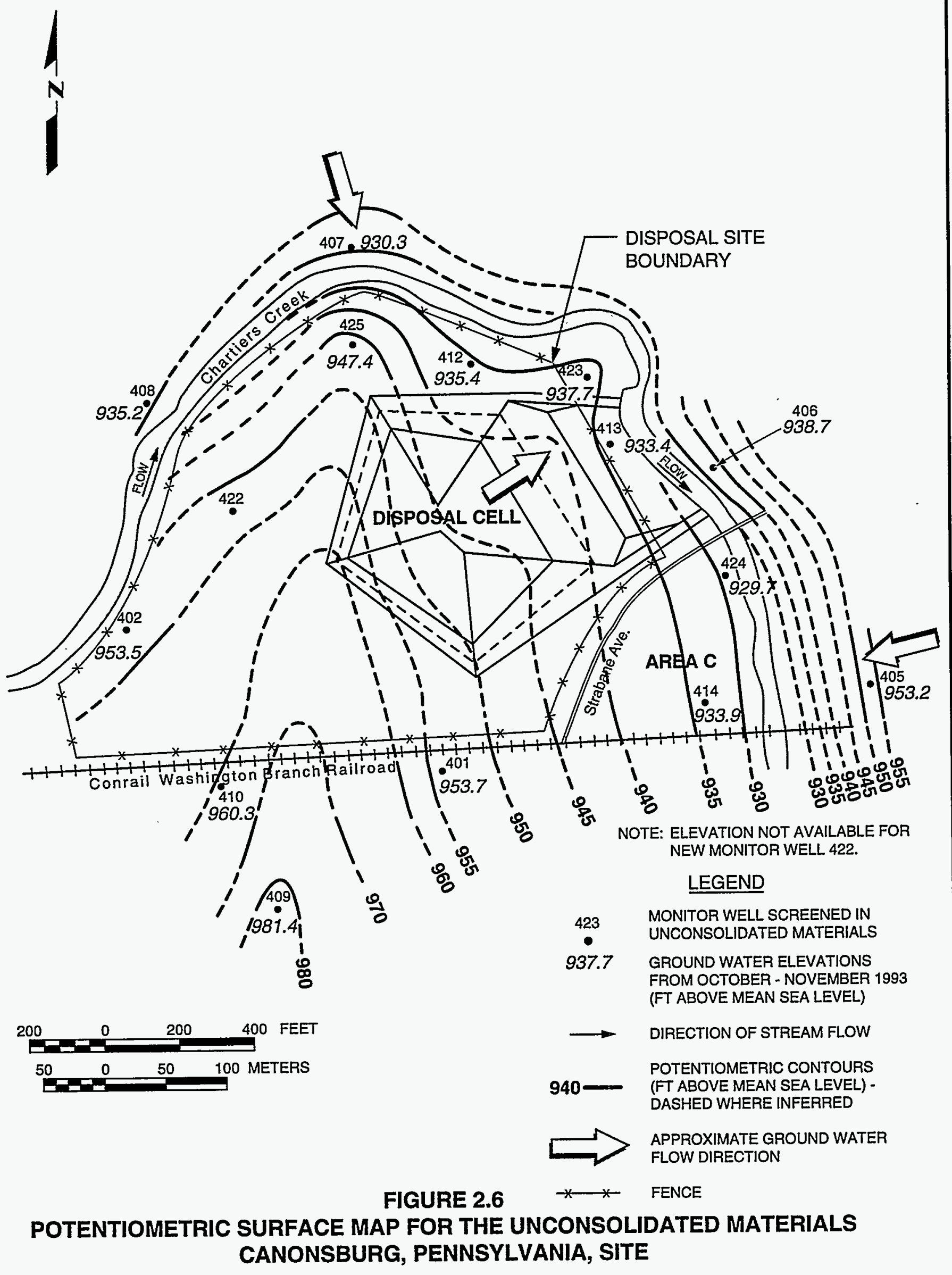


ground water flow in this unit; because of discontinuities and heterogeneities of the materials, the degree of hydraulic interconnection between areas beneath the site and the exact flow direction may be variable. Ground water in the unconsolidated materials is a result of precipitation infiltrating the unit with downward migration toward contact with the shallow bedrock. Water may perch on clay layers within the unit or on the shallow bedrock. Lateral continuity of ground water in this unit has not been definitively determined. Transmissivity, based on aquifer pumping tests in well 26, averaged 300 square feet $\left(\mathrm{ft}^{2}\right)$ per day (3.22 square centimeters $\left[\mathrm{cm}^{2}\right]$ per second) in the unconsolidated materials (DOE, 1983a), which, along with the estimated saturated thickness of $10 \mathrm{ft}(3 \mathrm{~m})$, a porosity of 0.2 , and a gradient of 0.027 , results in an approximate ground water velocity of $4 \mathrm{ft}$ per day $11.4 \times 10^{-3} \mathrm{~cm}$ per second).

\section{Bedrock}

Ground water occurs in the underlying shallow bedrock under semiconfined conditions. Figure 2.7 shows the potentiometric surface configuration and the approximate ground water flow direction in the shallow bedrock unit; because of discontinuities and the accumulation of ground water in fractured and weathered zones, the degree of hydraulic interconnection between areas beneath the site and the exact flow direction may be variable. Ground water in the shallow bedrock results from water infiltration from above into zones of secondary porosity where the shale bedrock is weathered or fractured. Some ground water appears to be present in deeper zones in the bedrock associated with limestone or more porous zones. This ground water is probably not related to surface infiltration due to intervening shale layers acting as aquitards, but is a result of ground water underflow. Transmissivity, based on aquifer pumping tests in well $303 R$, ranged from 0.167 to $3.94 \mathrm{~cm}^{2}$ per second in the shallow bedrock (DOE, 1983a), which, along with an estimated saturated thickness of $20 \mathrm{ft}(6 \mathrm{~m})$, a porosity of 0.2 , and a gradient of 0.016 results in an approximate ground water velocity range of 0.1 to $3 \mathrm{ft}$ per day $\left(4.3 \times 10^{-5}\right.$ to $1.0 \times 10^{-3} \mathrm{~cm}$ per second).

\subsection{SURFACE WATER}

The Canonsburg site lies along the southern bank of Chartiers Creek (Figure 2.2), a meandering stream 75 to $100 \mathrm{ft}(23$ to $30 \mathrm{~m})$ wide and about 10 $\mathrm{ft}(3 \mathrm{~m})$ deep. Chartiers Creek drains an area of approximately 80 square miles $\left(\mathrm{mi}^{2}\right)$ (200 square kilometers $\left[\mathrm{km}^{2}\right]$ ) upstream from the site and drains into the Ohio River $15 \mathrm{mi}(24 \mathrm{~km})$ downstream from the site. The average flow of Chartiers Creek past the site is 90 to $130 \mathrm{ft}^{3}$ per second 12.5 to $3.7 \mathrm{~m}^{3}$ per second). As discussed in Section 2.4.2, ground water in the unconsolidated material discharges into Chartiers Creek. 


\section{I \\ j}

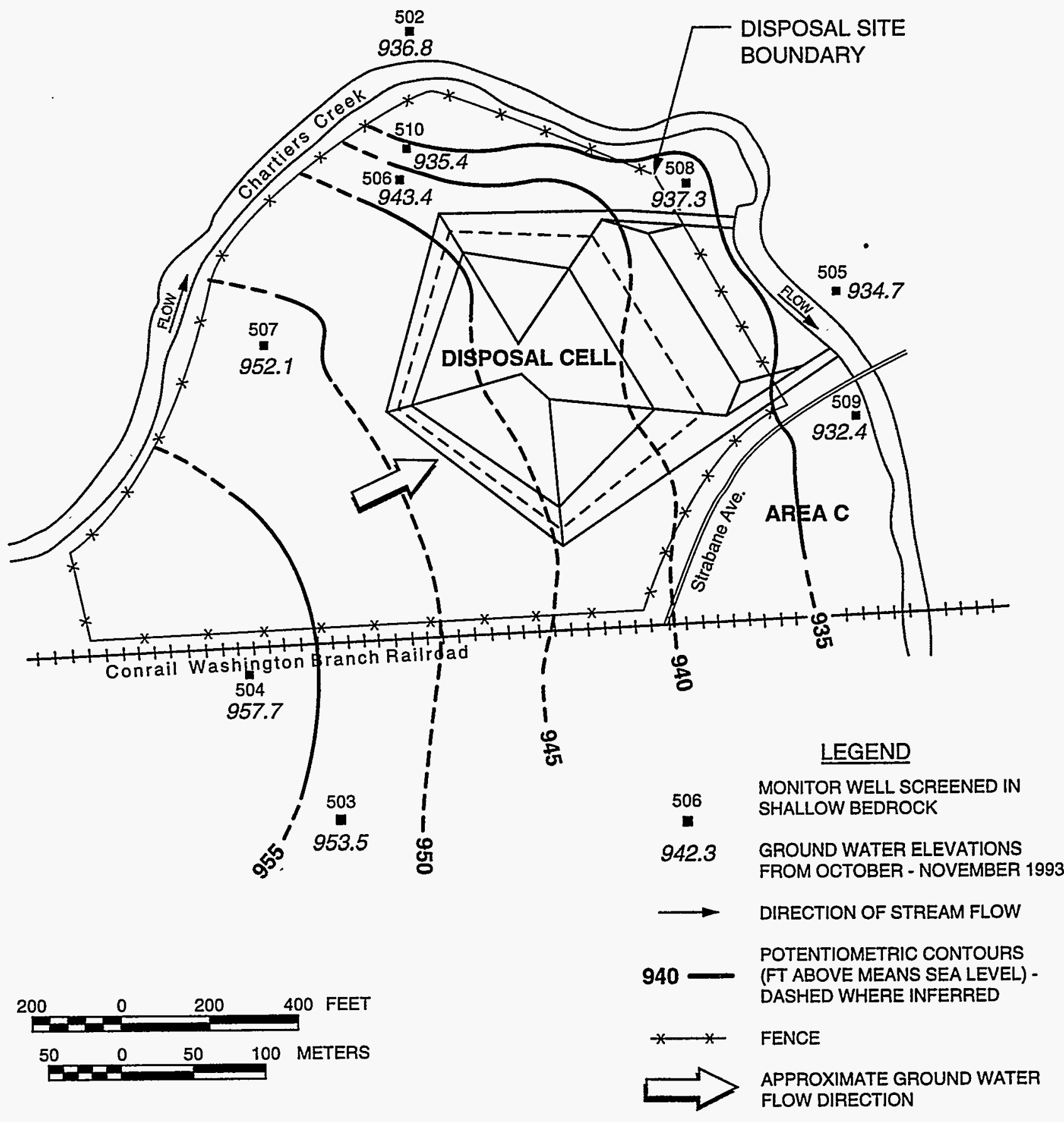

FIGURE 2.7

POTENTIOMETRIC SURFACE MAP FOR THE SHALLOW BEDROCK CANONSBURG, PENNSYLVANIA, SITE 


\subsection{LAND USE}

The former uranium processing site at Canonsburg, Pennsylvania is currently owned by the commonwealth of Pennsylvania. The site consists of the disposal site area and Area C (Figure 2.2). As discussed in Section 2.1, the disposal site area is within the zone where land use has been restricted. Upon the site licensing, ownership of the disposal site area will transfer from the commonweath of Pennsylvania to the U.S. Government under the oversight of the DOE. The state will retain ownership of the remaining portion of the Canonsburg site, Area C. That part of the site (Area $C$ ) is being considered for future public use.

A $1-\mathrm{mi}(1.6-\mathrm{km})$ radius around the site encompasses four municipalities: the borough of Canonsburg, the borough of Houston, Chartiers Township, and North Strabane Township. The borough of Houston and North Strabane Township are hydrologically upgradient or crossgradient of the site. The two other municipalities, the borough of Canonsburg and Chartiers Township, are on the opposite side of Chartiers Creek relative to the site. Ground water from their location flows toward the site and discharges into the creek. Therefore, these municipalities are not expected to be influenced by ground water flow from the site.

The primary land use in the site vicinity is residential. The closest residences are approximately 80 to $100 \mathrm{ft}(24$ to $30 \mathrm{~m})$ east of the site along Strabane Avenue and adjacent streets in the borough of Canonsburg. Residences are also directly south of the site in North Strabane Township on Latimer Avenue and west and southwest of the site in the borough of Houston.

North of the site along West Pike Street is a commercial and light industrial area. Businesses in this area include a gas station, car wash, car repair shop, car dealerships, woodcrafting company, and a heating and cooling company. Residences are north of this commercial/industrial area on Pike Street. A light industrial area is also located southwest of the site. Undeveloped areas are primarily northwest of the site in Chartiers Township and south of the site in North Strabane Township. Figure 2.8 shows the land uses within a 1-mile $(1.6-\mathrm{km})$ site radius. This map, taken from the Final Environmental Impact Statement for the Canonsburg site (DOE, 1983a), was based on information obtained from maps and local surveys. Recent site visits in 1994 indicate that land uses have changed only slightly with the possible addition of some new residential and commercial areas. Figure 2.9 shows the current land use zoning within this same area.

Based on 1990 data (Washington County Planning Commission, 1993), the populations of the municipalities in the site vicinity are as follows:

- Borough of Canonsburg - 9200

- Borough of Houston - 1445 


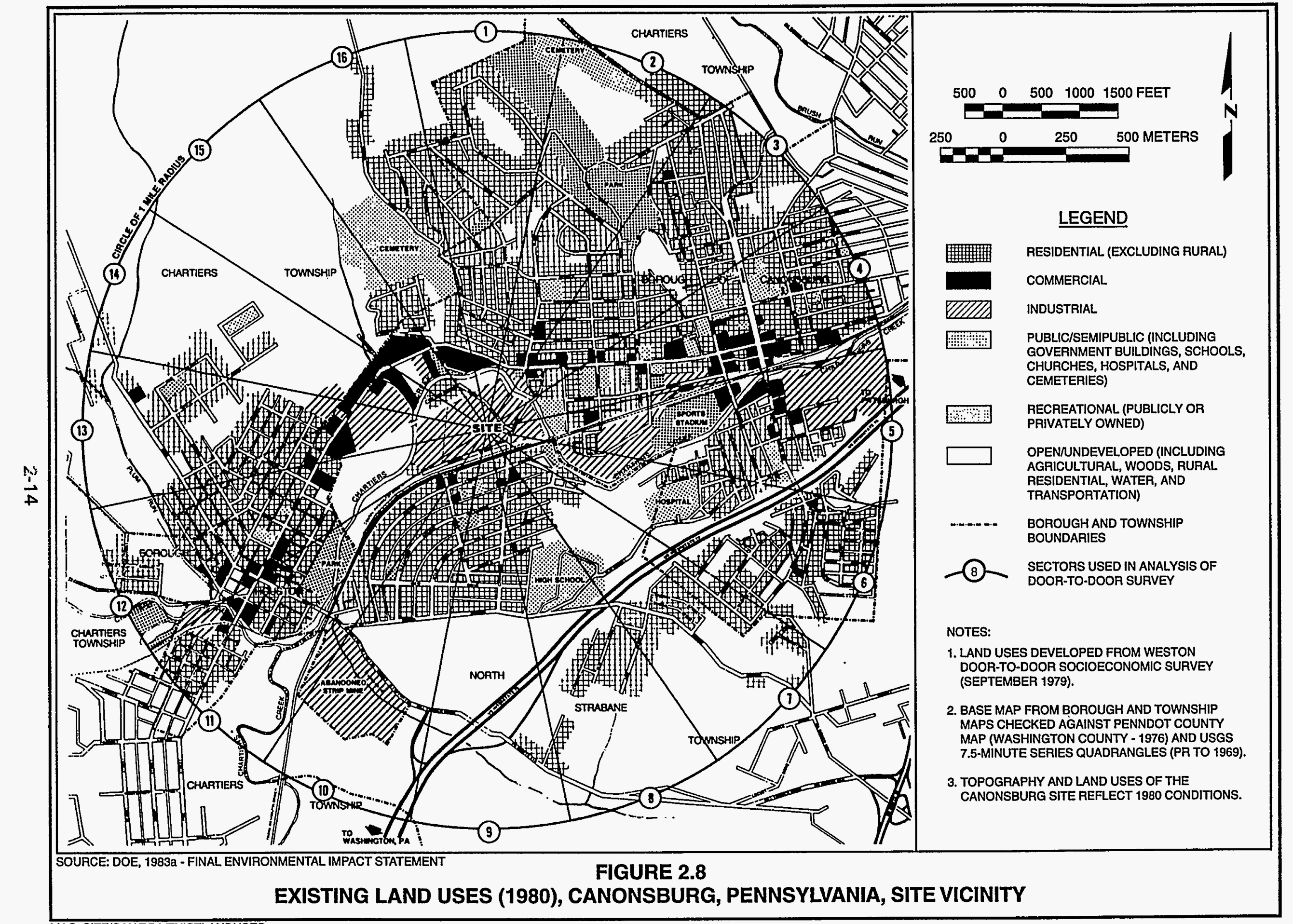




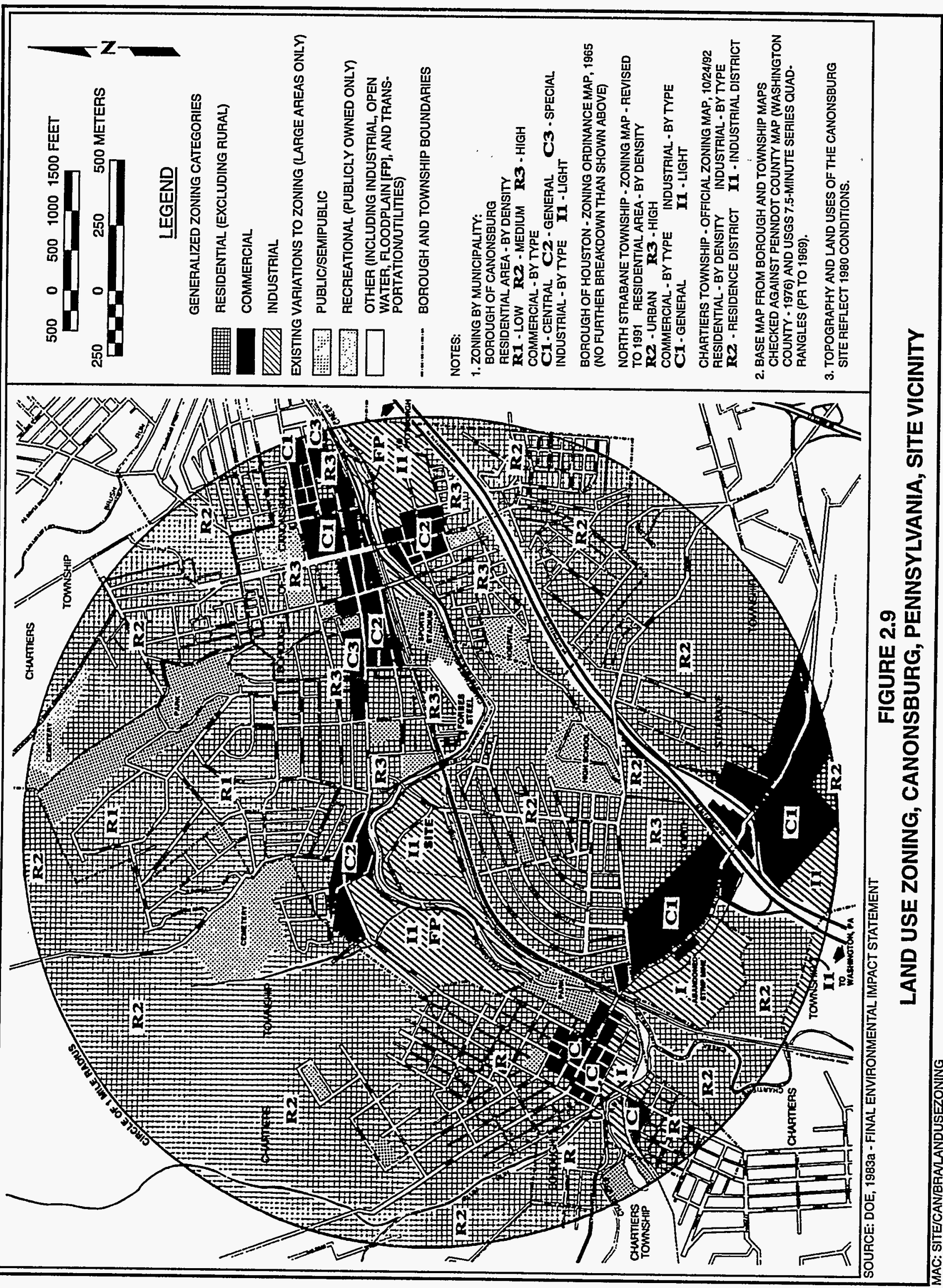


- Chartiers Township - 7603

- North Strabane Township - 8157

The population of Washington County has fluctuated but has remained at more than 200,000 for the past few decades. Based on data from 1990, the population for the county is 204,584 (Washington County Planning Commission, 1993).

The 1992-1993 Industrial Directory for Washington County lists 491 industries and businesses that employ 19,980 persons. Washington County's resident civilian labor force was approximately 95,400 in 1991, with approximately 66,860 persons working in the county. By categories, the major employers in the county are steel (1989 employees), electronics (1789 employees), mining (1276 employees), and plastics (1212 employees) (Washington County Board of Commissioners, 1993). Manufacturing in Washington County has declined over the years, primarily in steel and glass and their supporting industries. However, employment has increased in the government, wholesale/retail trade, and service industries. The major manufacturing employer in Canonsburg is Cooper Power Systems that employs approximately 1080 persons (Washington County Board of Commissioners, 1993).

Approximately 1590 farms are in Washington County, with an average size of 137 ac (55 ha). The main products are milk and dairy (Washington County Board of Commissioners, 1993).

\subsection{WATER USE}

Most residents of Canonsburg, Houston, North Strabane, and Chartiers, in the site vicinity, are connected to a municipal water supply system operated by the Pennsylvania-American Water Company. The Monongahela River, located east of the site, supplies the water for the system. There are two water intake stations: in Aldridge and in Pittsburgh, Pennsylvania. The Aldridge plant can treat about 60 million gallons (gal) (230 million liters [L]) of water per day, and the Pittsburgh plant can treat 80 million gal ( 300 million $L$ ) of water per day (Taylor, 1994). Washington District residents use approximately 10 million gal (38 million $L$ ) of water daily.

In April 1994 a water use survey was conducted within a $1-\mathrm{mi}(1.6-\mathrm{km})$ radius of the Canonsburg site. The purpose of the survey was to verify locations and status of domestic well information listed in previous surveys (DOE, 1983a), either provided by the Pennsylvania Geologic Survey (PGS, 1994) or obtained from personal communications with local residents. From this water use survey, approximately 16 wells were identified within a $1-\mathrm{mi}(1.6-\mathrm{km})$ site radius. Of these wells, 1 was in use, 11 were not in use, and 4 were abandoned.

Figure 2.10 shows the locations of the 12 wells that were not abandoned. Seven of these wells are upgradient of the site and would not be affected by any potentially migrating site-related contaminants. The remaining five wells are on the opposite side of Chartiers Creek relative to the site. Of these five 


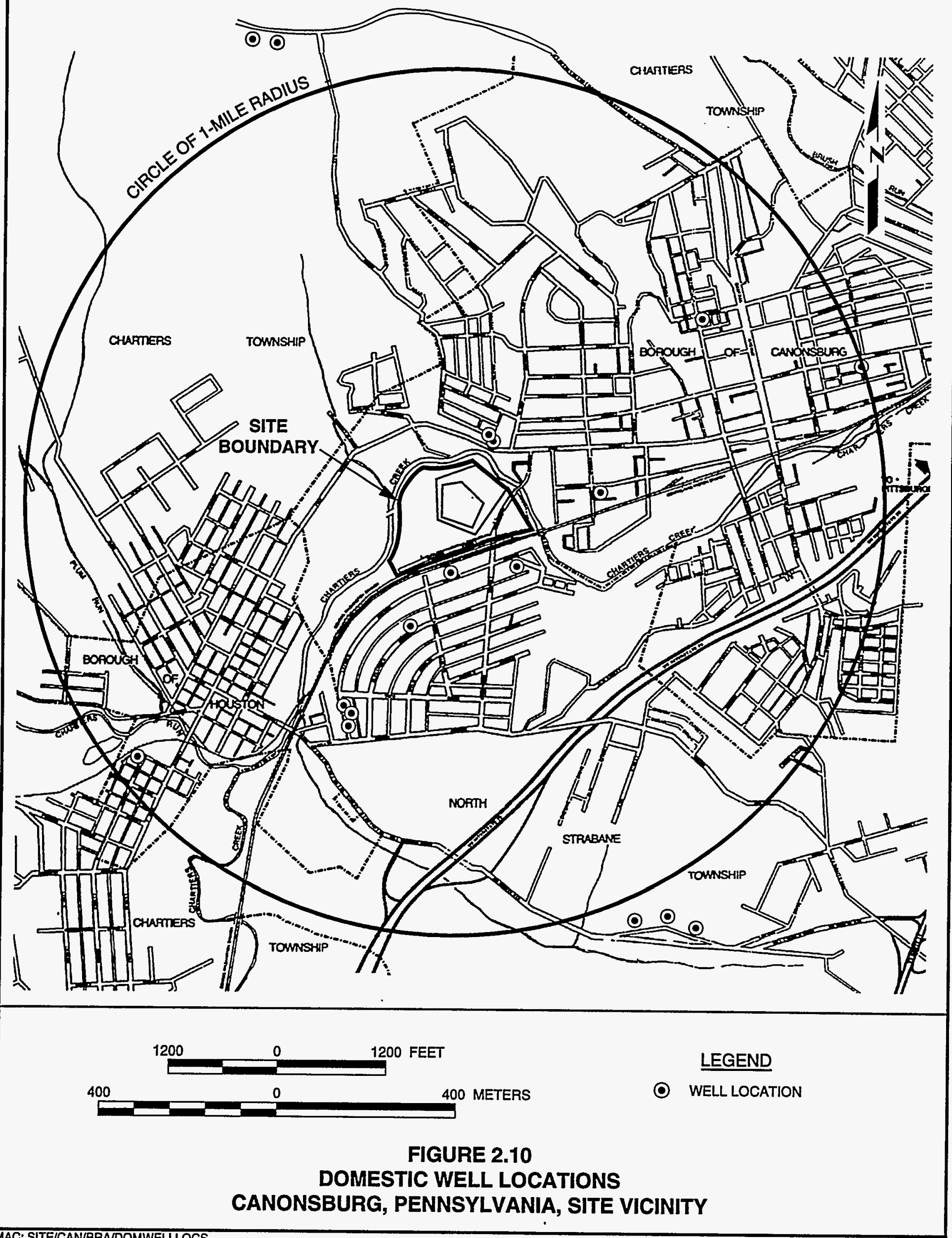


wells, only one is in use. This well is approximately $400 \mathrm{ft}(122 \mathrm{~m})$ north of the site and is used for washing cars, mixing cement, and watering the garden. Due to the location of this well it is not expected to be influenced by any ground water flow from the site. The ground water from this location flows toward the site and discharges into Chartiers Creek (Figure 2.6).

Figure 2.10 also shows the locations of five wells situated immediately outside the $1-\mathrm{mi}(1.6-\mathrm{km})$ radius. These wells are used for drinking as well as for all other domestic purposes. Three of these wells are just over $1 \mathrm{mi}(1.6 \mathrm{~km})$ south of the site. The municipal water supply does not service houses further south of this area; thus, individuals residing in this area most likely use well water. These wells are upgradient of the site and thus would not be affected by any potentially migrating contamination from the site. An additional two wells are approximately $1.3 \mathrm{mi}(2.1 \mathrm{~km})$ north of the site. Houses further north of this area also are not serviced by the municipal water supply system, and these residents also most likely use well water. Due to the distance from the site and the fact that these wells are located crossgradient (rather than downgradient) of the site, any ground water contamination potentially migrating from the site is not expected to affect ground water in this area.

Chartiers Creek, which flows along the western, eastern, and northern boundaries of the site, is designated by the commonwealth of Pennsylvania for the maintenance and propagation of fish species and protection of additional flora and fauna indigenous to a warm water habitat (PADER, 1992). The creek is not a potable water resource due to its ambient contamination upstream of the site (see Section 3.5 for detailed discussion). Local residents use the creek for fishing, swimming, and wading. Children have also been observed playing in the creek and on its banks. The types of fish found in the creek include carp, catfish, and bluegill (Templeton, 1993). 


\subsection{EXTENT OF CONTAMINATION}

Figure 2.2 is a map of the Canonsburg processing/disposal site showing the locations of the disposal cell, the DOE monitor well network, and other features mentioned in this discussion. Ground water is found at the site in unconsolidated near-surface materials and in the underlying bedrock. Filtered water quality data from 22 monitor wells were used to characterize background ground water and the nature and extent of ground water contamination at the site (DOE, 1995). Unfiltered water quality data are available for one sampling round (October 1993) for a limited number of constituents. For water samples from those wells used in this risk assessment, these data are generally consistent with the filtered water samples data (DOE, 1995). Therefore, unfiltered ground water data were not used for this risk assessment. However, for samples from other wells, some parameters indicated higher concentrations in unfiltered than in filtered water samples. Water quality data for unfiltered samples from six surface water locations were used to evaluate surface water quality (DOE, 1995). This risk assessment evaluates inorganic contamination at the Canonsburg site. Water samples were collected following applicable standard operating procedures in the Albuquerque Operations Manual (JEG, n.d.). Table 3.1 provides monitor well and surface water sampling site information, including the formations of completion and locations of monitor wells, years sampled, sampling rounds, and well depths.

\subsection{BACKGROUND GROUND WATER QUALITY DATA SUMMARY}

Background ground water quality is defined as ground water quality that would exist at this site if uranium processing had not occurred. Statistical and geochemical analyses have evaluated background ground water quality at the Canonsburg site.

Monitor wells 401,409 , and 410, completed in the near-surface, unconsolidated materiais, were evaluated as potential background wells. Monitor well 410 was most appropriate for estimating background levels in the unconsolidated material because it was completed in fill material that is characteristic of the site. Monitor well 409, on the other hand, is located off of the site and completed in unconsolidated alluvium and soils with different characteristics. Monitor well 401 was not used because, while the chemistry of ground water in well 401 is basically similar to that in well 410 , the concentrations of the radionuclides thorium-230 and polonium-210 are slightly higher than in well 410 . Because no historical ground water quality data exist for well 401, the possibility of site-related contamination could not be ruled out. Therefore, monitor well 401 was not included in the determination of background values.

Monitor wells 503 and 504 were considered potential background wells for the bedrock unit. Monitor well 503 is a deep well (112.9 ft [34.4 m]) screened in a limestone unit and, thus, is not representative of ground water conditions on the 
Table 3.1 Monitor well and surface water sampling information, Canonsburg, Pennsylvania, site

\begin{tabular}{|c|c|c|c|c|}
\hline Well numbe & Location & $\begin{array}{l}\text { Years } \\
\text { sampled }\end{array}$ & $\begin{array}{l}\text { Number } \\
\text { of rounds }\end{array}$ & $\begin{array}{l}\text { Screen } \\
\text { interval } \\
\text { (ft) }\end{array}$ \\
\hline \multicolumn{5}{|c|}{ Unconsolidated materials } \\
\hline \multicolumn{5}{|c|}{ Background wells } \\
\hline $\begin{array}{l}401 \\
409 \\
410\end{array}$ & $\begin{array}{l}\text { south of disposal cell } \\
\text { southwest of disposal cell } \\
\text { southwest of disposal cell }\end{array}$ & $\begin{array}{l}1986,1993 \\
1993 \\
1986-1993\end{array}$ & $\begin{array}{r}2 \\
1 \\
16\end{array}$ & $\begin{array}{l}7.5-12.2 \\
18.3(a) \\
11.4-16.0\end{array}$ \\
\hline \multicolumn{5}{|c|}{ Downgradient wells } \\
\hline $\begin{array}{l}405 \\
406 \\
407 \\
408 \\
412 \\
413 \\
414 \\
422 \\
423 \\
424 \\
425\end{array}$ & $\begin{array}{l}\text { across river from disposal cell } \\
\text { across river from disposal cell } \\
\text { across river from disposal cell } \\
\text { across river from disposal cell } \\
\text { north of disposal cell } \\
\text { northeast of disposal cell } \\
\text { southeast of disposal cell (Area C) } \\
\text { east of disposal cell } \\
\text { north of disposal cell } \\
\text { east of disposal cell (Area C) } \\
\text { north of disposal cell }\end{array}$ & $\begin{array}{l}1993 \\
1993 \\
1993 \\
1993 \\
1986-1993 \\
1986-1993 \\
1986-1993 \\
1993 \\
1993 \\
1993 \\
1993\end{array}$ & $\begin{array}{r}1 \\
1 \\
1 \\
1 \\
15 \\
15 \\
16 \\
\text { dry } \\
1 \\
1 \\
1\end{array}$ & $\begin{array}{l}14.0(\mathrm{a}) \\
14.0(\mathrm{a}) \\
20.0(\mathrm{a}) \\
21.0(\mathrm{a}) \\
14.3-19.3 \\
7.0-12.0 \\
4.5-14.5 \\
18.0-23.0 \\
8.0-13.0 \\
12.0-17.0 \\
12.5-17.5\end{array}$ \\
\hline \multicolumn{5}{|l|}{ Bedrock } \\
\hline \multicolumn{5}{|c|}{ Background wells } \\
\hline $\begin{array}{l}503 \\
504\end{array}$ & $\begin{array}{l}\text { southwest of disposal cell } \\
\text { southwest of disposal cell }\end{array}$ & $\begin{array}{l}1993 \\
1986-1993\end{array}$ & $\begin{array}{r}1 \\
16\end{array}$ & $\begin{array}{l}112.9(a) \\
19.9-24.9\end{array}$ \\
\hline \multicolumn{5}{|c|}{ Downgradient wells } \\
\hline $\begin{array}{l}502 \\
505 \\
506 \\
507 \\
508 \\
509 \\
510\end{array}$ & $\begin{array}{l}\text { across river from disposal cell } \\
\text { across river from disposal cell } \\
\text { northwest of disposal cell } \\
\text { west of disposal cell } \\
\text { north of disposal cell } \\
\text { east of disposal cell (Area C) } \\
\text { north of disposal cell }\end{array}$ & $\begin{array}{l}1993 \\
1986-1993 \\
1986-1993 \\
1993 \\
1993 \\
1993 \\
1993\end{array}$ & $\begin{array}{r}1 \\
16 \\
16 \\
1 \\
1 \\
1 \\
1\end{array}$ & $\begin{array}{l}82.3(a) \\
25.4-35.4 \\
21.0-26.0 \\
38.0-43.0 \\
27.0-32.0 \\
29.5-34.5 \\
32.0-37.0\end{array}$ \\
\hline
\end{tabular}


Table 3.1 Monitor well and surface water sampling information, Canonsburg, Pennsylvania, site (Concluded)

\begin{tabular}{llc}
\hline Well Number $\quad$ Location & \multicolumn{1}{c}{$\begin{array}{c}\text { Years } \\
\text { sampled }\end{array}$} & $\begin{array}{c}\text { Number } \\
\text { of rounds }\end{array}$ \\
\hline $\begin{array}{l}\text { Surface water - Chartiers Creek } \\
\text { Downstream locations }\end{array}$ & & \\
\hline & & \\
602 east of site, north of railroad & $1989-1993$ & 9 \\
604 east of site, north of Strabane Ave & 1993 & 1 \\
605 north of site & 1993 & 1 \\
606 north of site & 1993 & 1 \\
Upstream location & 1993 & \\
601 southwest of site, north of railroad & & 9 \\
\hline
\end{tabular}

(a) Total depth (ft) 
site. Therefore, monitor well 504 was selected to represent background bedrock ground water conditions at Canonsburg.

A trilinear plot of the background water from the two units (Figure 3.1) shows that ground water in monitor well 504 has a mixed anionic composition with roughly equal amounts of chloride, sulfate, and bicarbonate. Calcium is the dominant cation. Conversely, the ground water from background well 410 has a mixed cationic composition with roughly equal amounts of sodium and calcium and slightly less magnesium. Sulfate, with slightly less bicarbonate and chloride, dominates the anionic composition of this ground water. As can be seen from the concentration versus time plots (Figure 3.2, plots $a, b, c$, and d), the $\mathrm{pH}$, alkalinity, sulfate concentrations, and total dissolved solids (TDS) are much higher in the bedrock background ground water. Conversely, iron and manganese concentrations are higher in ground water from the shallow unconsolidated unit than from the deeper bedrock unit (Figure 3.2, plots e and f).

\subsection{MAGNITUDE AND EXTENT OF SITE-RELATED GROUND WATER} CONTAMINATION

Geochemical and statistical analyses of the available chemical data from the unconsolidated and bedrock units suggest that major, minor, and trace constituents are elevated in some downgradient wells relative to background ground water (DOE, 1995).

\subsubsection{Unconsolidated materials}

Wells completed in the unconsolidated material with the highest levels of siterelated contamination are 412, 413, and 414 (Figures 3.3 and 3.4). Wells 412 and 413 are within the restricted zone and, as discussed in Section 2.4, contamination in these wells is not expected to migrate to potentially accessible ground water areas. Monitor wells 414 and 424 are in Area $C$, southeast of the disposal cell. One round of ground water samples was collected from monitor well 424, within 2 weeks after the well was completed. Comparison of water quality data from monitor wells 414 and 424 indicates that monitor well 414 shows higher levels of some constituents (boron, potassium, manganese, molybdenum, uranium, lead-210, radium-226, and thorium-230) than are seen in monitor well 424 . Conversely, monitor well 424 shows higher levels of other constituents (notably, ammonium, arsenic, calcium, chloride, iron, sodium, sulfate, and radium-228) (Table 3.2). Because only one round of data is available from monitor well 424, these results have not been confirmed. For this reason, and because Area $C$ is being considered for public use, discussion of contamination will focus on monitor well 414.

A trilinear diagram (Figure 3.1) can illustrate some of the major elemental differences between background ground water from upgradient monitor well 410 and contaminated ground water from downgradient well 414 in unconsolidated materials. The contaminated ground water of the shallow 


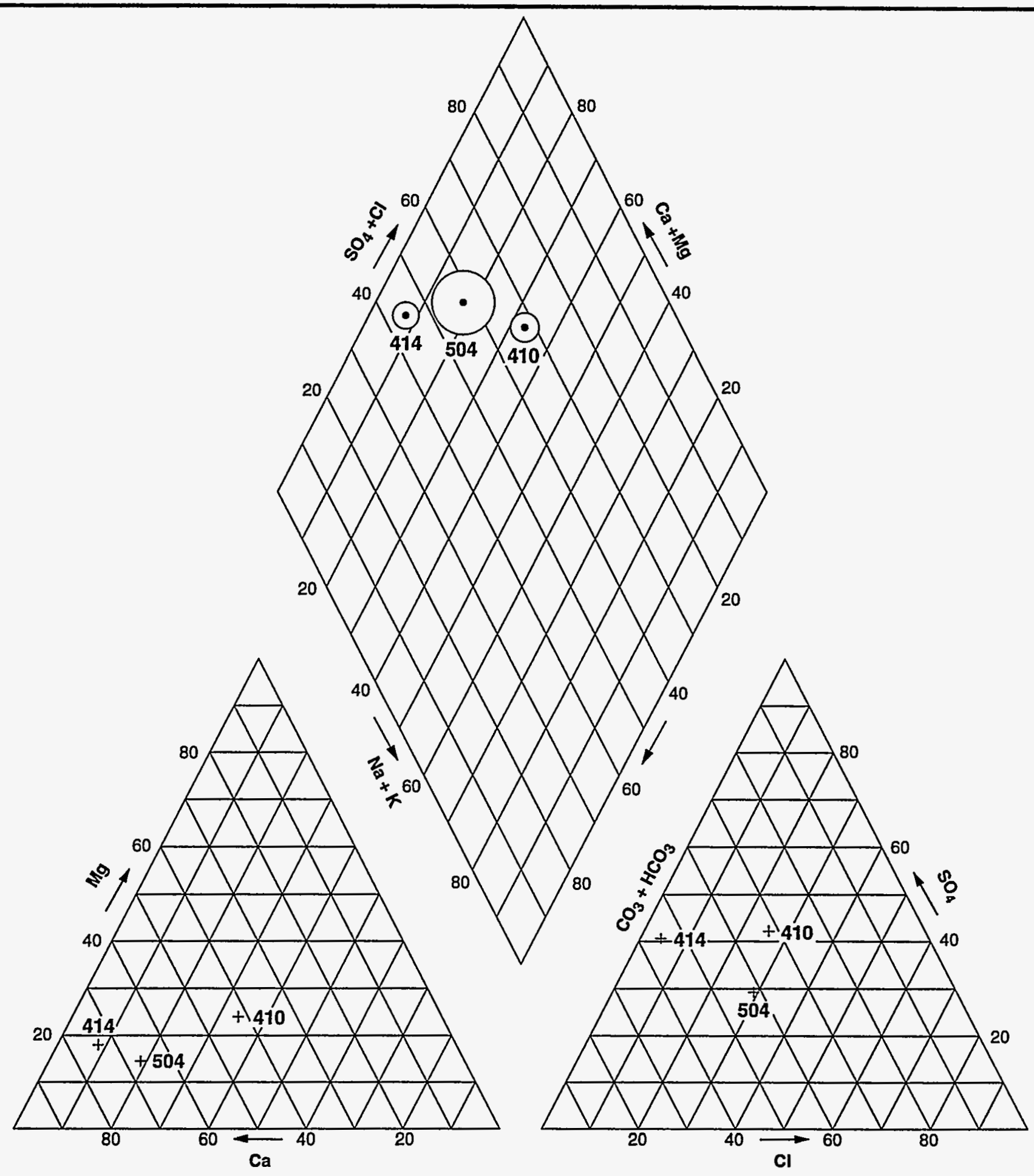

Cations

LEGEND

NUMBER TDS WELLNAME

$504 \quad 829$ BACKGROUND WELL

414380 DOWNGRADIENT WELL

$410 \quad 420$ BACKGROUND WELL

$2000 \mathrm{mg} / \mathrm{L}$ per INCH

FIGURE 3.1

TRILINEAR PLOT SHOWING ANION-CATION COMPOSITION OF BACKGROUND WELLS 410 AND 504 AND DOWNGRADIENT WELL 414 CANONSBURG, PENNSYLVANIA, SITE 

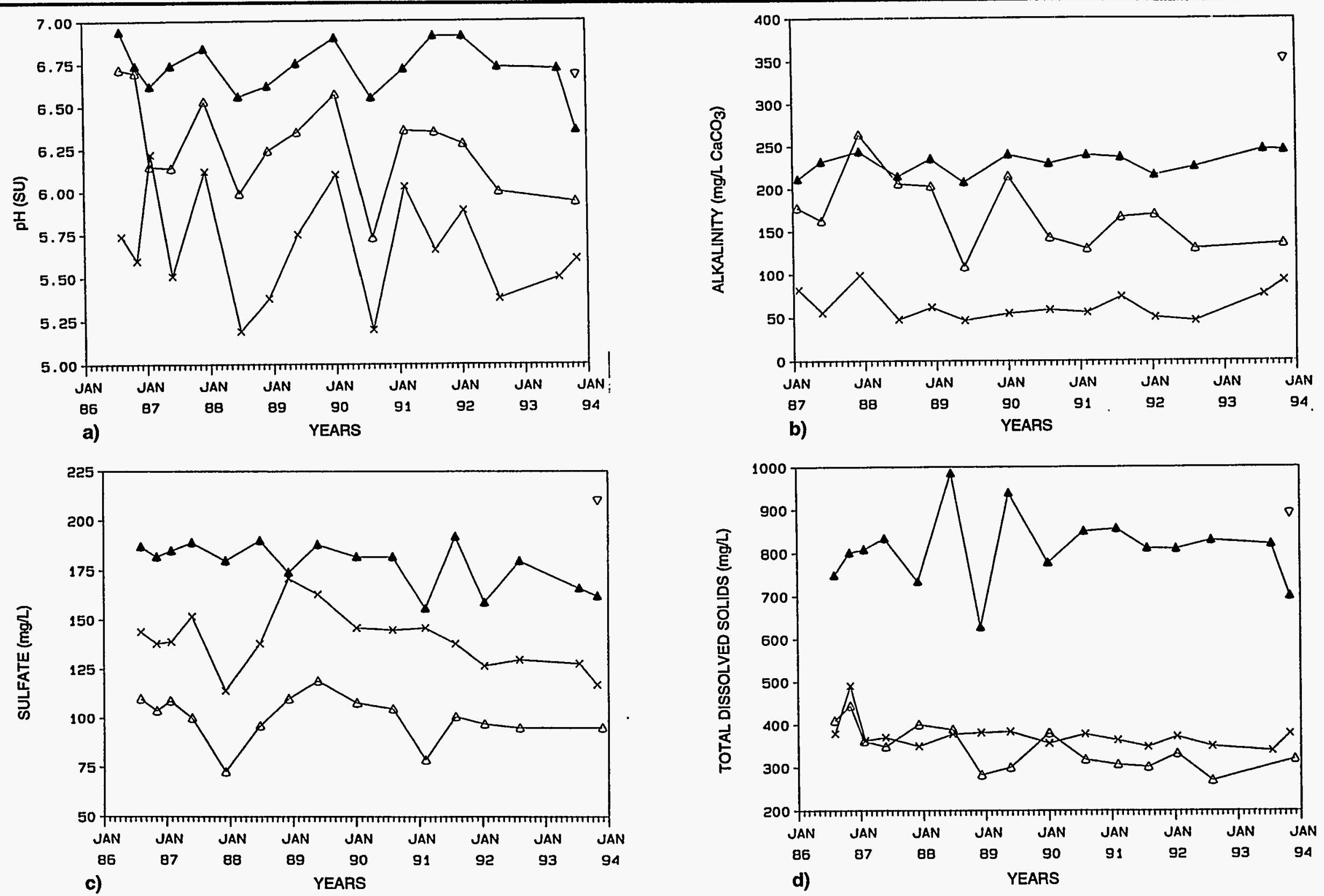

FIGURE 3.2

GROUND WATER PARAMETER VERSUS TIME PLOTS OF WELLS

410, 414, 504, AND 509

CANONSBURG, PENNSYLVANIA, SITE

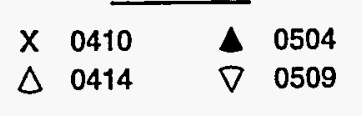




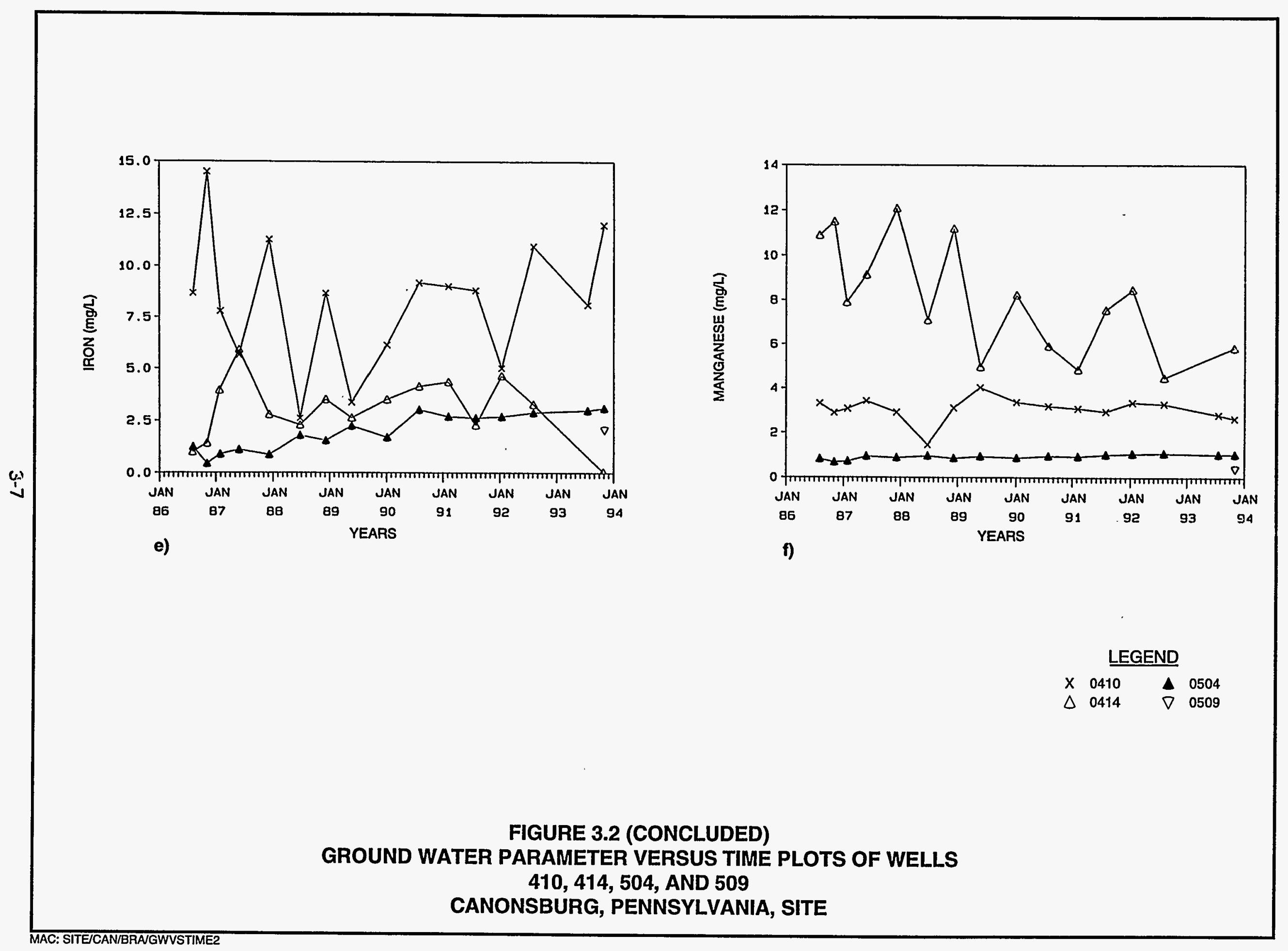




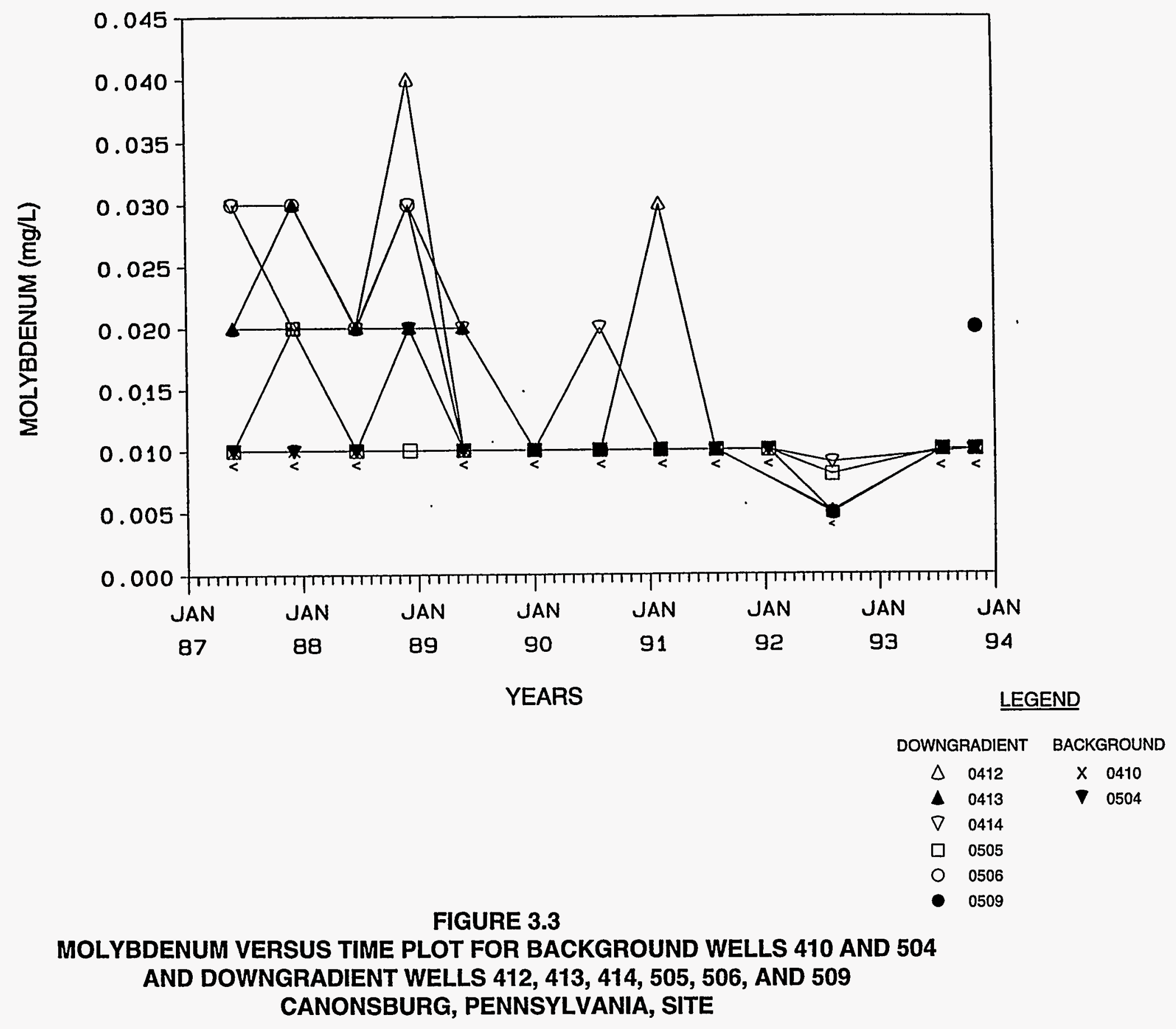




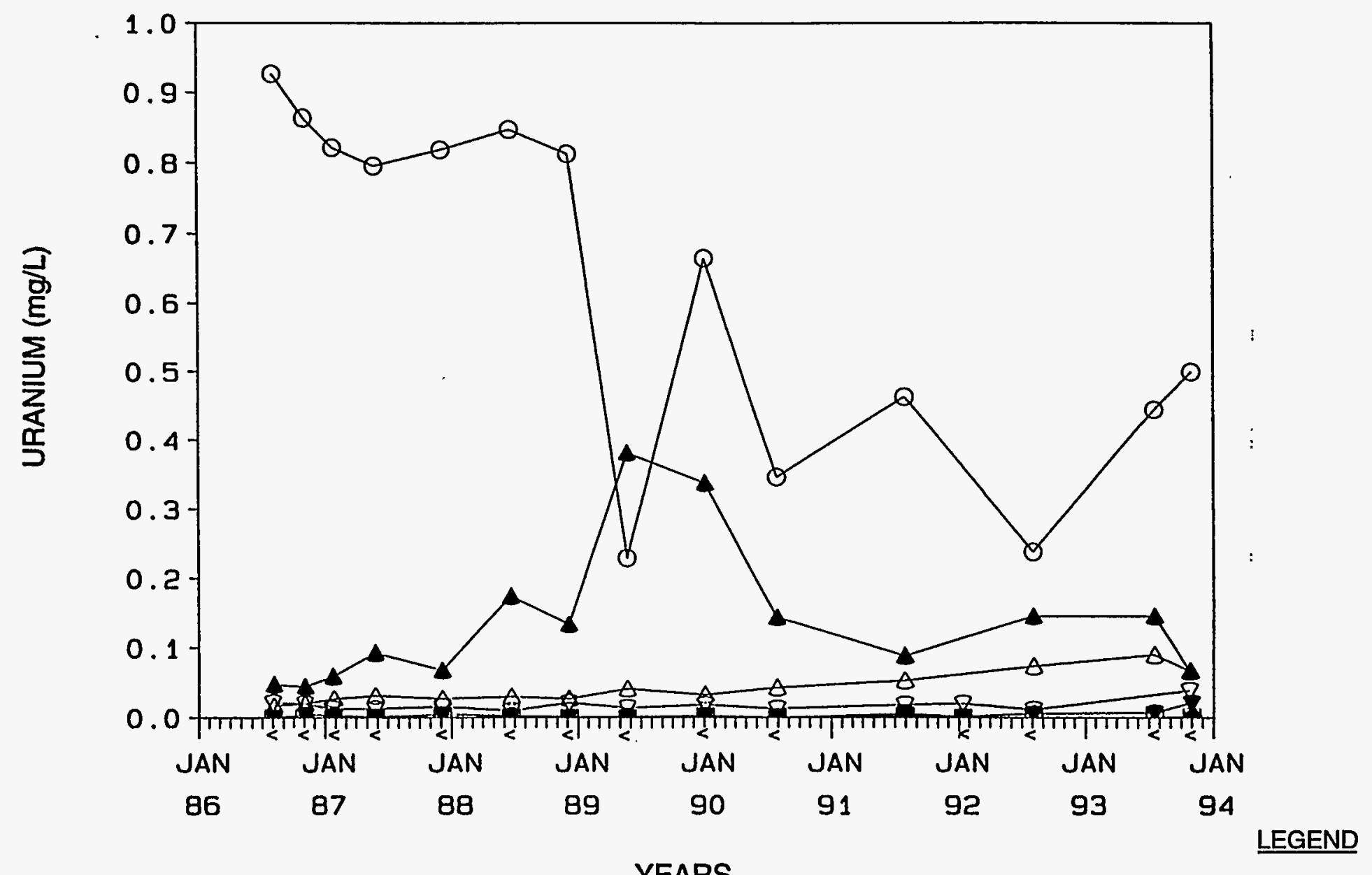

YEARS

FIGURE 3.4

URANIUM VERSUS TIME PLOT FOR BACKGROUND WELLS 410 AND 504

AND DOWNGRADIENT WELLS 412, 413, 414, 505, 506, AND 509

CANONSBURG, PENNSYLVANIA, SITE 
Table 3.2 Comparison of background and on-site ground water quality data from the unconsolidated materials, Canonsburg, Pennsylvania, site (1986-1993, filtered water samples)

\begin{tabular}{|c|c|c|c|c|}
\hline Parameter & $\begin{array}{c}\text { Above } \\
\text { detection }\end{array}$ & Minimum & Median $^{b}$ & Maximum \\
\hline Inorganics & & & $\mathrm{mg} / \mathrm{L}$ & \\
\hline 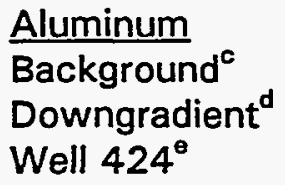 & $\begin{array}{l}4 / 7 \\
5 / 7 \\
0 / 1\end{array}$ & $\begin{array}{r}0.03 \\
<0.05 \\
-\end{array}$ & $\begin{array}{r}0.03 \\
0.08 \\
<0.05\end{array}$ & $\begin{array}{l}0.30 \\
0.30 \\
-\end{array}$ \\
\hline $\begin{array}{l}\text { Ammonium }{ }^{f, g} \\
\text { Background } \\
\text { Downgradient } \\
\text { Well } 4240\end{array}$ & $\begin{array}{l}1 / 6 \\
5 / 5 \\
1 / 1\end{array}$ & $\begin{array}{c}<0.06 \\
0.35 \\
-\end{array}$ & $\begin{array}{l}0.80 \\
12.0\end{array}$ & $\begin{array}{l}0.10 \\
1.30 \\
-\end{array}$ \\
\hline $\begin{array}{l}\text { Antimony } \\
\text { Background } \\
\text { Downgradient } \\
\text { Well } 424\end{array}$ & $\begin{array}{l}0 / 2 \\
0 / 2 \\
\text { NA }\end{array}$ & $\begin{array}{l}<0.003 \\
<0.003\end{array}$ & $\begin{array}{l}- \\
-\end{array}$ & $\begin{array}{l}<0.003 \\
<0.003 \\
-\end{array}$ \\
\hline $\begin{array}{l}\text { Arsenic } \\
\text { Background } \\
\text { Downgradient } \\
\text { Well } 424\end{array}$ & $\begin{array}{l}1 / 7 \\
3 / 7 \\
1 / 1\end{array}$ & $\begin{array}{c}<0.001 \\
<0.001 \\
\end{array}$ & $\begin{array}{l}- \\
- \\
0.008\end{array}$ & $\begin{array}{c}<0.01 \\
<0.01 \\
-\end{array}$ \\
\hline $\begin{array}{l}\text { Barium } \\
\text { Background } \\
\text { Downgradient } \\
\text { Well } 424\end{array}$ & $\begin{array}{l}2 / 2 \\
2 / 2 \\
\text { NA }\end{array}$ & $\begin{array}{l}0.10 \\
0.10 \\
-\end{array}$ & $\begin{array}{l}0.20 \\
0.15 \\
-\end{array}$ & $\begin{array}{l}0.30 \\
0.20 \\
-\end{array}$ \\
\hline $\begin{array}{l}\text { Bervllium } \\
\text { Background } \\
\text { Downgradient } \\
\text { Well } 424\end{array}$ & $\begin{array}{l}0 / 2 \\
0 / 2 \\
N A\end{array}$ & $\begin{array}{l}<0.005 \\
<0.005 \\
-\end{array}$ & $\begin{array}{l}- \\
-\end{array}$ & $\begin{array}{c}<0.01 \\
<0.01 \\
-\end{array}$ \\
\hline $\begin{array}{l}\text { Boron }^{g} \\
\text { Background } \\
\text { Downgradient } \\
\text { Well } 424\end{array}$ & $\begin{array}{l}4 / 7 \\
6 / 7 \\
0 / 1\end{array}$ & $\begin{array}{r}0.03 \\
<0.10 \\
-\end{array}$ & $\begin{array}{l}0.03 \\
0.23 \\
<0.2\end{array}$ & $\begin{array}{l}0.20 \\
0.39 \\
-\end{array}$ \\
\hline $\begin{array}{l}\text { Bromide } \\
\text { Background } \\
\text { Downgradient } \\
\text { Well } 424\end{array}$ & $\begin{array}{l}1 / 1 \\
0 / 1 \\
1 / 1\end{array}$ & $\begin{array}{l}0.10 \\
<0.10 \\
-\end{array}$ & $\begin{array}{l}- \\
- \\
0.2\end{array}$ & $\begin{array}{r}0.10 \\
<0.10 \\
-\end{array}$ \\
\hline $\begin{array}{l}\text { Cadmium } \\
\text { Background } \\
\text { Downgradient } \\
\text { Well } 424\end{array}$ & $\begin{array}{l}0 / 5 \\
0 / 5 \\
0 / 1\end{array}$ & $\begin{array}{c}<0.001 \\
<0.001 \\
-\end{array}$ & $\begin{array}{c}- \\
- \\
<0.001 \\
\end{array}$ & $\begin{array}{l}<0.001 \\
<0.001 \\
\quad-\end{array}$ \\
\hline
\end{tabular}


Table 3.2 Comparison of background and on-site ground water quality data from the unconsolidated materials, Canonsburg, Pennsylvania, site (1986-1993, filtered water samples) (Continued)

\begin{tabular}{|c|c|c|c|c|}
\hline Parameter & $\begin{array}{c}\text { Above } \\
\text { detection }\end{array}$ & Minimum & Median $^{b}$ & Maximum \\
\hline & & & $\mathrm{mg} / \mathrm{L}$ & \\
\hline 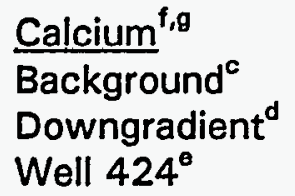 & $\begin{array}{c}11 / 11 \\
10 / 10 \\
1 / 1\end{array}$ & $\begin{array}{l}39.4 \\
70.0 \\
-\end{array}$ & $\begin{array}{l}43.6 \\
74.0 \\
96.7\end{array}$ & $\begin{array}{c}50.1 \\
92.8 \\
-\end{array}$ \\
\hline $\begin{array}{l}\text { Chloride } \\
\text { Background }^{f} \\
\text { Downgradient } \\
\text { Well } 424\end{array}$ & $\begin{array}{c}11 / 11 \\
10 / 10 \\
1 / 1\end{array}$ & $\begin{array}{c}36.0 \\
2.10 \\
-\end{array}$ & $\begin{array}{c}43.0 \\
3.85 \\
136\end{array}$ & $\begin{array}{c}51.9 \\
7.20 \\
-\end{array}$ \\
\hline $\begin{array}{l}\text { Chromium }^{f} \\
\text { Background }^{\prime} \\
\text { Downgradient } \\
\text { Well } 424\end{array}$ & $\begin{array}{l}1 / 6 \\
1 / 6 \\
0 / 1\end{array}$ & $\begin{array}{c}<0.01 \\
<0.01 \\
\end{array}$ & $\begin{array}{c}- \\
- \\
<0.01\end{array}$ & $\begin{array}{l}0.04 \\
0.05 \\
-\end{array}$ \\
\hline $\begin{array}{l}\text { Cobalt } \\
\text { Background } \\
\text { Downgradient } \\
\text { Well } 424\end{array}$ & $\begin{array}{l}1 / 5 \\
0 / 5 \\
0 / 1\end{array}$ & $\begin{array}{r}0.03 \\
<0.03 \\
-\end{array}$ & $\begin{array}{c}- \\
- \\
<0.05\end{array}$ & $\begin{array}{c}<0.05 \\
<0.05 \\
-\end{array}$ \\
\hline $\begin{array}{l}\text { Copper } \\
\text { Background } \\
\text { Downgradient } \\
\text { Well } 424\end{array}$ & $\begin{array}{l}1 / 2 \\
1 / 2 \\
\text { NA }\end{array}$ & $\begin{array}{c}<0.02 \\
<0.02 \\
-\end{array}$ & - & $\begin{array}{l}0.02 \\
0.02 \\
-\end{array}$ \\
\hline $\begin{array}{l}\text { Cyanide }^{f} \\
\text { Background } \\
\text { Downgradient } \\
\text { Well } 424\end{array}$ & $\begin{array}{l}0 / 5 \\
2 / 5 \\
\text { NA }\end{array}$ & $\begin{array}{c}<0.01 \\
<0.01 \\
-\end{array}$ & - & $\begin{array}{c}<0.01 \\
0.01 \\
-\end{array}$ \\
\hline $\begin{array}{l}\text { Fluoride } \\
\text { Background } \\
\text { Downgradient } \\
\text { Well } 424\end{array}$ & $\begin{array}{l}7 / 7 \\
7 / 7 \\
1 / 1\end{array}$ & $\begin{array}{l}0.10 \\
0.14 \\
-\end{array}$ & $\begin{array}{l}0.14 \\
0.19 \\
0.1\end{array}$ & $\begin{array}{l}0.20 \\
0.30 \\
-\end{array}$ \\
\hline $\begin{array}{l}\text { Iron }^{f} \\
\text { Background } \\
\text { Downgradient } \\
\text { Well } 424\end{array}$ & $\begin{array}{c}11 / 11 \\
10 / 10 \\
1 / 1\end{array}$ & $\begin{array}{l}2.63 \\
0.07 \\
-\end{array}$ & $\begin{array}{r}8.68 \\
3.40 \\
22.3\end{array}$ & $\begin{array}{c}12.0 \\
4.66 \\
-\end{array}$ \\
\hline $\begin{array}{l}\text { Lead }^{f} \\
\text { Background } \\
\text { Downgradient } \\
\text { Well } 424\end{array}$ & $\begin{array}{l}0 / 11 \\
0 / 10 \\
0 / 1\end{array}$ & $\begin{array}{c}<0.001 \\
<0.001 \\
-\end{array}$ & $\begin{array}{c}- \\
- \\
<0.003\end{array}$ & $\begin{array}{c}<0.01 \\
<0.01 \\
-\end{array}$ \\
\hline
\end{tabular}


Table 3.2 Comparison of background and on-site ground water quality data from the unconsolidated materials, Canonsburg, Pennsyivania, site (1986-1993, filtered water samples) (Continued)

\begin{tabular}{|c|c|c|c|c|}
\hline Parameter & $\begin{array}{c}\text { Above } \\
\text { detection }\end{array}$ & Minimum & Median $^{b}$ & Maximum \\
\hline & & & $\mathrm{mg} / \mathrm{L}$ & \\
\hline $\begin{array}{l}\text { Magnesium }^{f} \\
\text { Background } \\
\text { Downgradient } \\
\text { Well } 424\end{array}$ & $\begin{array}{c}11 / 11 \\
10 / 10 \\
1 / 1\end{array}$ & $\begin{array}{c}14.1 \\
9.45 \\
-\end{array}$ & $\begin{array}{l}15.3 \\
10.3 \\
26.3\end{array}$ & $\begin{array}{l}17.0 \\
12.6 \\
-\end{array}$ \\
\hline 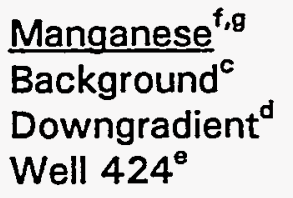 & $\begin{array}{c}11 / 11 \\
10 / 10 \\
1 / 1\end{array}$ & $\begin{array}{l}1.47 \\
4.50 \\
-\end{array}$ & $\begin{array}{l}3.12 \\
6.52 \\
6.43\end{array}$ & $\begin{array}{l}4.05 \\
11.2 \\
-\end{array}$ \\
\hline $\begin{array}{l}\text { Mercury } \\
\text { Background } \\
\text { Downgradient } \\
\text { Well } 424\end{array}$ & $\begin{array}{l}0 / 2 \\
0 / 2 \\
\text { NA }\end{array}$ & $\begin{array}{l}<0.0002 \\
<0.0002 \\
-\end{array}$ & $\begin{array}{l}- \\
-\end{array}$ & $\begin{array}{l}<0.0002 \\
<0.0002 \\
-\end{array}$ \\
\hline $\begin{array}{l}\text { Molybdenum }^{f, g} \\
\text { Background } \\
\text { Downgradient } \\
\text { Well } 424\end{array}$ & $\begin{array}{l}1 / 11 \\
5 / 10 \\
0 / 1\end{array}$ & $\begin{array}{c}<0.004 \\
0.009 \\
-\end{array}$ & $\begin{array}{c}- \\
- \\
<0.01\end{array}$ & $\begin{array}{l}0.02 \\
0.03 \\
-\end{array}$ \\
\hline $\begin{array}{l}\text { Nickel } \\
\text { Background } \\
\text { Downgradient } \\
\text { Well } 424\end{array}$ & $\begin{array}{l}2 / 5 \\
1 / 5 \\
0 / 1\end{array}$ & $\begin{array}{c}<0.01 \\
<0.01 \\
-\end{array}$ & $\begin{array}{c}- \\
- \\
<0.04\end{array}$ & $\begin{array}{r}0.05 \\
<0.04 \\
-\end{array}$ \\
\hline $\begin{array}{l}\text { Nitrate }^{f} \\
\text { Background } \\
\text { Downgradient } \\
\text { Well } 424\end{array}$ & $\begin{array}{l}1 / 10 \\
2 / 10 \\
0 / 1\end{array}$ & $\begin{array}{c}<0.10 \\
<0.10 \\
-\end{array}$ & $\begin{array}{c}- \\
<1.0\end{array}$ & $\begin{array}{c}<1.0 \\
1.2 \\
-\end{array}$ \\
\hline $\begin{array}{l}\text { Phosphate } \\
\text { Background } \\
\text { Downgradient } \\
\text { Well } 424\end{array}$ & $\begin{array}{l}1 / 3 \\
1 / 3 \\
1 / 1\end{array}$ & $\begin{array}{c}<0.01 \\
<0.01 \\
-\end{array}$ & $\begin{array}{l}- \\
- \\
0.40\end{array}$ & $\begin{array}{l}0.20 \\
0.20 \\
-\end{array}$ \\
\hline $\begin{array}{l}\text { Potassium }^{f, g} \\
\text { Background } \\
\text { Downgradient } \\
\text { Well } 424\end{array}$ & $\begin{array}{c}11 / 11 \\
10 / 10 \\
1 / 1\end{array}$ & $\begin{array}{l}0.97 \\
1.82 \\
-\end{array}$ & $\begin{array}{l}1.2 \\
2.1 \\
3.2\end{array}$ & $\begin{array}{l}2.5 \\
4.6 \\
-\end{array}$ \\
\hline $\begin{array}{l}\text { Selenium }^{f} \\
\text { Background } \\
\text { Downgradient } \\
\text { Well } 424\end{array}$ & $\begin{array}{c}0 / 11 \\
0 / 10 \\
0 / 1\end{array}$ & $\begin{array}{c}<0.001 \\
<0.001 \\
-\end{array}$ & $<0.005$ & $\begin{array}{l}<0.005 \\
<0.005 \\
-\end{array}$ \\
\hline
\end{tabular}


Table 3.2 Comparison of background and on-site ground water quality data from the unconsolidated materials, Canonsburg, Pennsylvania, site (1986-1993, filtered water samples) (Continued)

\begin{tabular}{|c|c|c|c|c|}
\hline Parameter & $\begin{array}{c}\text { Above } \\
\text { detection }\end{array}$ & Minimum & Median $^{\mathbf{b}}$ & Maximum \\
\hline & & & $\mathrm{mg} / \mathrm{L}$ & \\
\hline \multicolumn{5}{|l|}{ Silica } \\
\hline$\overline{\text { Background }}$ & $3 / 3$ & 6.0 & 6.8 & 13.8 \\
\hline Downgradient & $3 / 3$ & 3.9 & 5.0 & 11.6 \\
\hline Well 424 & $1 / 1$ & - & 12.1 & - \\
\hline \multicolumn{5}{|l|}{ Silver } \\
\hline$\overline{\text { Background }}$ & $0 / 2$ & $<0.01$ & - & $<0.01$ \\
\hline Downgradient & $0 / 2$ & $<0.01$ & - & $<0.01$ \\
\hline Well 424 & NA & - & - & - \\
\hline \multicolumn{5}{|l|}{ Sodium $^{\dagger}$} \\
\hline$\overline{\text { Background }}^{c}$ & $11 / 11$ & 38 & 43.9 & 50.1 \\
\hline Downgradient ${ }^{d}$ & $10 / 10$ & 5.9 & 7.83 & 17.5 \\
\hline Well $424^{\circ}$ & $1 / 1$ & - & 157 & - \\
\hline \multicolumn{5}{|l|}{ Strontium $^{f, g}$} \\
\hline Background & $2 / 3$ & $<0.10$ & 0.10 & 0.13 \\
\hline Downgradient & $3 / 3$ & 0.20 & 0.29 & 0.30 \\
\hline Well 424 & $1 / 1$ & - & 0.32 & - \\
\hline \multicolumn{5}{|l|}{ Sulfate $^{f}$} \\
\hline$\overline{\text { Background }}$ & $11 / 11$ & 117 & 138 & 171 \\
\hline Downgradient & $10 / 10$ & 79 & 99 & 119 \\
\hline Well 424 & $1 / 1$ & - & 210 & - \\
\hline \multicolumn{5}{|l|}{ Sulfur } \\
\hline$\overline{\text { Background }}$ & $0 / 1$ & $<0.10$ & - & $<0.10$ \\
\hline Downgradient & $0 / 2$ & $<0.10$ & - & $<0.10$ \\
\hline Well 424 & NA & - & - & - \\
\hline \multicolumn{5}{|l|}{ Thallium $^{f}$} \\
\hline$\overline{\text { Background }}$ & $0 / 6$ & $<0.01$ & - & $<0.10$ \\
\hline Downgradient & $0 / 6$ & $<0.01$ & - & $<0.10$ \\
\hline Well 424 & NA & - & - & - \\
\hline \multicolumn{5}{|l|}{ Tin } \\
\hline Background & $1 / 2$ & $<0.005$ & - & 0.011 \\
\hline Downgradient & $0 / 2$ & $<0.005$ & - & $<0.005$ \\
\hline Well 424 & NA & - & - & - \\
\hline \multicolumn{5}{|l|}{ Uranium $^{f, g}$} \\
\hline Background & $2 / 10$ & $<0.001$ & - & 0.003 \\
\hline Downgradient & $9 / 9$ & 0.01 & 0.02 & 0.04 \\
\hline Well 424 & $0 / 1$ & - & $<0.001$ & - \\
\hline
\end{tabular}


Table 3.2 Comparison of background and on-site ground water quality data from the unconsolidated materials, Canonsburg, Pennsylvania, site (1986-1993, filtered water samples) (Concluded)

\begin{tabular}{|c|c|c|c|c|}
\hline Parameter & $\begin{array}{c}\text { Above } \\
\text { detection }\end{array}$ & Minimum & Median' & Maximum \\
\hline & & & $\mathrm{mg} / \mathrm{L}$ & \\
\hline \multicolumn{5}{|l|}{ Vanadium $^{f}$} \\
\hline$\overline{\text { Background }}$ & $2 / 10$ & $<0.001$ & - & 0.02 \\
\hline Downgradient & $2 / 9$ & $<0.001$ & - & 0.02 \\
\hline Well 424 & $0 / 1$ & - & $<0.01$ & - \\
\hline \multicolumn{5}{|l|}{ Zinc } \\
\hline$\overline{\text { Background }}$ & $7 / 7$ & 0.033 & 0.079 & 0.157 \\
\hline Downgradient & $6 / 7$ & $<0.005$ & 0.014 & 0.051 \\
\hline Well 424 & $0 / 1$ & - & $<0.005$ & - \\
\hline Radionuclides & & \multicolumn{3}{|c|}{$\overline{p C i / L}$} \\
\hline \multicolumn{5}{|l|}{ Lead-210 } \\
\hline$\overline{\text { Background }}^{c}$ & $1 / 1$ & 1.1 & - & 1.1 \\
\hline Downgradient ${ }^{d}$ & $2 / 2$ & ND & 2.5 & 5.0 \\
\hline Well $424^{\circ}$ & NA & - & - & - \\
\hline \multicolumn{5}{|l|}{ Polonium } \\
\hline Background & $1 / 1$ & 0.10 & - & 0.10 \\
\hline Downgradient & $2 / 2$ & ND & 0.05 & 0.10 \\
\hline Well 424 & NA & - & - & - \\
\hline \multicolumn{5}{|l|}{ Radium-226 } \\
\hline$\overline{\text { Background }}$ & $6 / 6$ & ND & 0.105 & 0.30 \\
\hline Downgradient & $5 / 5$ & ND & 0.10 & 0.70 \\
\hline Well 424 & $1 / 1$ & - & 0.2 & - \\
\hline \multicolumn{5}{|l|}{ Radium-228 } \\
\hline$\overline{\text { Background }}$ & $6 / 6$ & ND & 0.60 & 1.2 \\
\hline Downgradient & $5 / 5$ & ND & 1.15 & 2.3 \\
\hline Well 424 & $1 / 1$ & - & 3.8 & - \\
\hline \multicolumn{5}{|l|}{ Thorium-230 } \\
\hline Background & $2 / 2$ & ND & 0.70 & 1.4 \\
\hline Downgradient & $3 / 3$ & ND & 0.10 & 0.7 \\
\hline Well 424 & $1 / 1$ & - & 0.2 & - \\
\hline
\end{tabular}

Above detection $=$ Number of samples with detectable concentration/total number of samples.

'Median is the 50th percentile of the data. The median cannot be calculated if 50 percent or more of the data are below detection. For parameters having only one round of sampling data, the single reported value is listed in the median column.

${ }^{\circ} D O E$ well 410 was used as background.

${ }^{d} D O E$ well 414 is located in Area $C$ and was used as the downgradient well.

"Monitor well 424 is in Area $C$ and is indicative of downgradient conditions but, with only one sampling round, could not be included in the statistical analysis. The single value for each parameter from this well is reported in the median column.

'Data collected from 1988 through 1993 are summarized for these parameters. For all other parameters, all available data from 1986 through 1993 are summarized.

'Concentrations are statistically elevated in well 414 above background.

NA - not applicable.

ND - not detected. 
unconsolidated unit is relatively more calcium-rich and bicarbonate-rich and chloride-poor than the background ground water from monitor well 410 . Concentration versus time plots (Figures 3.2, 3.3, and 3.4) show elevated levels of manganese, molybdenum, and uranium in monitor well 414 relative to background. Note that one component of the unconsolidated unit is iron slag, which was used as fill material (DOE, 1983b). This material contains high concentrations of iron and manganese and its presence in the subsurface could affect iron and manganese concentrations in the ground water. Table 3.2 is a statistical summary of ground water data from background and select downgradient wells in the unconsolidated materials for filtered water samples.

\subsubsection{Bedrock unit}

Well 505 shows no sign of contamination. Uranium concentrations in well 506 have been above 0.2 milligrams per liter (mg/L) since sampling began in 1986 (Figure 3.4). This monitor well was installed in an area containing contaminated material that was not put into the disposal cell (this contaminated material is in a separate covered area), apparently resulting in the locally elevated concentrations of uranium. Monitor well 510 was installed just downgradient from well 506 to verify a possible local source of contamination. The concentration of uranium in ground water from well 510 is low lless than the detection limit), indicating that the above assumption is probably correct. Also, the apparent lack of lateral hydraulic continuity of ground water in the shallow bedrock may be substantiated by these results, indicating that even with an elevated source area, migration of contaminants in this area is minimal. Contamination appears to have affected no other well in the bedrock unit. Comparing data from well 509 (located in Area C) with data from background well 504, it appears that over time, concentrations in well 509 generally fall within the range of levels found in well 504 (DOE, 1995). Thus, current data indicate that the bedrock unit is not contaminated except in the immediate area of monitor well 506. The site will continue to be monitored, and risks can be reevaluated if there is any indication that contamination has migrated into the bedrock unit.

\subsubsection{Summary of site-related contamination}

It is not possible to delineate a plume due to the variable nature of contamination across the site. However, contamination is limited to shallow ground water in the areas immediately adjacent to and beneath the disposal cell and ground water in Area C. After the extent of contamination was determined, 1986 through 1993 water quality data were evaluated to identify statistically those constituents elevated above background concentrations in the alluvial aquifer (DOE, 1995). Qualitative trend analysis of concentration versus time for all parameters indicated that pre-1988 data generally have greater variability and greater number of anomalies than data collected from 1988 through 1993 (TAC, 1994a). Moreover, the recent data are considered to be more representative of current ground water conditions. For these reasons, when possible, data from sampling events in 1988 through 1993 were preferentially 
used in the statistical evaluation over pre-1988 data. For many parameters, however, a limited number of sampling rounds from 1988 through 1993 did not allow for rigorous statistical comparisons. In these cases, data from all sampling events from 1986 through 1993 were used.

For each constituent, a Mann-Whitney test was conducted at the 0.05 level of significance (DOE, 1995). A significant Mann-Whitney test result indicates higher concentrations, on average, in the samples from well 414 than those in background well 410 . Table 3.2 is a summary of 1986 through 1993 water quality data in the alluvial aquifer. Those constituents for which only data from 1988 through 1993 were used are identified. Table 3.2 also identifies constituents statistically elevated above background levels.

\subsection{CONTAMINANTS OF POTENTIAL CONCERN}

The data presented in Sections 3.1 and 3.2 were used to compile a list of contaminants of potential concern for the assessment of human health or environmental risks at the Canonsburg site. In general, a contaminant was placed on the list of contaminants of potential concern if it was detected in excess of background levels as measured in upgradient monitor wells and if the site is a possible source for the contaminant.

Table 3.3 presents the list of contaminants of potential concern for ground water. The contaminants listed in column 1 represent those contaminants detected in the unconsolidated material in Area C (represented by well 414) that were statistically elevated from upgradient levels in the unconsolidated material (represented by well 410). These contaminants were further screened for their potential to affect human health in order to develop a final list of contaminants of potential concern for human health. Several constituents were screened out because they are essential nutrients present at levels within the nutritional ranges even when added to expected dietary intake. These constituents include calcium and potassium (DOE, 1994; 1995). Ammonium, boron, and strontium were eliminated as contaminants of potential concern based on low toxicity and/or intakes present at normal dietary levels relative to observed levels (DOE, 1995). Although ammonium is not considered a dietary component, it is produced in the human body at levels exceeding 4000 milligrams $(\mathrm{mg}$ ) per day (Summerskill and Wolpert, 1970), over 3 orders of magnitude more than would be expected from the ingestion of the maximum ammonium levels in well 414 $(1.3 \mathrm{mg} / \mathrm{L})$. Thus, the levels of ammonium in well 414 are not likely to be associated with adverse health effects.

Based on the screening, manganese, molybdenum, and uranium were chosen as final contaminants of potential concern for the human health risk assessment. Also, because uranium decays to radioactive progeny, Section 6.0 will evaluate the longer-lived radioactive isotopes of the uranium decay series (represented by lead-210, polonium-210, radium-226, and thorium-230). These seven constituents form the basis of the human health risk assessment for the unconsolidated material at the Canonsburg site. 
Table 3.3 Contaminants of potential concern in ground water, Canonsburg, Pennsylvania, site

\begin{tabular}{|c|c|c|c|}
\hline $\begin{array}{l}\text { Contaminant levels } \\
\text { exceeding background }\end{array}$ & $\begin{array}{l}\text { Contaminant levels } \\
\text { in nutritional range }\end{array}$ & $\begin{array}{l}\text { Contaminants of } \\
\text { low toxicity and/or } \\
\text { high dietary range }\end{array}$ & $\begin{array}{l}\text { Contaminants of } \\
\text { potential concern } \\
\text { for human health }\end{array}$ \\
\hline $\begin{array}{l}\text { Ammonium } \\
\text { Boron } \\
\text { Calcium } \\
\text { Manganese } \\
\text { Molybdenum } \\
\text { Potassium } \\
\text { Strontium } \\
\text { Uranium }\end{array}$ & $\begin{array}{l}\text { Calcium } \\
\text { Potassium }\end{array}$ & $\begin{array}{l}\text { Ammonium } \\
\text { Boron } \\
\text { Strontium }\end{array}$ & $\begin{array}{l}\text { Manganese } \\
\text { Molybdenum } \\
\text { Uranium }^{b}\end{array}$ \\
\hline
\end{tabular}

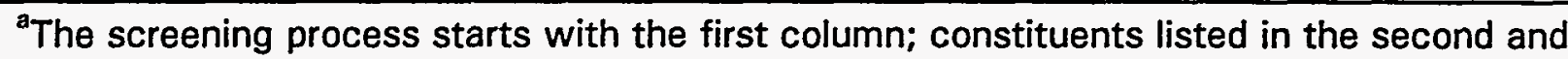
third columns were subtracted from the list of constituents in the first column; the remaining constituents form the list in the last column.

${ }^{b}$ The longer-lived radioactive isotopes of the uranium decay series are represented by radium-226, lead-210, polonium-210, and thorium-230. These radionuclides are evaluated as contaminants of potential concern.

Note: Based on data from well 414 located in the unconsolidated material in Area C.

Because ecological impacts differ from effects on human health, the complete list of contaminants was considered for the ecological evaluation in Section 7.0. In addition, due to other ecological-specific considerations, the ecological evaluation also assessed contaminants from wells 412 and 413 that exceeded background levels. Additional contaminants evaluated in the ecological assessment as a result of the inclusion of these two wells were arsenic, chloride, magnesium, sodium, and sulfate.

Some constituents (ammonium, arsenic, calcium, chloride, iron, sodium, sulfate, and radium-228) were observed at higher levels in ground water from monitor well 424 than 414 (Table 3.2). These constituents could potentially be included in the list presented in column 1 in Table 3.3 if analytical results of the only sampling round from monitor well 424 were used, without further sampling, to identify contaminants of potential concern. Ammonium, calcium, chloride, and sodium would be screened out from this list, because the levels at which they were detected are within either nutritional or normal dietary ranges, even when added to expected dietary intake (Gilman et al., 1990). Sulfate would be screened out because it is not toxic at detected levels $(210 \mathrm{mg} / \mathrm{L})$ (EPA, 1992). Potential presence of arsenic, iron, and radium-228 at these levels in well 424 needs to be further evaluated. 
At the Canonsburg site, ground water occurs in near-surface unconsolidated materials (including fill material) and in the underlying bedrock. As contaminants migrate through the ground water system, they interact with natural chemical variations in ground water at the site and with the matrix materials. These interactions will produce variations in contaminant concentrations that are not simply the result of physical dispersion. Contaminant interactions are strongly dependent on Eh and $\mathrm{pH}$ conditions, ionic strength (generally indicated by TDS), and the speciation of the elements of concern in ground water.

Water from background well 410 completed in the unconsolidated material shows $\mathrm{pH}$ in the range of 5.4 to 6.2 and redox potentials near 300 millivolts (mV). Water from downgradient well 414, also completed in the unconsolidated material, shows $\mathrm{pH}$ in the range of 5.7 to 6.7 and redox potentials near $400 \mathrm{mV}$. The downgradient well has a slightly higher $\mathrm{pH}$ and a slightly higher redox potential, but both waters are mildly acidic and oxidizing.

In contrast, water in the bedrock system, in addition to having substantially higher TDS, is reducing (Eh near $0 \mathrm{mV}$ ) and has a more variable $\mathrm{pH}$. The $\mathrm{pH}$ ranges from near 8.5 in well 505, completed in the bedrock unit across Chartiers Creek east of the site, to between 6.5 and 7.0 in background well 504 , completed in the bedrock unit at the same location as background well 410. Ground water from downgradient well 414 (January 1991 sampling round), completed in the unconsolidated material, was modeled using the geochemical code PHREEOE (Parkhurst et al., 1980) to predict which species of the contaminants of concern might be present in ground water at the site (DOE, 1995). Information for some species was taken from Brookins (1988). Table 3.4 presents results of the model.

\section{Manganese}

Manganese concentrations ranging from 4.85 to $12.1 \mathrm{mg} / \mathrm{L}$ have been observed in downgradient monitor well 414 . These values are significantly higher than those observed in background well 410 , which range from 1.47 to $3.42 \mathrm{mg} / \mathrm{L}$ (Figure 3.3). Water from both wells is mildly oxidizing (redox potentials near $300 \mathrm{mV}$ ), with pH values between 5 and 6 . Under these conditions, modeling with the geochemical code PHREEOE (Parkhurst et al., 1980) suggests that $\mathrm{Mn}^{2+}$ accounts for more than 90 molar percent of manganese species present (Table 3.4). $\mathrm{Mn}^{2+}$ is relatively mobile and the major decrease in manganese concentration will likely be adsorption onto matrix materials and dilution as ground water discharges into the adjacent Chartiers Creek. This creek is the primary discharge point for the shallow ground water system on the site.

Water from monitor well 505, completed in the bedrock ground water system, is relatively more reducing (redox potentials near $0 \mathrm{mV}$ ) and has a higher $\mathrm{pH}$ (near 8.5) compared with well $414(\mathrm{pH}$ near 6$)$. If there is flow from the shallow ground water table to the bedrock ground water system, creation of solid 
Table 3.4 Stable species of constituents of concern in the ground water system at the Canonsburg, Pennsylvania, site

\begin{tabular}{|c|c|c|c|}
\hline $\begin{array}{l}\text { Contaminant of } \\
\text { potential concern }\end{array}$ & Common name & $\begin{array}{l}\text { Identity of species } \\
\text { in ground water }\end{array}$ & $\begin{array}{c}\text { Approximate } \\
\text { molar percentage }\end{array}$ \\
\hline \multirow[t]{3}{*}{ Manganese } & Manganese ion & $\mathrm{Mn}^{2+}$ & 92 \\
\hline & Manganese sulfate & $\mathrm{MnSO}_{4} \mathrm{AQ}$ & 5 \\
\hline & Manganese bicarbonate & $\mathrm{MnHCO}_{3}{ }^{+}$ & 3 \\
\hline Molybdenum & Molybdate & $\mathrm{MoO}_{4}{ }^{2-}$ & 100 \\
\hline \multirow[t]{3}{*}{ Uranium } & Uranyl dicarbonate & $\mathrm{UO}_{2}\left(\mathrm{CO}_{3}\right)_{2}{ }^{2-}$ & 63 \\
\hline & Uranyl carbonate & $\mathrm{UO}_{2} \mathrm{CO}_{3} \mathrm{AQ}$ & 34 \\
\hline & Uranyl tricarbonate & $\mathrm{UO}_{2}\left(\mathrm{CO}_{3}\right)_{3}^{4-}$ & 3 \\
\hline \multirow[t]{4}{*}{ Lead-210 } & Lead carbonate & $\mathrm{PbCO}_{3} \mathrm{AQ}$ & 49 \\
\hline & Lead bicarbonate & $\mathrm{PbHCO}_{3}{ }^{+}$ & 26 \\
\hline & Lead ion & $\mathrm{Pb}^{2+}$ & 21 \\
\hline & Lead sulfate & $\mathrm{PbSO}_{4} \mathrm{AQ}$ & 5 \\
\hline Thorium $-230^{a}$ & Thorium hydroxide & $\mathrm{Th}(\mathrm{OH})_{3}{ }^{+}$ & Dominant \\
\hline Polonium-210 & Polonium ion & $\mathrm{Po}^{2+}$ & 100 \\
\hline Radium $^{\mathbf{a}}$ & Radium ion & $\mathrm{Ra}^{2+}$ & 100 \\
\hline
\end{tabular}

Information from Brookins (1988).

Note: $E$ Eh $=300 \mathrm{mV}$ and $\mathrm{pH}=6.3$ (conditions observed in downgradient monitor well 414 , completed in the unconsolidated materials, during the January 1991 sampling round). 
precipitates could become an important mechanism for attenuation of manganese in the plume. Modeling with PHREEQE (Parkhurst et al., 1980) indicates that mixing with the higher $\mathrm{pH}$ water in the lower unit could more strongly stabilize $\mathrm{Fe}$ and $\mathrm{Mn}$ oxides and hydroxides in the aquifer matrix, and these metals would tend to be adsorbed or precipitated. An increase in pH accompanied by the high alkalinity of the bedrock water could result in precipitation of the mineral rhodochrosite $\left(\mathrm{MnCO}_{3}\right)$ and manganese attenuation.

\section{Molybdenum}

In samples taken since 1987, molybdenum occurs in concentrations from near the detection limit to $0.02 \mathrm{mg} / \mathrm{L}$ in background well 410 and from near the detection limit to $0.03 \mathrm{mg} / \mathrm{L}$ in downgradient well 414 (Figure 3.3). Samples taken in August 1986 are high for all wells sampled. Values reported for both background well 410 and downgradient well 414 are $0.20 \mathrm{mg} / \mathrm{L}$ during that sampling round. Modeling with PHREEQE (Parkhurst et al., 1980) indicates that molybdenum occurs in these waters as the molybdate species $\mathrm{MoO}_{4}{ }^{2-}$

(Table 3.4). This species becomes relatively mobile in water with $\mathrm{pH}$ values above 5 (Brookins, 1988). Thus, the primary mechanisms for decreasing molybdenum concentrations will be dilution and dispersion as ground water discharges into Chartiers Creek. Coprecipitation with and adsorption on iron oxides and hydroxides may be a secondary mechanism for removing molybdenum from ground water, particularly if contamination is transported into the bedrock unit.

\section{$\underline{\text { Uranium }}$}

Uranium concentrations of 0.01 to $0.04 \mathrm{mg} / \mathrm{L}$ in downgradient well 414 are significantly higher than those in background well 410 (below detection to $0.003 \mathrm{mg} / \mathrm{L}$ ) (Figure 3.4). Modeling with PHREEQE (Parkhurst et al., 1980) predicts that various uranyl carbonates are the dominant uranium species in solution (Table 3.4). These species are relatively stable in mildly oxidizing and alkaline solutions such as water in the upper ground water system at the Canonsburg site. Thus, the major reduction in uranium concentrations at the site will occur by dilution as ground water discharges into Chartiers Creek. Adsorption on iron and manganese oxides and hydroxides could also be important in removing uranium from the ground water. If uranium were to migrate from the unconsolidated unit to the bedrock system, more reducing conditions could stabilize the mineral uraninite in the system and uranium would be precipitated.

\section{Radium-226 and radium-228}

In data from 10 sampling dates for downgradient well 414 , radium-226 is above detection only on 28 October 1993, with a value of $0.7 \pm 0.5$ picocuries per liter $(\mathrm{pCi} / \mathrm{L})$ for the filtered sample. Background well 410 also shows values below detection except on 5 November 1986, which shows a value of $2.1 \pm 0.6 \mathrm{pCi} / \mathrm{L}$. Similarly, radium-228 is above detection in monitor well 414 
only once in 10 sampling dates with a value of $2.3 \pm 1.0 \mathrm{pCi} / \mathrm{L}$ on 1 August 1991 , and twice in monitor well 410 with a high value of $1.2 \pm 1.0$ on 1 August 1991. These values are not statistically different.

Radium is an alkaline-earth metal that shares many chemical characteristics with the more common alkaline-earth element barium. Like barium, radium forms a highly insoluble sulfate compound at $\mathrm{pH}$ values ranging from 3 to more than 12 (Brookins, 1988), but ion exchange reactions primarily are expected to control solution concentrations. Radium is more strongly adsorbed by clays and iron oxides/hydroxides than are strontium, calcium, and magnesium, suggesting that radium should be essentially immobile in ground water at the Canonsburg site.

\section{Lead-210}

Very little data are available for lead-210. Water from background well 410 was analyzed once in 1986 for lead-210 (filtered sample), with results reported as $1.1 \pm 1.1 \mathrm{pCi} / \mathrm{L}$, and once in 1993 (unfiltered sample), with results reported as $0.7 \pm 1.5 \mathrm{pCi} / \mathrm{L}$. For downgradient well 414, a single analysis in 1986 (filtered sample) shows $5.0 \pm 1.2 \mathrm{pCi} / \mathrm{L}$ and an analysis in 1993 (unfiltered sample) shows $1.3 \pm 1.5 \mathrm{pCi} / \mathrm{L}$. Thus, lead-210 in downgradient well 414 is higher than background for a single analysis in 1986.

Processes affecting lead-210 in the ground water at the Canonsburg site are the same as those affecting lead in general. Modeling by PHREEOE (Parkhurst, et al., 1980) predicts that the dominant species in ground water from well 414 will be various carbonate species (Table 3.4). This water is not saturated with the lead carbonate mineral cerrusite, so it is unlikely that precipitation will remove lead. However, iron oxides and hydroxides are stable in the ground water matrix and these phases strongly adsorb lead. Therefore, lead contamination probably will not extend beyond the confines of the site.

\section{Polonium-210}

With only three sample dates reported, Polonium-210 was not detected at downgradient well 414. With two sample dates reported, background well 410 shows one concentration below detection and one at $0.2 \pm 0.1 \mathrm{pCi} / \mathrm{L}$.

Polonium-210 is produced by the beta decay of lead-210 (half-life equals 22 years) through the intermediate short-lived daughter bismuth-210 (half-life equals 5.02 days). Polonium-210 has a half-life of 138 days and decays to stable lead-206. Because lead-210 has a much longer half-life than bismuth210 or polonium-210, the distribution of lead-210 primarily will control the distribution of polonium-210 at this site.

\section{Thorium-230}

As with lead-210, data are sparse for this constituent. Thorium-230 in downgradient well 414 is above detection for only one sampling date on which 
the values in the filtered sample $(0.7 \pm 0.3 \mathrm{pCi} / \mathrm{L})$ were found to be higher than those in the unfiltered sample $(0.5 \pm 0.3 \mathrm{pCi} / \mathrm{L})$. With only two sampling dates, background well 410 also has one sample with values that are above detection $(1.4 \pm 0.3 \mathrm{pCi} / \mathrm{L}$ filtered and $0.3 \pm 0.3 \mathrm{pCi} / L$ unfiltered). These values are higher than those in downgradient well 414.

Whatever the source of thorium-230 in these wells, the highly insoluble $\operatorname{Th}(\mathrm{OH})_{4}$ and $\mathrm{ThO}_{2}$ (Brookins, 1988; Langmuir and Herman, 1980) dominate the Eh and $\mathrm{pH}$ ranges of ground water at Canonsburg. Therefore, it is likely that the Eh and $\mathrm{pH}$ conditions at the Canonsburg site will effectively immobilize thorium-230.

\subsection{SURFACE WATER MONITORING}

Up to six rounds of water quality data are available from surface locations at Chartiers Creek. In addition, one round of sediment data is available from each location. Based on these data, there is no evidence that the site contributes contamination to the creek. Detailed discussion of these data is provided below.

Figure 3.5 shows the six sampling locations (601 through 606). Prior to the October 1993 ecological field survey, only locations 601 (upstream of the site) and 602 (adjacent to Area $C$ ) had been sampled. One to three filtered surface water samples were collected from these two locations during 1989 and 1990. One to four unfiltered surface water samples were collected from these locations from 1991 through 1993 (Table 3.5). Comparing the unfiltered water sample data from location 601 with location 602 indicates no statistically significant differences in water quality between the two sampling sites (DOE, 1995). Uranium was not detected $(<0.001 \mathrm{mg} / \mathrm{L})$ at either site. Five of the ground water constituents (ammonium, magnesium, manganese, sodium, and sulfate) were detected at the upstream location (601) at median concentrations equal to or greater than concentrations observed at the downstream location (602). For the other four constituents (calcium, chloride, molybdenum, and potassium), the concentrations detected at location 602 were higher than the upstream concentrations. However, the differences in concentrations between the two sites for these four constituents are minimal, ranging from 2 to 6 percent, and do not represent a statistically significant difference (DOE, 1995).

Surface water samples were collected in October 1993 at all sampling locations (601 through 606). Locations 603 through 606 were not sampled prior to this date. The samples collected in October 1993 were analyzed for a select list of constituents (chromium, iron, lead, manganese, molybdenum, selenium, sulfate, uranium, vanadium, and zinc). These constituents were chosen based on a preliminary screening of the ground water data prior to development of this risk assessment. Of these constituents, concentrations of manganese, molybdenum, sulfate, and uranium were identified as exceeding background levels in ground water. An evaluation of the most recent surface water data (October 1993) for all sampling locations (see Section 7.0) indicates that uranium was not detected at any location, and sulfate was detected at the 

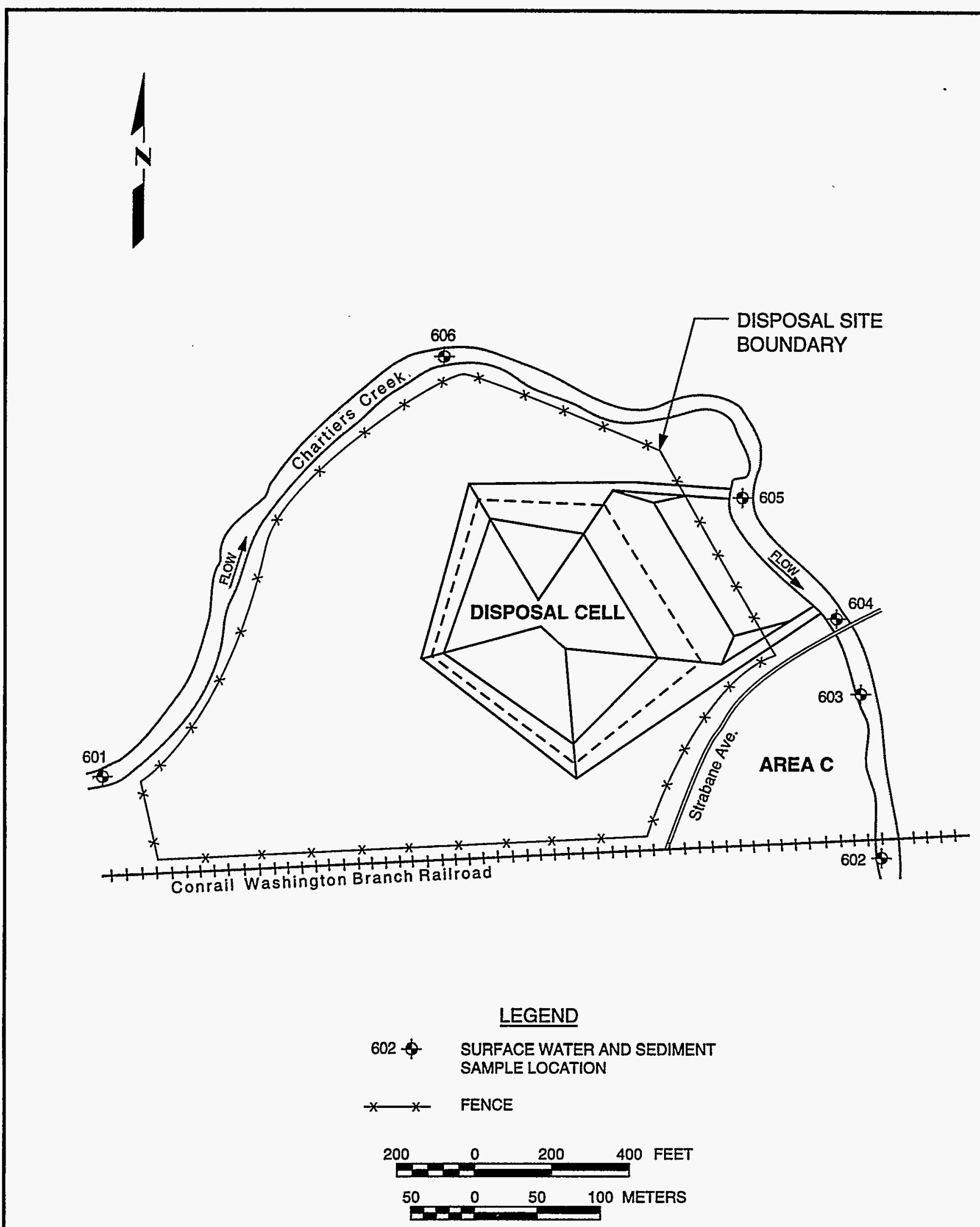

FIGURE 3.5

SURFACE WATER AND SEDIMENT SAMPLE LOCATIONS CANONSBURG, PENNSYLVANIA, SITE 
Table 3.5 Comparison of upstream and downstream water quality data from Chartiers Creek, Canonsburg, Pennsylvania, site (1989-1993, unfiltered water samples)

\begin{tabular}{|c|c|c|c|c|}
\hline Parameter $^{a}$ & $\begin{array}{c}\text { Above } \\
\text { detection }\end{array}$ & Minimum & Median' & Maximum \\
\hline Inorganic para & & & $\mathrm{mg} / \mathrm{L}$ & \\
\hline $\begin{array}{l}\text { Ammonium } \\
\text { Upstream } \\
602\end{array}$ & $\begin{array}{l}3 / 4 \\
3 / 4\end{array}$ & $\begin{array}{l}<0.06 \\
<0.06\end{array}$ & $\begin{array}{l}0.25 \\
0.20\end{array}$ & $\begin{array}{l}0.8 \\
0.3\end{array}$ \\
\hline $\begin{array}{l}\text { Cadmium } \\
\text { Upstream } \\
602\end{array}$ & $\begin{array}{l}0 / 1 \\
0 / 1\end{array}$ & - & $\begin{array}{l}<0.001 \\
<0.001\end{array}$ & - \\
\hline $\begin{array}{l}\text { Calcium } \\
\text { Upstream } \\
602\end{array}$ & $\begin{array}{l}5 / 5 \\
5 / 5\end{array}$ & $\begin{array}{l}80.1 \\
80.0\end{array}$ & $\begin{array}{l}83.0 \\
86.7\end{array}$ & $\begin{array}{l}112 \\
116\end{array}$ \\
\hline $\begin{array}{l}\text { Chloride } \\
\text { Upstream } \\
602\end{array}$ & $\begin{array}{l}5 / 5 \\
5 / 5\end{array}$ & $\begin{array}{l}36 \\
34\end{array}$ & $\begin{array}{l}50.1 \\
50.8\end{array}$ & $\begin{array}{r}99 \\
101\end{array}$ \\
\hline $\begin{array}{l}\text { Chromium } \\
\text { Upstream } \\
602 \\
\text { Downstream }^{\dagger}\end{array}$ & $\begin{array}{l}0 / 2 \\
0 / 2 \\
0 / 4\end{array}$ & $\begin{array}{l}<0.01 \\
<0.01 \\
<0.01\end{array}$ & - & $\begin{array}{l}<0.01 \\
<0.01 \\
<0.01\end{array}$ \\
\hline $\begin{array}{l}\text { Cobalt } \\
\text { Upstream } \\
602\end{array}$ & $\begin{array}{l}0 / 1 \\
0 / 1\end{array}$ & - & $\begin{array}{l}<0.05 \\
<0.05\end{array}$ & - \\
\hline $\begin{array}{l}\frac{\text { Cyanide }}{\text { Upstream }} \\
602\end{array}$ & $\begin{array}{l}1 / 4 \\
0 / 4\end{array}$ & $\begin{array}{l}<0.01 \\
<0.01\end{array}$ & - & $\begin{array}{r}0.02 \\
<0.01\end{array}$ \\
\hline $\begin{array}{l}\frac{\text { Iron }}{\text { Upstream }} \\
602 \\
\text { Downstream }\end{array}$ & $\begin{array}{l}6 / 6 \\
6 / 6 \\
4 / 4\end{array}$ & $\begin{array}{l}0.36 \\
0.36 \\
0.57\end{array}$ & $\begin{array}{l}0.565 \\
0.520 \\
0.650\end{array}$ & $\begin{array}{l}2.80 \\
2.92 \\
0.74\end{array}$ \\
\hline $\begin{array}{l}\frac{\text { Lead }}{\text { Upstream }} \\
602 \\
\text { Downstream }\end{array}$ & $\begin{array}{l}2 / 6 \\
3 / 6 \\
2 / 4\end{array}$ & $\begin{array}{c}<0.0015 \\
0.0022 \\
<0.003\end{array}$ & - & $\begin{array}{l}0.009 \\
0.012 \\
0.004\end{array}$ \\
\hline $\begin{array}{l}\text { Magnesium } \\
\text { Upstream } \\
602\end{array}$ & $\begin{array}{l}5 / 5 \\
5 / 5\end{array}$ & $\begin{array}{l}16.9 \\
16.8\end{array}$ & $\begin{array}{l}18.8 \\
18.9\end{array}$ & $\begin{array}{l}29.2 \\
29.5\end{array}$ \\
\hline $\begin{array}{l}\text { Manganese } \\
\text { Upstream } \\
602 \\
\text { Downstream }\end{array}$ & $\begin{array}{l}6 / 6 \\
6 / 6 \\
4 / 4\end{array}$ & $\begin{array}{l}0.13 \\
0.12 \\
0.12\end{array}$ & $\begin{array}{l}0.155 \\
0.155 \\
0.140\end{array}$ & $\begin{array}{l}0.20 \\
0.22 \\
0.17\end{array}$ \\
\hline
\end{tabular}


Table 3.5 Comparison of upstream and downstream water quality data from Chartiers Creek, Canonsburg, Pennsylvania, site (1989-1993, unfiltered water samples) (Continued)

\begin{tabular}{|c|c|c|c|c|}
\hline Parameter $^{a}$ & $\begin{array}{c}\text { Above } \\
\text { detection }^{b}\end{array}$ & Minimum & Medianc & Maximum \\
\hline & & & mg/L & \\
\hline $\begin{array}{l}\text { Molybdenum } \\
\text { Upstream } \\
602 \\
\text { Downstream }\end{array}$ & $\begin{array}{l}6 / 6 \\
6 / 6 \\
4 / 4\end{array}$ & $\begin{array}{l}0.04 \\
0.04 \\
0.10\end{array}$ & $\begin{array}{l}0.080 \\
0.085 \\
0.125\end{array}$ & $\begin{array}{l}0.22 \\
0.17 \\
0.17\end{array}$ \\
\hline $\begin{array}{l}\text { Nitrate } \\
\text { Upstream } \\
602\end{array}$ & $\begin{array}{l}5 / 5 \\
5 / 5\end{array}$ & $\begin{array}{l}0.43 \\
0.43\end{array}$ & $\begin{array}{l}11.7 \\
11.9\end{array}$ & $\begin{array}{l}31.0 \\
26.6\end{array}$ \\
\hline $\begin{array}{l}\text { Potassium } \\
\text { Upstream } \\
602\end{array}$ & $\begin{array}{l}5 / 5 \\
5 / 5\end{array}$ & $\begin{array}{l}3.6 \\
3.3\end{array}$ & $\begin{array}{l}6.5 \\
6.8\end{array}$ & $\begin{array}{l}10.4 \\
10.2\end{array}$ \\
\hline $\begin{array}{l}\text { Selenium } \\
\text { Upstream } \\
602 \\
\text { Downstream }\end{array}$ & $\begin{array}{l}0 / 6 \\
0 / 6 \\
0 / 4\end{array}$ & $\begin{array}{l}<0.0015 \\
<0.0015 \\
<0.005\end{array}$ & - & $\begin{array}{l}<0.05 \\
<0.005 \\
<0.005\end{array}$ \\
\hline $\begin{array}{l}\text { Sodium } \\
\text { Upstream } \\
602\end{array}$ & $\begin{array}{l}5 / 5 \\
5 / 5\end{array}$ & $\begin{array}{l}36.7 \\
36.0\end{array}$ & $\begin{array}{l}62 \\
58\end{array}$ & $\begin{array}{l}132 \\
135\end{array}$ \\
\hline $\begin{array}{l}\text { Sulfate } \\
\text { Upstream } \\
602 \\
\text { Downstream }\end{array}$ & $\begin{array}{l}6 / 6 \\
6 / 6 \\
4 / 4\end{array}$ & $\begin{array}{l}116 \\
128 \\
248\end{array}$ & $\begin{array}{l}207.0 \\
202.5 \\
262.0\end{array}$ & $\begin{array}{l}383 \\
412 \\
276\end{array}$ \\
\hline $\begin{array}{l}\text { Thallium } \\
\text { Upstream } \\
602\end{array}$ & $\begin{array}{l}0 / 3 \\
0 / 3\end{array}$ & $\begin{array}{l}<0.01 \\
<0.01\end{array}$ & - & $\begin{array}{l}<0.01 \\
<0.01\end{array}$ \\
\hline $\begin{array}{l}\text { Uranium } \\
\text { Upstream } \\
602 \\
\text { Downstream }\end{array}$ & $\begin{array}{l}1 / 5 \\
1 / 5 \\
0 / 4\end{array}$ & $\begin{array}{l}<0.001 \\
<0.001 \\
<0.001\end{array}$ & $\begin{array}{l}- \\
- \\
-\end{array}$ & $\begin{array}{r}0.001 \\
0.002 \\
<0.001\end{array}$ \\
\hline $\begin{array}{l}\text { Vanadium } \\
\text { Upstream } \\
602 \\
\text { Downstream }\end{array}$ & $\begin{array}{l}2 / 6 \\
2 / 6 \\
4 / 4\end{array}$ & $\begin{array}{c}<0.0019 \\
<0.0019 \\
0.01\end{array}$ & $\begin{array}{l}- \\
- \\
0.025\end{array}$ & $\begin{array}{l}0.02 \\
0.02 \\
0.03\end{array}$ \\
\hline $\begin{array}{l}\text { Zinc } \\
\text { Upstream } \\
602 \\
\text { Downstream }\end{array}$ & $\begin{array}{l}1 / 1 \\
1 / 1 \\
4 / 4\end{array}$ & $\begin{array}{l}- \\
- \\
0.040\end{array}$ & $\begin{array}{l}0.045 \\
0.199 \\
0.047\end{array}$ & $\begin{array}{l}- \\
- \\
0.064\end{array}$ \\
\hline
\end{tabular}


Table 3.5 Comparison of upstream and downstream water quality data from Chartiers Creek, Canonsburg, Pennsylvania, site (1989-1993, unfiltered water samples) (Concluded)

\begin{tabular}{|c|c|c|c|c|}
\hline Parameter ${ }^{\circ}$ & $\begin{array}{c}\text { Above } \\
\text { detection }\end{array}$ & Minimum & Median" & Maximum \\
\hline \multicolumn{2}{|c|}{ Radiological parameters } & \multicolumn{3}{|c|}{ pCi/L } \\
\hline \multicolumn{5}{|l|}{ Radium-226 } \\
\hline $\begin{array}{l}\text { Upstream } \\
602\end{array}$ & $\begin{array}{l}4 / 4 \\
4 / 4\end{array}$ & $\begin{array}{l}\text { ND } \\
\text { ND }\end{array}$ & $\begin{array}{l}0.12 \\
0.0\end{array}$ & $\begin{array}{l}0.90 \\
0.12\end{array}$ \\
\hline
\end{tabular}

aData were available from sampling events as early as 1989. All sampling events were used in this summary.

${ }^{b}$ Above detection $=$ Number of samples with detectable concentration/total number of samples.

Median is the $50^{\text {th }}$ percentile of the data. The median cannot be calculated if 50 percent or more of the data are below detection. For parameters for which only one sampling round of data was available, the one reported value is listed in the median column.

dUpstream sampling location 601 was used as background. Sampling data from 1989 through 1993 were available for this location.

'Sampling location 602 was the only downstream location for which sufficient historical data were available for statistical evaluation.

'Sampling locations 603, 604, 605, and 606 were also considered to be downstream. These locations were sampled only during the October 1993 sampling round, and these samples were analyzed for only 10 inorganic parameters. 
highest concentration at the upstream location (Table 3.5). For the other two constituents (manganese and molybdenum), the differences between the upstream concentrations and adjacent or downstream concentrations ranged from 10 to 47 percent, with the highest values detected at location 606 . Although the surface water quality data base is limited, the available data suggest that ground water contamination from the site is not affecting the water quality of Chartiers Creek. However, acid mine drainage (the closest of several upstream mines is approximately $1 \mathrm{mi}[1.6 \mathrm{~km}]$ southwest of the site) and industrial and municipal discharges (FBDU, 1982; Newport, 1973) affect water quality in Chartiers Creek. Concentrations of sulfate, iron manganese, and TDS are elevated in surface water at both upstream and downstream sampling locations (Table 3.5).

Sediment samples were collected at all surface water sampling locations 1601 through 606) in October 1993. Table 3.6 is a summary of the sediment sampling data. Sediments are an integral part of the aquatic environment, providing habitat, feeding, and rearing areas for many aquatic organisms (Hull and Suter, 1994). The sediment layer includes solid particles (inorganic and organic) settling on the bottom of a body of water such as a river or a pond, and interstitial water (pore water), which fills the spaces between the sediment particles (Power and Chapman, 1992). In the environment, these particles are derived both from material originally suspended in the water and minerals that precipitate from the water. Pore water usually accounts for over 50 percent (by volume) of the sediment layer. Note that results presented in this risk assessment represent the chemistry of both the solid and pore-water components of the sediment layer. Potentially, site-related ground water could affect sediment concentrations of certain constituents. However, information available from only one sampling round does not allow assessment of this potential. If site-related constituents were to accumulate in sediment they could then act as a source of site-related surface water contamination. However, as discussed in Section 7.0, no evidence suggests that this is now occurring. 
Table 3.6 Comparison of upstream and downstream sediment data from Chartiers Creek, Canonsburg, Pennsylvania, site (1993)

\begin{tabular}{|c|c|c|c|c|}
\hline Parameter $^{\circ}$ & $\begin{array}{c}\text { Above } \\
\text { detection }^{b}\end{array}$ & Minimum & Median ${ }^{c}$ & Maximum \\
\hline Inorganic parameters & & & $\mathrm{mg} / \mathrm{L}$ & \\
\hline $\begin{array}{l}\text { Chromium } \\
\text { Upstream }^{d} \\
\text { Downstream }^{e}\end{array}$ & $\begin{array}{l}1 / 1 \\
5 / 5\end{array}$ & 22 & $\begin{array}{l}96 \\
69\end{array}$ & 189 \\
\hline $\begin{array}{l}\frac{\text { Iron }}{\text { Upstream }} \\
\text { Downstream }\end{array}$ & $\begin{array}{l}1 / 1 \\
5 / 5\end{array}$ & $26,200^{-}$ & $\begin{array}{l}37,400 \\
56,600\end{array}$ & $105,000^{-}$ \\
\hline $\begin{array}{l}\text { Lead } \\
\text { Upstream } \\
\text { Downstream }\end{array}$ & $\begin{array}{l}1 / 1 \\
5 / 5\end{array}$ & 0.42 & $\begin{array}{l}80.8 \\
49.5\end{array}$ & 53.7 \\
\hline $\begin{array}{l}\text { Manganese } \\
\text { Upstream } \\
\text { Downstream }\end{array}$ & $\begin{array}{l}1 / 1 \\
5 / 5\end{array}$ & $590^{-}$ & $\begin{array}{l}1,410 \\
1,900\end{array}$ & $2,820^{-}$ \\
\hline $\begin{array}{l}\text { Molybdenum } \\
\text { Upstream } \\
\text { Downstream }\end{array}$ & $\begin{array}{l}1 / 1 \\
5 / 5\end{array}$ & - & $\begin{array}{l}33 \\
16\end{array}$ & 96 \\
\hline $\begin{array}{l}\text { Selenium } \\
\text { Upstream } \\
\text { Downstream }\end{array}$ & $\begin{array}{l}0 / 1 \\
1 / 5\end{array}$ & $<0.5$ & $\begin{array}{l}<0.5 \\
<0.5\end{array}$ & - \\
\hline $\begin{array}{l}\text { Uranium } \\
\text { Upstream } \\
\text { Downstream }\end{array}$ & $\begin{array}{l}1 / 1 \\
5 / 5\end{array}$ & $\overline{1.6}$ & $\begin{array}{l}2.0 \\
2.0\end{array}$ & 2.6 \\
\hline $\begin{array}{l}\text { Vanadium } \\
\text { Upstream } \\
\text { Downstream }\end{array}$ & $\begin{array}{l}1 / 1 \\
5 / 5\end{array}$ & $2 \overline{2}$ & $\begin{array}{l}42 \\
41\end{array}$ & 126 \\
\hline $\begin{array}{l}\frac{\text { Zinc }}{\text { Upstream }} \\
\text { Downstream }\end{array}$ & $\begin{array}{l}1 / 1 \\
5 / 5\end{array}$ & 90 & $\begin{array}{l}252 \\
204\end{array}$ & $268^{-}$ \\
\hline
\end{tabular}

${ }^{\mathrm{a} D a t a}$ were available from a single sampling round in October 1993.

${ }^{b}$ Above detection $=$ Number of samples with detectable concentration/total number of samples.

'Median is the $50^{\text {th }}$ percentile of the data. The median cannot be calculated if 50 percent or more of the data are below detection. When data were available from only one sampling round, the single value is reported in the median column.

'Upstream sampling location 601 was used as background.

Sampling locations 602, 603, 604, 605, and 606 were combined as downstream. 


\subsection{EXPOSURE ASSESSMENT}

This section discusses and estimates the type and magnitude of exposures to the chemicals of potential concern present at the Canonsburg site. The exposure pathways evaluated in this section are based on potential contact with ground water, surface water, and sediment at the site or in the site vicinity.

\subsection{POTENTIALLY EXPOSED POPULATION}

Ground water contaminated by uranium processing at the Canonsburg site is not currently used. The source of drinking water for residents in the site vicinity is the Monongahela River as supplied by the Pennsylvania-American Water Company. As shown in Figure 2.10, a number of residents within a 1-mi $(1.6-\mathrm{km})$ site radius have domestic wells; however, most of these wells are hydrologically upgradient or crossgradient from the site and they are not in use. Additionally, in the future, it is unlikely that residents will ever use these wells for domestic purposes. The shallow ground water is not considered as a potable water resource in the Canonsburg site area, and good quality water is available from the municipal water supply system. Residents located slightly beyond $1 \mathrm{mi}$ $(1.6 \mathrm{~km})$ north and south of the site use the ground water for drinking purposes, and one well approximately $400 \mathrm{ft}(120 \mathrm{~m})$ north of the site is used for domestic purposes other than drinking. However, contaminated ground water from the site appears to be confined within the Canonsburg site area. Chartiers Creek probably creates a barrier for the contamination. Therefore, sitecontaminated ground water has not migrated to off-site locations and is not expected to affect these residents (see Sections 2.4 and 2.7).

Because there are no current human receptors of contaminated ground water, this assessment assumes a future resident ground water use scenario. In the future, a domestic well could be installed in the unconsolidated material in Area $C$, creating the potential for exposure through drinking, bathing, and irrigation. This assumption is made because Area $C$ is the only portion of the site available for future public use. However, the likelihood for residential development at the site is considered low. The exposure point in this area will be based on data from well 414. Although wells from other areas of the site (i.e., well 412 and 413) have higher levels of some contaminants, data from these wells were not used in this assessment because no one will use the ground water in this area. This portion of the site will be in DOE possession, and development of this area will not be permitted (TAC, 1994b). The ground water in this area discharges to Chartiers Creek. Based on available data, it appears that contamination has not migrated to the bedrock (see Section 2.4).

The future resident scenario evaluates domestic ground water uses consistent with current water uses by the population in the region. The potentially exposed population includes residents of the following age groups: children ( 1 to 10 years old and 6 to 12 years old) and adults (11 to 65 years old). These age groups were selected for the following reasons: 
- Survey data for population variables such as age, weight, and daily water intake are available for these age groups.

- Toxicological variables are similar within these age groups, including responsiveness of sensitive subgroups to the contaminants of concern, consistent intake to body weight ratios, and similar toxicokinetics (a study of the time course of absorption, distribution, metabolism, and excretion of a contaminant in an individual's body).

- Potential exposures to nearby populations may occur in surface waters near the site because ground water in the unconsolidated material at the site discharges into Chartiers Creek. Local residents (adults and older children) could use these waters for wading, swimming, or fishing.

\subsection{EXPOSURE PATHWAYS}

An exposure pathway describes the course a chemical takes from a source to an exposed organism. Exposure can occur only if there is a source of contamination, a point of contact with a population or individual, and a route of exposure (e.g., water ingestion). Because the tailings piles and soils contaminated from uranium operations at the site were removed and relocated to a disposal cell, soil or air exposure pathways (such as accidental soil ingestion, dermal contact with soil, or inhalation of particulates) are not considered. This assessment evaluates both ground water and surface water/sediment pathways. Figure 4.1 presents a conceptual model of the potential ground water and surface water/sediment exposure pathways that may occur at the Canonsburg site. The following subsections discuss these pathways in more detail.

\subsubsection{Ground water}

Area $\mathrm{C}$ is the only portion of the site available for future development. As discussed earlier in this document, the majority of the site (the disposal site area-see Figure $\mathbf{2 . 2}$ for location), is within a permanently restricted zone. Therefore, only ground water beneath Area $C$ is evaluated for possible future use.

Although it is unlikely that ground water in the site vicinity will be used in the future for drinking purposes because of the existing public water supply system, this risk assessment will assess hypothetical future use of the ground water in the unconsolidated material. Water in the region is primarily used for household purposes such as drinking, cooking, and bathing. Another use typical of the region that could indirectly lead to human exposure is irrigation of garden vegetables. The conceptual model (Figure 4.1 ) presents the potential ground water exposure routes that could result from these uses and includes drinking water ingestion, dermal absorption, and garden produce ingestion. 


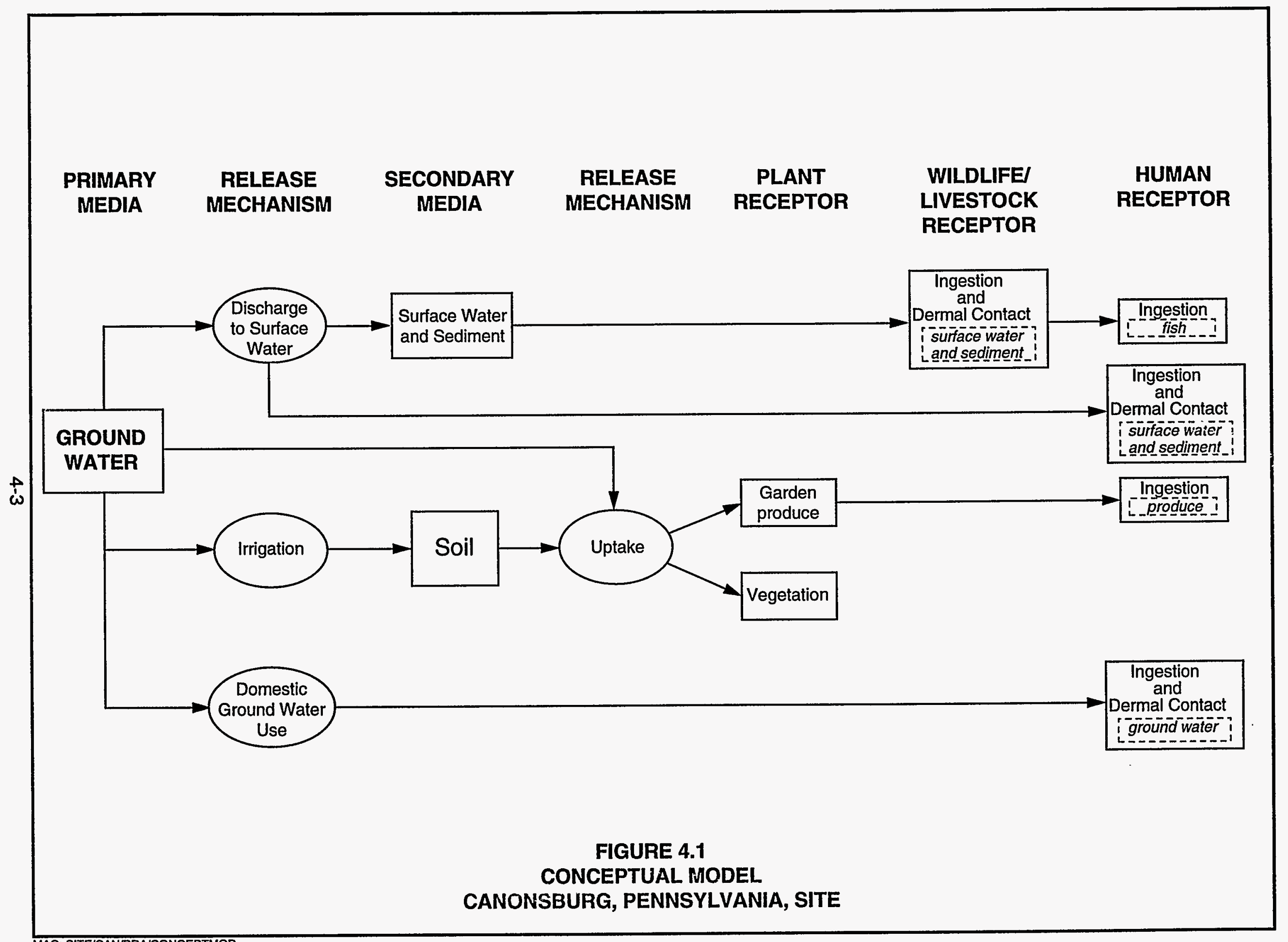




\section{Drinking water ingestion}

Drinking water ingestion is generally the dominant exposure route for ground water contaminated with metals (DOE, 1994). For this evaluation, drinking water consumption includes water used for drinking and for food preparation (e.g., reconstituted juice, soup, rice, and beans). For comparing relative pathway significance, Table 4.1 presents a screening level assessment of drinking water intake for adults. These screening calculations are based on the estimated 95 th percentile of the constituent concentrations measured at well 414.

\section{Dermal absorption}

Dermal absorption is the process by which chemicals coming into contact with the skin are absorbed into the blood vessels near the skin's surface. Some compounds are absorbed easily, although metals do not possess the chemical properties conducive to skin absorption.

To evaluate this exposure pathway, a screening calculation was performed to determine if the dermal absorption dose would be substantial compared with the drinking water ingestion dose for the contaminants of potential concern. Because chemical-specific absorption factors are not available for the contaminants of potential concern, they are assumed to be absorbed across the skin at the same rate as water. This assumption will probably overestimate any potential contribution from dermal absorption.

Table 4.1 provides the results of the screening. Although the dermal dose is an absorbed dose-whereas the drinking water ingestion dose is a total dose of which only some percentage will be absorbed-the very low $(0.2$ percent) contribution of dermal absorption is assumed to be insignificant compared with drinking water ingestion. Based on these results, the dermal absorption exposure route is eliminated from a more detailed evaluation.

\section{Ingestion of ground water-irrigated garden produce}

The garden produce ingestion pathway could not be evaluated. Although the intake from ground water-irrigated produce ingestion is not likely to be greater than the exposure dose from drinking water ingestion, the incremental contribution could be substantial. There are currently no literature values that could be used to estimate this pathway contribution. However, the UMTRA Ground Water Project is currently conducting plant uptake studies for irrigated vegetables and grasses. The results of these studies will be included in the National Environmental Policy Act (NEPA) document and ground water compliance strategy planning for this site. 
Table 4.1 Exposure dose calculations and equation definitions for ground water ingestion and dermal contact for the future hypothetical adult scenario, Canonsburg, Pennsylvania, site

\begin{tabular}{|c|c|c|c|c|}
\hline \multirow{2}{*}{$\begin{array}{c}\text { Contaminant of } \\
\text { potential } \\
\text { concern }\end{array}$} & \multirow[b]{2}{*}{ Cw } & \multicolumn{2}{|c|}{$\begin{array}{l}\text { Ground water exposure doses } \\
\text { (mg/kg-day) }\end{array}$} & \multirow{2}{*}{$\begin{array}{c}\text { Ratio of } \\
\text { dermal: } \\
\text { ingestion }\end{array}$} \\
\hline & & Ingestion & Dermal contact & \\
\hline \multicolumn{5}{|c|}{ Noncarcinogenic effects (mg/L) } \\
\hline Manganese & 10 & $3 E-01$ & $5 E-04$ & 0.002 \\
\hline Molybdenum & 0.027 & 7E-04 & 1E-06 & 0.002 \\
\hline Uranium & 0.033 & 9E-04 & $2 E-06$ & 0.002 \\
\hline \multicolumn{5}{|c|}{ Carcinogenic effects (pCi/L) } \\
\hline Uranium & $23^{b}$ & $5 E+05^{c}$ & $9 E+02^{c}$ & 0.002 \\
\hline
\end{tabular}

Equation definitions for exposure dose calculations

Ingestion of ground water

Chemicals: $\quad$ Chronic daily intake $\left(\mathrm{mg} / \mathrm{kg}\right.$-day) $=\frac{\mathrm{Cw} \times \operatorname{IRw} \times \mathrm{EF} \times \mathrm{ED}}{\mathrm{BW} \times \mathrm{AT}}$

Radionuclides: Lifetime intake ( $\mathrm{pCi}$ per lifetime) $=\mathrm{Cw} \times \mathrm{IRw} \times \mathrm{EF} \times \mathrm{ED}$

Dermal contact with ground water

Chemicals: Chronic daily intake $(\mathrm{mg} / \mathrm{kg}$-day $)=\frac{(\mathrm{Cw} \times \mathrm{SA} \times \mathrm{Pc} \times \mathrm{Cf}) \times \mathrm{ET} \times \mathrm{EF} \times \mathrm{ED}}{\mathrm{BW} \times \mathrm{AT}}$

Radionuclides: Lifetime intake ( $p$ Ci per lifetime) $=\mathrm{Cw} \times \mathrm{SA} \times \mathrm{Pc} \times \mathrm{Cf} \times \mathrm{ET} \times \mathrm{EF} \times \mathrm{ED}$

\section{Where:}

$\mathrm{CW}=$ Contaminant concentration in ground water (upper 95th percentile) $(\mathrm{mg} / \mathrm{L}$ or $\mathrm{pCi} / \mathrm{L})$.

IRw = Ingestion rate for water ( $L$ per day) ( $2 \mathrm{~L}$ per day for an adult) (EPA, 1989a).

$E F=$ Exposure frequency (350 days per year) (EPA, 1991).

$E D=$ Exposure duration (30 years for an adult) (EPA, 1989a).

$B W=$ Body weight (70 kilograms [kg] for an adult) (EPA, 1989a).

AT = Averaging time (365 days $\times$ ED for noncarcinogens) (EPA, 1989a).

$\mathrm{SA}=$ Skin surface area $\left(19,400 \mathrm{~cm}^{2}\right.$; based on 50 th percentile total body surface area) (EPA, 1989a).

$\mathrm{Pc}=$ Dermal permeability constant $(0.001 \mathrm{~cm}$ per hour) (EPA, 1989a).

Cf = Conversion factor $\left(0.001\right.$ liters per cubic centimeter $\left.\left[\mathrm{L} / \mathrm{cm}^{3}\right]\right)$.

ET = Exposure time (0.2 hour per day) (EPA, 1989a).

"Ratio of the dermal absorption exposure dose to the ground water ingestion dose.

'Uranium-234 and uranium-238 combined; $1 \mathrm{mg}$ uranium is assumed to equal $686 \mathrm{pCi}$.

'Units are $\mathrm{pCi}$ per lifetime.

$\mathrm{mg} / \mathrm{kg}$-day $=$ milligrams per kilogram per day. 


\section{Summary}

The results of the ground water pathway screening analyses indicate that drinking water ingestion is the dominant pathway (see also DOE, 1994). Section 4.4 further evaluates this pathway probabilistically.

\subsubsection{Surface water/sediment}

The surface water/sediment exposure pathway is considered due to the potential access of nearby residents to Chartiers Creek, which receives ground water discharge from the unconsolidated material beneath the site. Individuals have access to the creek for swimming or wading in the summer. The creek also is used for fishing. For these reasons, the surface water and sediment in the creek were selected for a detailed exposure evaluation. The conceptual model (Figure 4.1) presents the potential surface water and sediment exposure pathways that could result, and includes incidental surface water ingestion, dermal absorption from surface water, incidental sediment ingestion, and fish ingestion. Children aged 6 to 12 years are considered the subpopulation most likely to play in the creek, and adults are considered the subpopulation most likely to fish and subsequently consume their catch.

\section{Incidental ingestion of surface water}

Incidental surface water ingestion could occur when children swim or wade in Chartiers Creek. It is assumed that a child may visit the creek every day during the summer to swim or wade, incidentally ingesting a few tablespoons of water on each visit. Table 4.2 presents the results for this exposure pathway, which is further evaluated in Section 6.0.

\section{Dermal contact with surface water}

The dermal contact exposure route for surface water was evaluated for the same scenario discussed under incidental ingestion of surface water. A child's total body surface area is assumed to contact surface water while swimming in the creek. Dermal exposure was evaluated for swimming rather than wading because more skin surface area would contact water while swimming. Dermal contact with surface water leads to a potential exposure route from dermal absorption, although metals generally do not absorb well across the skin. Because chemical-specific absorption factors are not available for the contaminants of concern, they were assumed to absorb across the skin at the same rate as water. This assumption probably overestimates any potential contribution from dermal absorption. Table 4.2 presents the results for this exposure route showing that contributions from dermal absorption are approximately 20 percent of the incidental surface water ingestion dose for the selected contaminants of concern. Based on these results, the dermal absorption from the surface water pathway is further evaluated in Section 6.0. 
Table 4.2 Exposure dose calculations and equation definitions for incidental surface water ingestion and dermal contact by children in Chartiers Creek, Canonsburg, Pennsylvania, site

\begin{tabular}{|c|c|c|c|c|}
\hline \multirow{2}{*}{$\begin{array}{l}\text { Contaminant of } \\
\text { potential concern }\end{array}$} & \multirow{2}{*}{$\begin{array}{c}\text { Csw } \\
(\mathrm{mg} / \mathrm{L})\end{array}$} & \multicolumn{2}{|c|}{$\begin{array}{l}\text { Surface water exposure doses } \\
\text { ( } \mathrm{mg} / \mathrm{kg} \text {-day) }\end{array}$} & \multirow{2}{*}{$\begin{array}{c}\text { Ratio of } \\
\text { dermal: } \\
\text { surface water } \\
\text { ingestion }\end{array}$} \\
\hline & & Ingestion & Dermal contact & \\
\hline $\begin{array}{l}\text { Manganese } \\
\text { Molybdenum } \\
\text { Uranium }\end{array}$ & $\begin{array}{l}0.17^{b} \\
0.17^{b} \\
N D\end{array}$ & $\begin{array}{l}5 \mathrm{E}-05 \\
5 \mathrm{E}-05 \\
\text { NA }\end{array}$ & $\begin{array}{c}1 E-05 \\
1 E-05 \\
N A\end{array}$ & $\begin{array}{l}0.2 \\
0.2 \\
\text { NA }\end{array}$ \\
\hline
\end{tabular}

\section{Equation definitions for exposure dose calculations}

Incidental ingestion of surface water

Chronic daily intake $\left(\mathrm{mg} / \mathrm{kg}\right.$-day) $=\frac{\text { Csw } \times \text { IRsw } \times \text { EF } \times \text { ED }}{B W \times A T}$

Dermal contact with surface water

Chronic daily intake $\left(\mathrm{mg} / \mathrm{kg}\right.$-day) $=\frac{(\text { Csw } \times \mathrm{SA} \times \mathrm{Pc} \times \mathrm{Cf}) \times \mathrm{ET} \times \mathrm{EF} \times \mathrm{ED}}{\mathrm{BW} \times \mathrm{AT}}$

Where:

Csw = Contaminant concentration in surface water (maximum concentration) (mg/L).

IRsw = Incidental ingestion rate for surface water $(0.05 \mathrm{~L} /$ day for 6 - to 12 -year-old children $)$ (EPA, 1989a).

$\mathrm{EF} \quad=$ Exposure frequency (90 days; 3 months per year for 7 days per week).

ED = Exposure duration ( 7 years for 6 to 12 year old children).

$\mathrm{BW}=$ Body weight $(38.3 \mathrm{~kg}$; based on 90 th percentile body weight for 6 - to 12-year-old male children) (EPA, 1989b).

AT = Averaging time (365 days $\times$ ED) (EPA, 1989a).

$\mathrm{SA}=$ Skin surface area $110,937 \mathrm{~cm}^{2}$ for 6 - to 12 -year-old children; based on 50 th percentile total body surface area) (EPA, 1989b).

$\mathrm{Pc}=$ Dermal permeability constant $(0.001 \mathrm{~cm}$ per hour) (EPA, 1989a).

Cf = Conversion factor $\left(0.001\right.$ liters per cubic centimeter $\left.\left[\mathrm{L} / \mathrm{cm}^{3}\right]\right)$.

ET = Exposure time (1 hour per day).

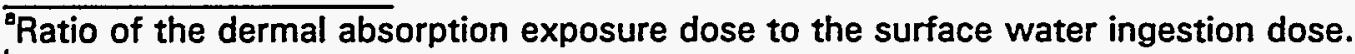

based on maximum detected value in surface water (sampling location 606).

ND - not detected in surface water.

$\mathrm{mg} / \mathrm{kg}$-day $=$ milligrams per kilogram per day.
} 


\section{Incidental ingestion of sediments}

Children swimming or wading in Chartiers Creek may also be exposed to contaminants of potential concern through the incidental ingestion of sediments. Table 4.3 presents the equation and assumptions for this exposure route. This exposure pathway could not be evaluated for potential carcinogenicity from radioactive uranium due to the lack of monitoring data of uranium radioisotopes in Chartiers Creek sediment. The doses from this exposure pathway exceed those estimated for incidental ingestion of surface water. Section 6.0 further evaluates the incidental ingestion of sediments exposure route.

\section{Fish ingestion}

The commonwealth of Pennsylvania designated Chartiers Creek for the maintenance and propagation of fish species and protection of additional flora and fauna indigenous to a warm water habitat (PADER, 1992). Fish found in the creek include carp, catfish, and bluegill (Templeton, 1993). The ingestion of fish, which may have accumulated site-related constituents, is a potential exposure pathway for residents who use the creek for fishing.

Table 4.4 presents the results of the screening-level evaluation for the fish ingestion exposure pathway. To evaluate this exposure route, chemical-specific bioconcentration factors (BCF) are needed to estimate fish tissue levels. A BCF for fish was not available in the literature for molybdenum. For manganese, assuming a BCF of 150 for fish, an intake rate higher than that estimated for the surface water ingestion route was calculated. Based on these results, the fish ingestion exposure pathway is further evaluated in Section 6.0.

\subsection{EXPOSURE CONCENTRATIONS}

The exposure concentration of a contaminant in ground water is defined as the concentration contacted by an individual over the period of exposure being considered. Estimates of contaminant concentrations are based on ground water data collected from monitoring wells after surface remediation was completed at the site. In this evaluation, the contaminant concentrations are assumed to be at steady state although actual contaminant concentrations land therefore potential exposures) are expected to decrease with time because surface remediation has been completed at the site. Nonetheless, these estimates are reasonable for chronic exposure soon after surface remediation. (Chronic exposure for noncarcinogens is considered to be exposure for any period longer than 7 years).

For the unconsolidated material, exposure concentrations are evaluated as a probability of occurrence based on 1988 to 1993 ground water quality data from monitor well 414. The probability distribution selected for each contaminant reflected, to the extent possible, the same mean, standard deviation, and shape observed in actual water quality data. For constituents showing an obvious trend over time, the theoretical distribution is centered 
Table 4.3 Exposure dose calculations and equation definitions for incidental sediment ingestion by children in Chartiers Creek, Canonsburg, Pennsylvania, site

\begin{tabular}{lcc}
$\begin{array}{c}\text { Contaminant of } \\
\text { potential concern }\end{array}$ & $\begin{array}{c}\text { Csd } \\
(\mathbf{m g} / \mathbf{k g})\end{array}$ & $\begin{array}{c}\text { Sediment ingestion exposure } \\
\text { doses }\end{array}$ \\
Manganese & $2820^{\mathrm{a}}$ & $2 \mathrm{~m}-03$ \\
Molybdenum & $96^{\mathrm{b}}$ & $6 \mathrm{~kg}-05$ \\
Uranium & $2.6^{\mathrm{a}}$ & $2 \mathrm{E}-06$ \\
\hline
\end{tabular}

\section{Equation definitions for exposure dose calculations}

Incidental ingestion of sediment
Chronic daily intake $(\mathrm{mg} / \mathrm{kg}-$ day $)=\frac{\text { Csd } \times \text { Cf } \times \text { IRsd } \times \text { EF } \times \text { ED }}{B W \times A T}$

Where:

Csd = Contaminant concentration in sediment (maximum concentration) $(\mathrm{mg} / \mathrm{kg})$.

Cf = Conversion factor $\left(10^{-6}\right.$ milligrams per kilogram $\left.[\mathrm{mg} / \mathrm{kg}]\right)$.

IRsd = Incidental ingestion rate for sediment $(100 \mathrm{mg}$ per day for 6 - to 12-year-old children) (EPA, 1989a).

$\mathrm{EF} \quad=$ Exposure frequency (90 days per year; 3 months per year for 7 days per week).

$\mathrm{ED}=$ Exposure duration (7 years for 6- to 12-year-old children).

$\mathrm{BW}=$ Body weight ( $38.3 \mathrm{~kg}$; based on 90th percentile body weight for 6- to 12-yearold male children) (EPA, 1989b).

AT = Averaging time (365 days $\times$ ED) (EPA, 1989a).

${ }^{\mathrm{a} B a s e d}$ on maximum detected value in sediment (sampling location 604, Figure 3.5).

${ }^{b}$ Based on maximum detected value in sediment water (sampling location 605, Figure 3.5).

$\mathrm{mg} / \mathrm{kg}$-day $=$ milligrams per kilogram per day. 
Table 4.4 Exposure dose calculations and equation definitions for ingestion of fish from Chartiers Creek by adults, Canonsburg, Pennsylvania, site

\begin{tabular}{lcccc}
\hline $\begin{array}{c}\text { Contaminant of } \\
\text { potential concern }\end{array}$ & $\begin{array}{c}\text { Csw } \\
(\mathbf{m g} / \mathrm{L})\end{array}$ & BCF & $\begin{array}{c}\text { Fish ingestion } \\
\text { exposure doses } \\
\text { (mg/kg-day) }\end{array}$ & $\begin{array}{c}\text { Ratio of fish } \\
\text { ingestion:surface } \\
\text { water ingestion }\end{array}$ \\
\hline Manganese & 0.17 & $150^{\mathrm{b}}$ & $9 \mathrm{E}-03$ & 180 \\
Molybdenum & 0.17 & NTA & NC & NC \\
Uranium & ND & NA & NA & NA \\
\hline
\end{tabular}

Equation definitions for exposure dose calculations

Ingestion of fish

Chronic daily intake $\left(\mathrm{mg} / \mathrm{kg}\right.$-day) $=\frac{\mathrm{Cf} \times \mathrm{IR} \times \mathrm{EF} \times \mathrm{ED} \times \mathrm{FI}}{\mathrm{BW} \times \mathrm{AT}}$

$\mathrm{Cf}=\mathrm{Csw} \times \mathrm{BCF}$

Where:

Cf = Contaminant concentration in fish (milligrams per kilogram [mg/kg]).

IR = Ingestion rate of fish (0.054 $\mathrm{kg}$ per day for an adult) (EPA, 1991).

$\mathrm{EF}=$ Exposure frequency (350 days per year) (EPA, 1991).

$E D=$ Exposure duration ( 30 years for an adult) (EPA, 1989a).

$\mathrm{FI}=$ Fraction ingested from contaminated source ( 0.5 unitless).

BW = Body weight (70 kg for an adult) (EPA, 1989a).

$A T=$ Averaging time (365 days $\times$ ED for noncarcinogens) (EPA, 1989a).

$\mathrm{Csw}=$ Contaminant concentration in surface water (maximum concentration)(mg/L).

$\mathrm{BCF}=$ Bioconcentration factor (chemical-specific; unitless).

${ }^{a}$ Ratio of the fish ingestion dose to the surface water ingestion dose.

b $B C F$ based on study on minnows (AQUIRE, 1992); converted from dry to wet weight assuming $75 \%$ moisture in fish tissue (EPA, 1993).

ND - not detected in surface water.

NTA - not available.

NA - not applicable, since uranium was not detected in surface water.

NC - could not be calculated.

$\mathrm{mg} / \mathrm{kg}$-day $=$ milligrams per kilogram per day. 
around the more recently observed concentrations. Because relatively little data are available on which to determine shape, the normal distribution was assumed unless strongly contradicted by the data. The data sets for manganese, molybdenum, and uranium were all determined to reasonably fit a normal distribution. The lower tail of the distribution was truncated at $0 \mathrm{mg} / \mathrm{L}$, and the upper tail of the distributions was truncated at the 99th percentile. For every contaminant, the upper truncated concentration was higher than the maximum observed concentration in the water quality data. The software package @ RISK (Palisade Corp., 1992) was used to generate the probability curves for the contaminants of potential concern. Figures 4.2 through 4.4 show the results.

\subsection{ESTIMATION OF GROUND WATER INGESTION INTAKE}

Within the population of future residents, individuals are expected to vary in their water consumption habits, stable body weight, and length of residence time in the potential contamination zone. Consequently, health risks associated with ground water consumption will vary among members of this population. To adequately describe the range of potential risks to a potential future population ingesting water from the unconsolidated layer, naturally occurring variability in daily intake and body weight were incorporated in this assessment through probability distributions; these distributions were generated from the U.S. public health and census documents. All distributions were truncated at the upper and lower 0.01 percentile. Within the hypothetical population, values disallowed through this truncation may occur with a probability of less than 1 in 10,000 .

All of the chemical contaminants of potential concern were evaluated for potential noncarcinogenic effects. In order to evaluate noncarcinogenic effects, exposure to contaminants was estimated as a chronic average daily intake on a body weight basis. Chronic daily intakes were calculated using the following equation:

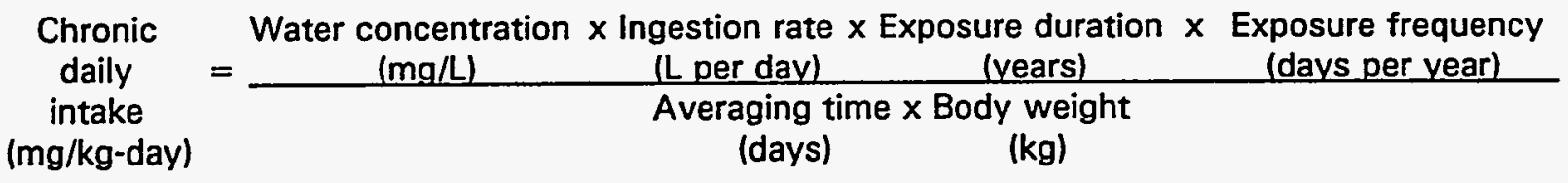

where $\mathrm{mg} / \mathrm{kg}$-day = milligrams per kilogram per day.

Potential carcinogenicity of radionuclides is thought to increase with total intake over time and, thus, is not averaged over a lifetime as for chemical carcinogens. Also, body weight is relatively insignificant in determining risk from exposure. Intake of a radionuclide is therefore quantified as total exposure to radioactivity throughout the residency period of an individual:
Lifetime intake
Concentration $x$ Ingestion rate $x$ Exposure duration $x$ (pCi per lifetime) $=(\mathrm{pCi} / \mathrm{L})$
(L per day)
(years)
Exposure frequency (days per year) 


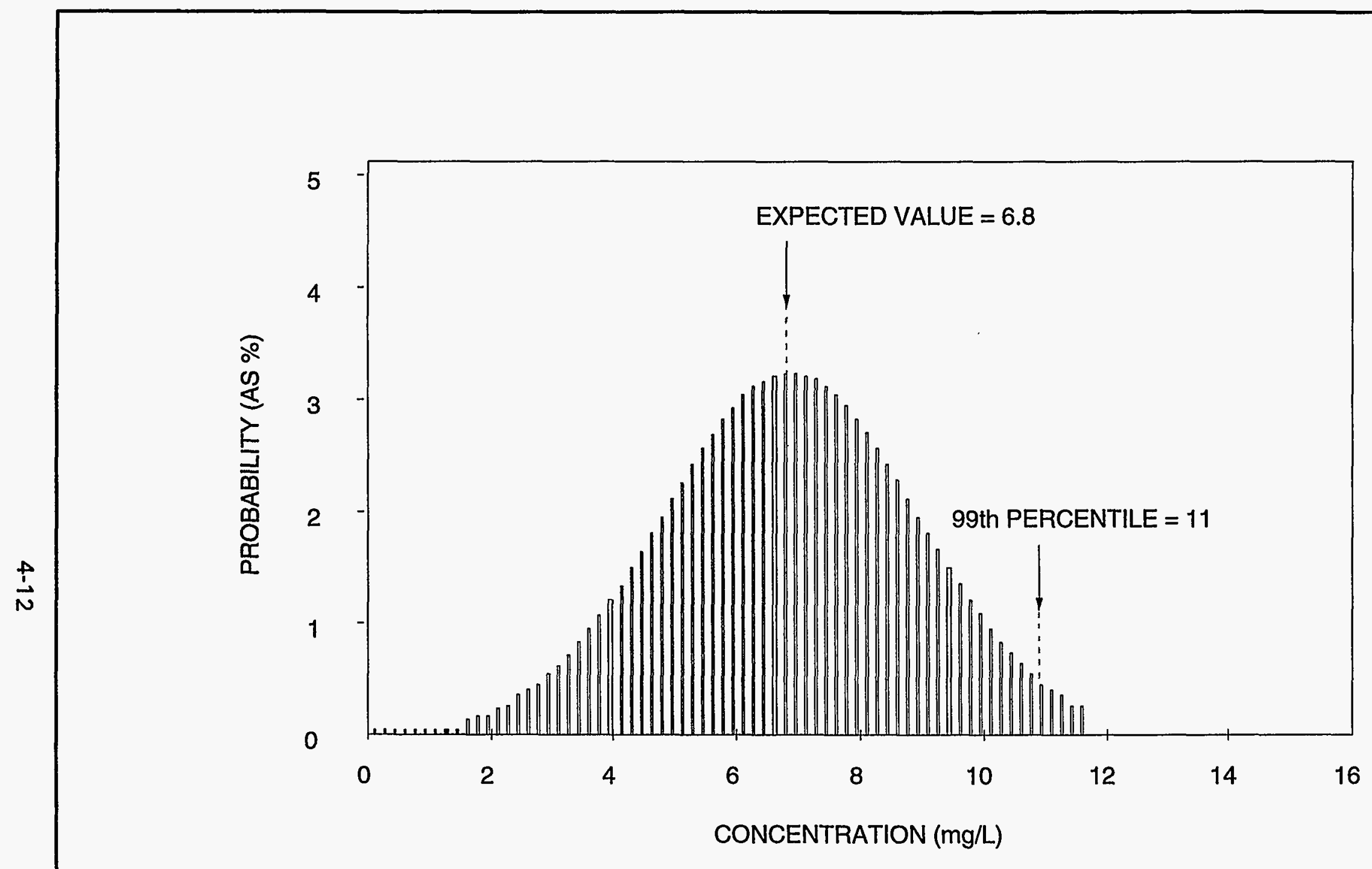

FIGURE 4.2

PROBABILITY DISTRIBUTION OF MANGANESE CONCENTRATIONS FOR THE UNCONSOLIDATED MATERIAL CANONSBURG, PENNSYLVANIA, SITE 


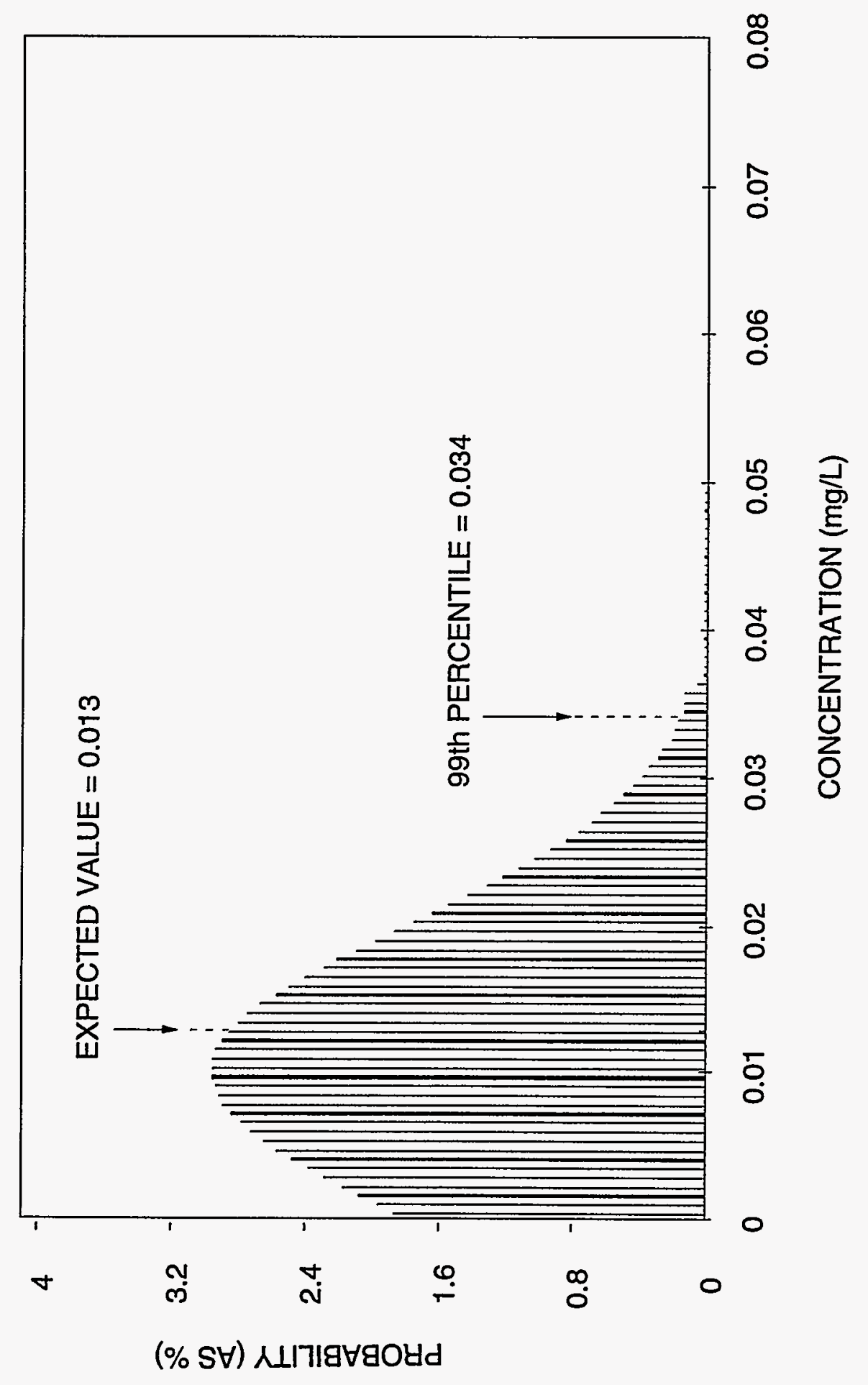

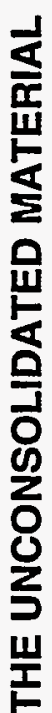

号屴

o 5

을

논

峁

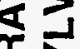

足

㟧岂忌

는 엉 동

事

정

엉

4

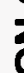

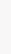

흐

政

5

D

E

产

$\mathbf{x}$

옹 


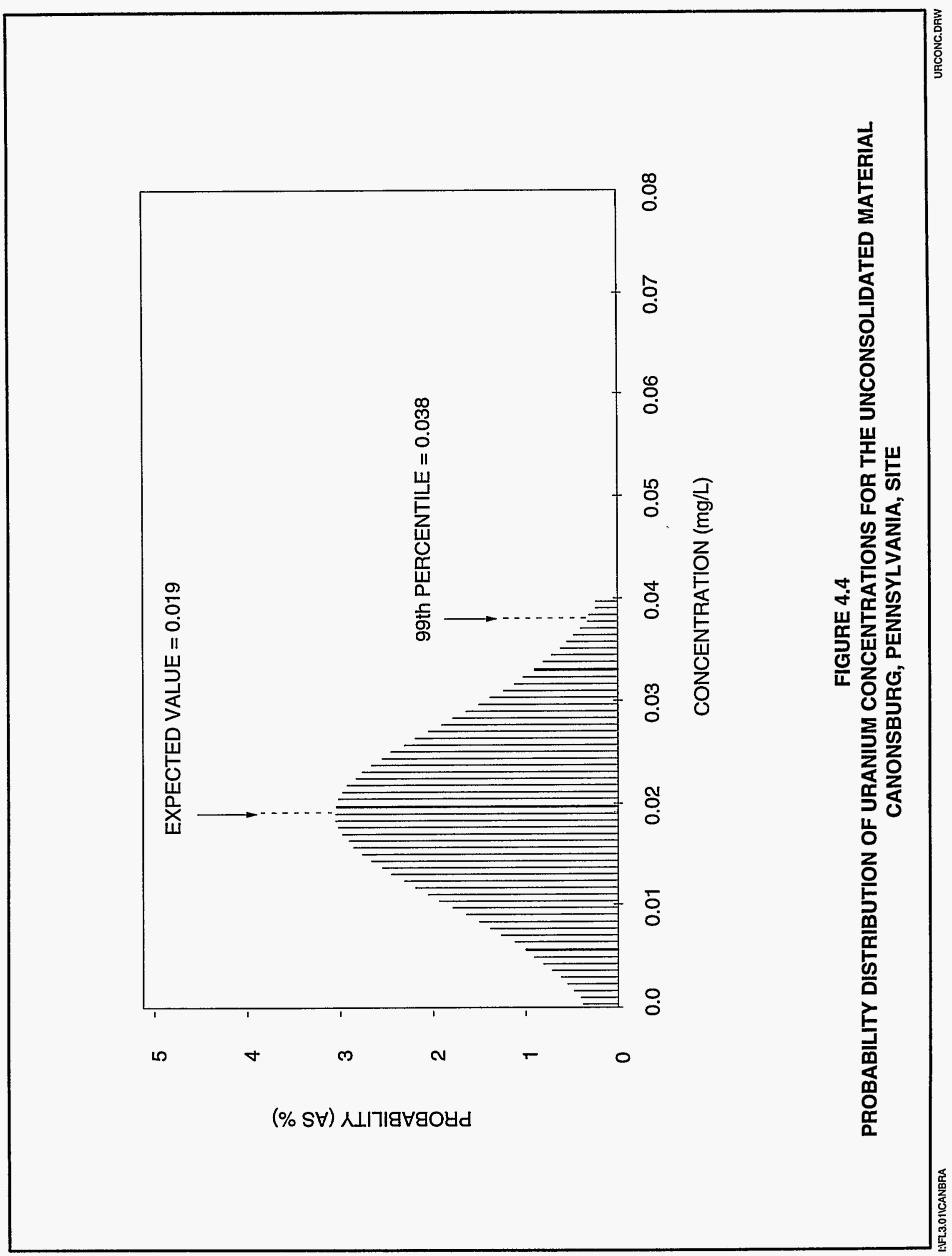




\section{Average daily water intake ( $L$ per day)}

Lognormal probability distributions were used to describe variations in average daily tap water intake among members of the population (Roseberry and Burmaster, 1992). These distributions were developed from data collected during the 1977-78 National Food Consumption Survey conducted by the U.S. Department of Agriculture. During the survey, total tap water consumption during a 3-day period was recorded for 26,081 survey participants nationwide (Figure 4.5).

The two graphs in Figure 4.5 show on their horizontal axes reasonable water ingestion rates associated with two age groups. The height of the curve at each point along this axis communicated how common the different levels of water ingestion are among members of that age group. For this reason, the vertical scale is labeled as probability. However, theoretically the probability is represented by the area under the curve, not the height of the curve itself. Therefore, the probability that a randomly selected person consumes a given amount of water, within a specified interval, is estimated by multiplying the height of the curve overlying that interval by the width of the interval. This formula is true for all the probability distribution graphs presented in this document.

\section{Exposure frequency (days per year)}

An exposure frequency of 350 days per year was assumed for adults and children (EPA, 1991).

\section{Exposure duration (years)}

Children are the receptors of concern evaluated for the noncarcinogenic effects of the contaminants of concern. A 10-year exposure duration was assumed for 1- to 10-year-old children.

For carcinogens such as uranium and other radionuclides, risk is cumulative throughout the lifetime. For the evaluation of carcinogens, an exposure duration of 30 years was assumed, based on a national upper-bound time (90th percentile) spent at one residence (EPA, 1989a).

\section{Body weight (kg)}

Between 1976 and 1980, the National Health and Nutrition Survey collected extensive national data on the weights of males and females by age (EPA, $1989 \mathrm{~b})$. These data were used to develop lognormal probability distributions for body weight by age and by gender. The distributions for males and females were then combined using census data on the national ratio of males and females within each age group (Figure 4.6). 

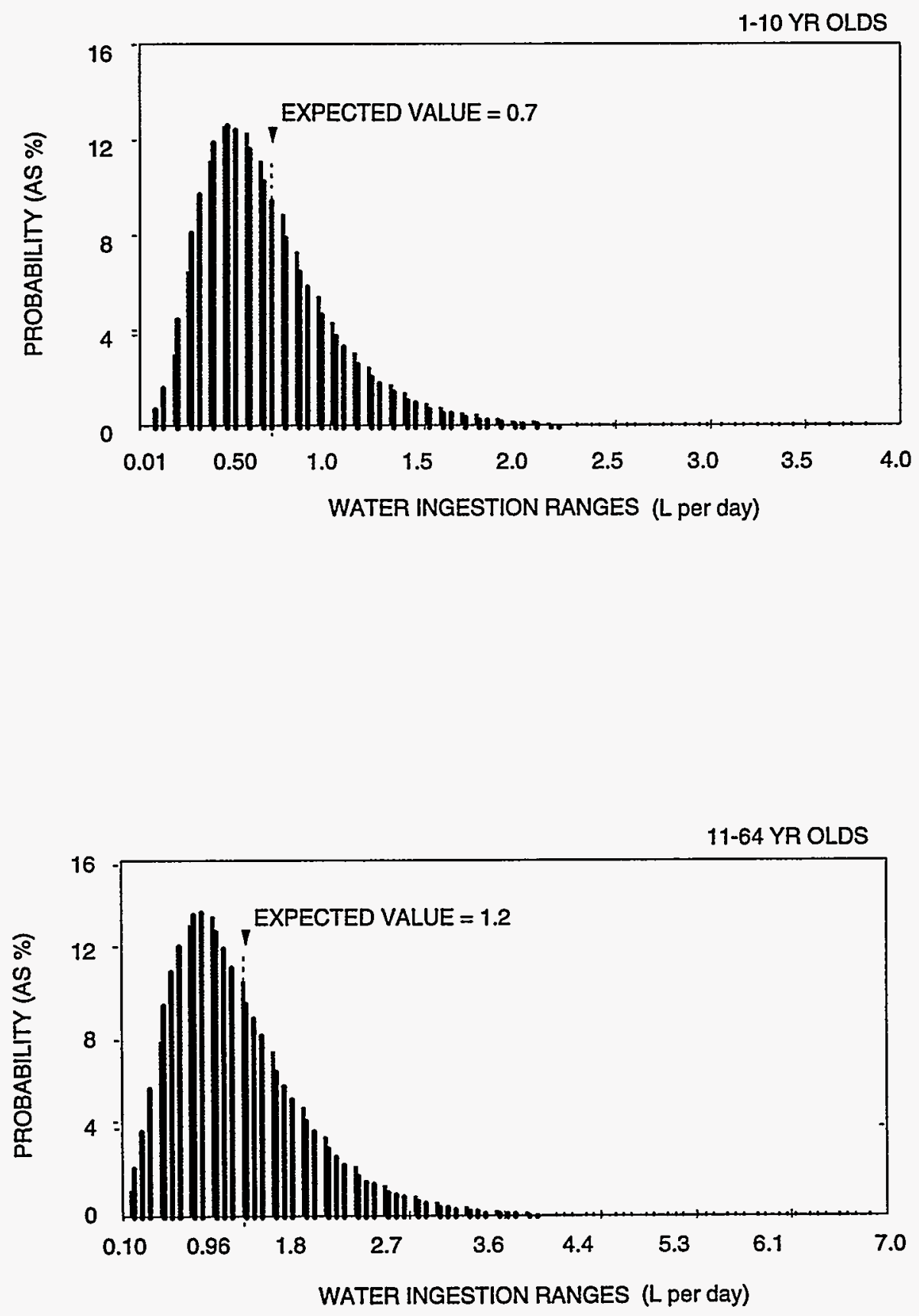

FIGURE 4.5

PROBABILITY DISTRIBUTIONS FOR TAP WATER INGESTION RATES 


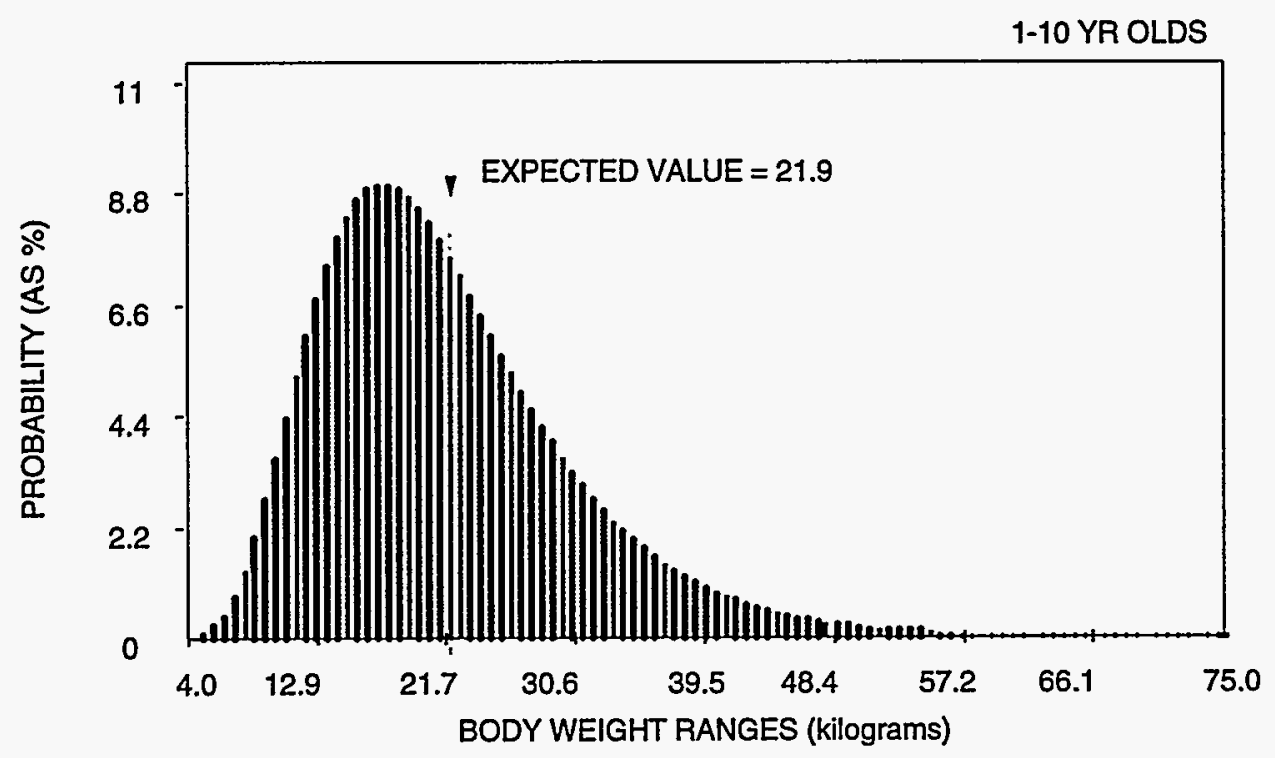

11-64 YR OLDS

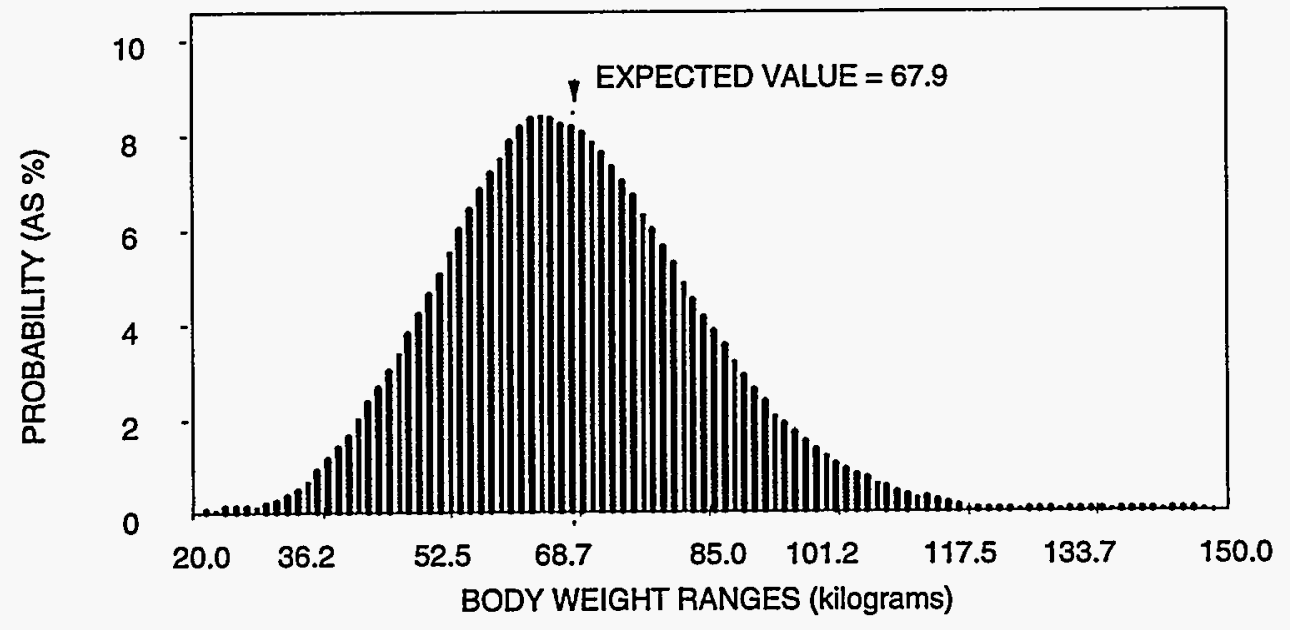

FIGURE 4.6

PROBABILITY DISTRIBUTIONS FOR BODY WEIGHT 


\section{Averaging time (days)}

When evaluating potential noncarcinogenic effects from longer-term exposures, intakes are averaged over the period of exposure. Thus, in evaluating the noncarcinogenic effects of the contaminants of concern, an averaging time of 3650 days (i.e., 10 years $\times 365$ days) was used for children.

\section{Intake distributions}

Using the exposure concentration distributions discussed in Section 4.3 and the intake parameter distributions described in this section, total intake distributions were simulated for the receptor populations. The 1- to 10-year-old age group consistently showed the highest intake-to-body weight ratios and is therefore the most conservative age group to evaluate for noncarcinogenic risk. Children are likely more susceptible to metals toxicity than adults because of higher gastrointestinal absorption efficiency and other toxicokinetic factors (Casarett and Doull, 1991). Figures 4.7 through 4.9 present simulated intake distributions for 1- to 10-year-old children for manganese, molybdenum, and uranium, respectively. Figure 4.10 shows the total lifetime intake estimate used to calculate carcinogenic risk for uranium.

\subsection{EXPOSURE ASSESSMENT UNCERTAINTIES}

A number of potential sources of uncertainty may arise in all phases of the exposure assessment, including the following more notable sources:

- Uncertainties resulting from a lack of thorough environmental characterization of the site, which could lead to an underestimation or overestimation in the exposure analysis. In addition, background ground water quality is represented by data from one monitor well. Therefore, natural variability in the background ground water quality could not be captured.

- Uncertainties arising from the assumption that the ground water contaminant sources at the site have reached a steady state and that contaminant concentrations at the exposure point will remain constant for chronic periods of exposure (generally longer than 7 years). Because the major source of contamination at the Canonsburg site has been encapsulated on the site, the assumption of a constant source may lead to an overestimation of risk.

- Uncertainties associated with the use of filtered ground water samples. The results of exposure assessment presented in this document are based on filtered (0.45-micron) ground water samples. Therefore, the potential loss of certain ground water constituents as a consequence of filtration represents an additional source of uncertainty. 


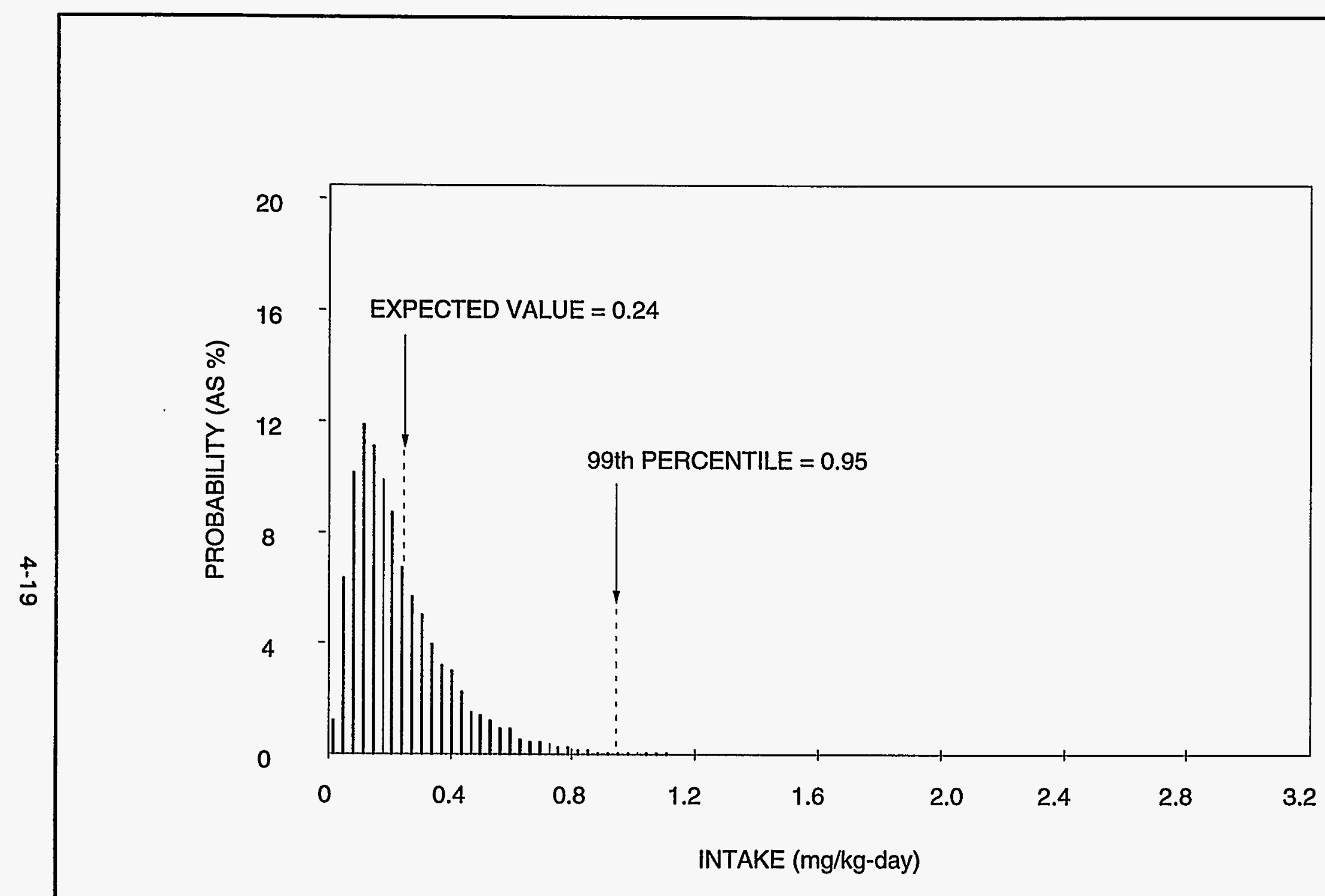

FIGURE 4.7

PROBABILITY DISTRIBUTION OF MANGANESE EXPOSURE TO CHILDREN BASED ON GROUND WATER INGESTION CANONSBURG, PENNSYLVANIA, SITE 


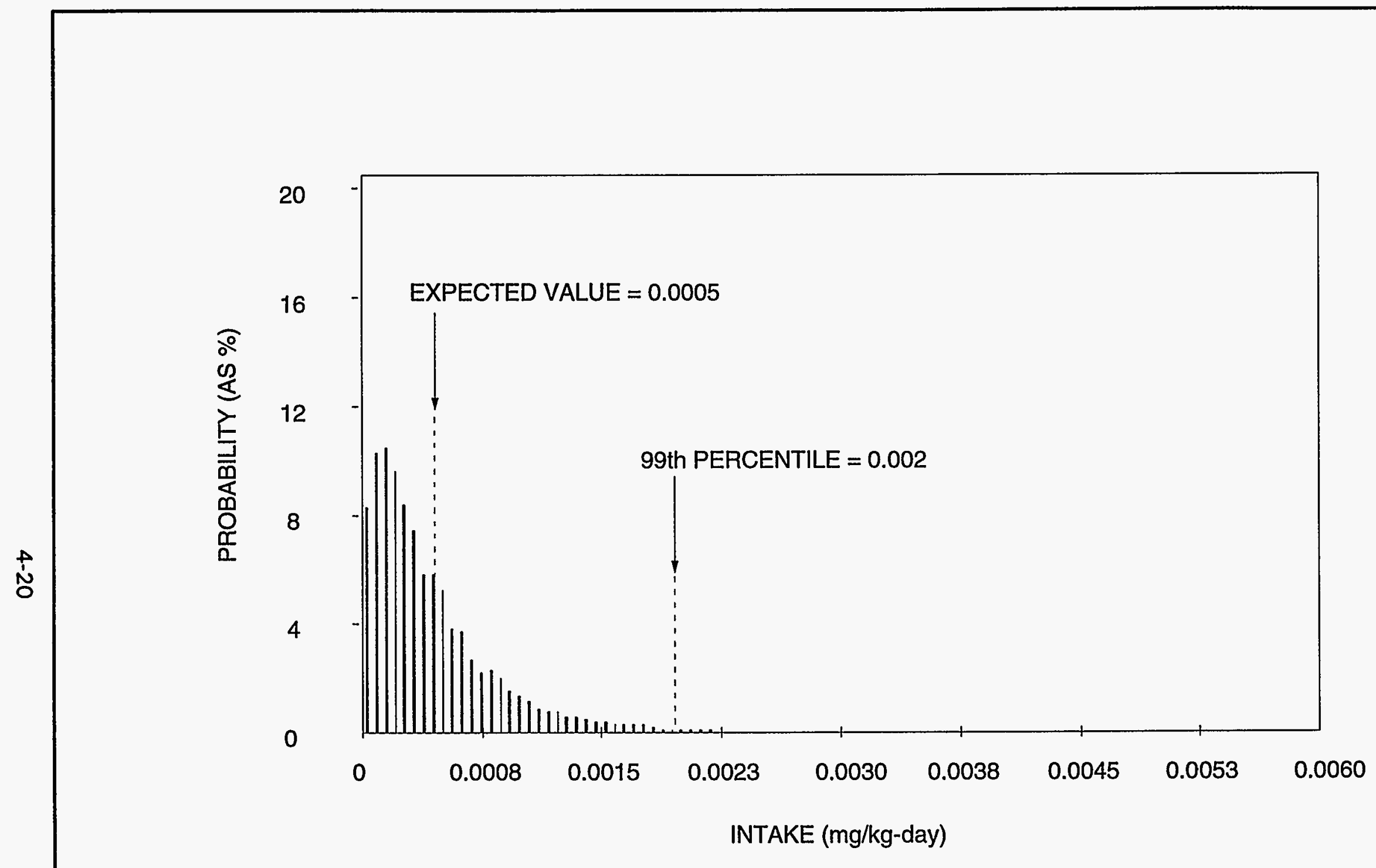

FIGURE 4.8

PROBABILITY DISTRIBUTION OF MOLYBDENUM EXPOSURE TO CHILDREN BASED ON GROUND WATER INGESTION CANONSBURG, PENNSYLVANIA, SITE 


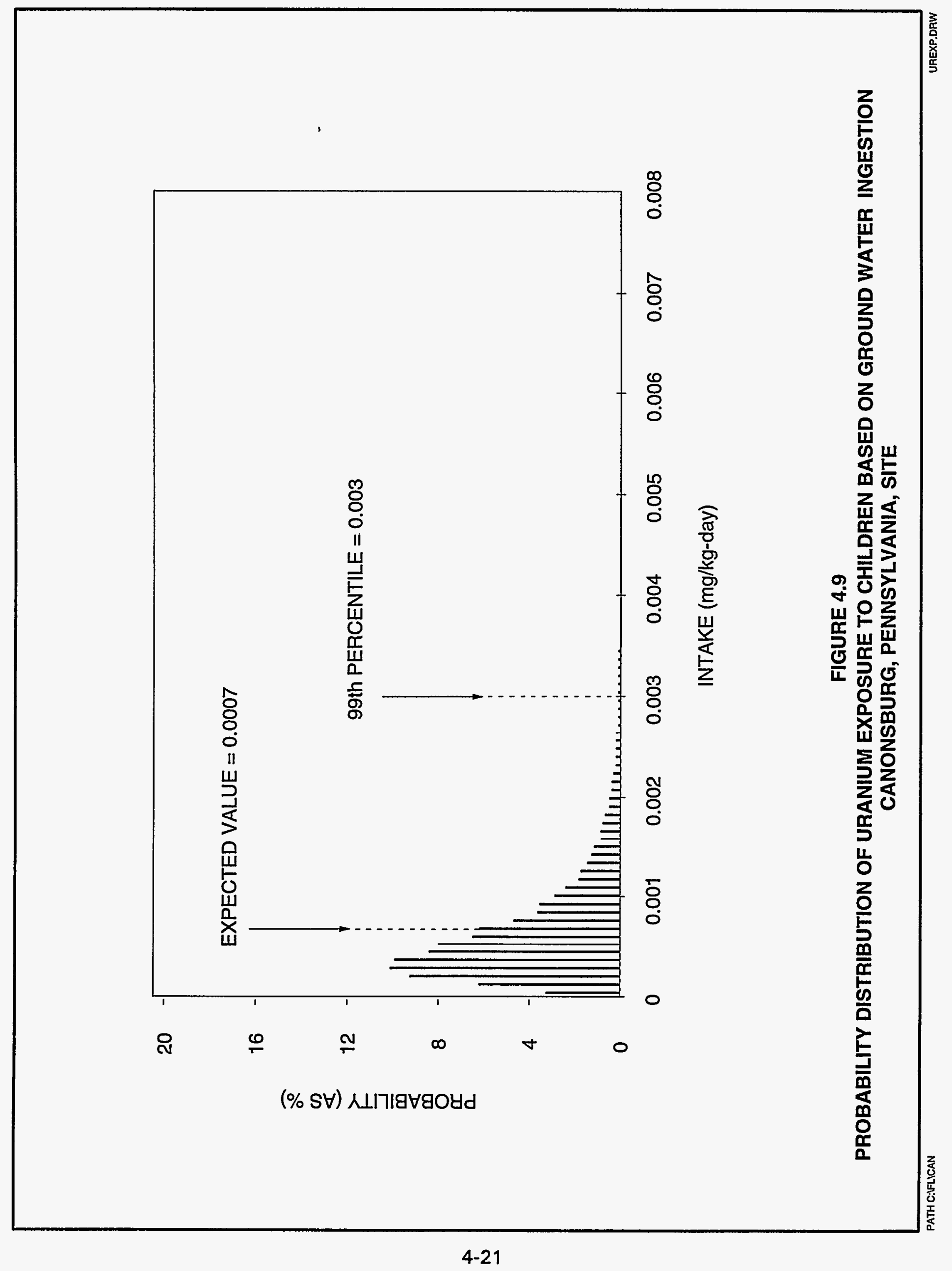




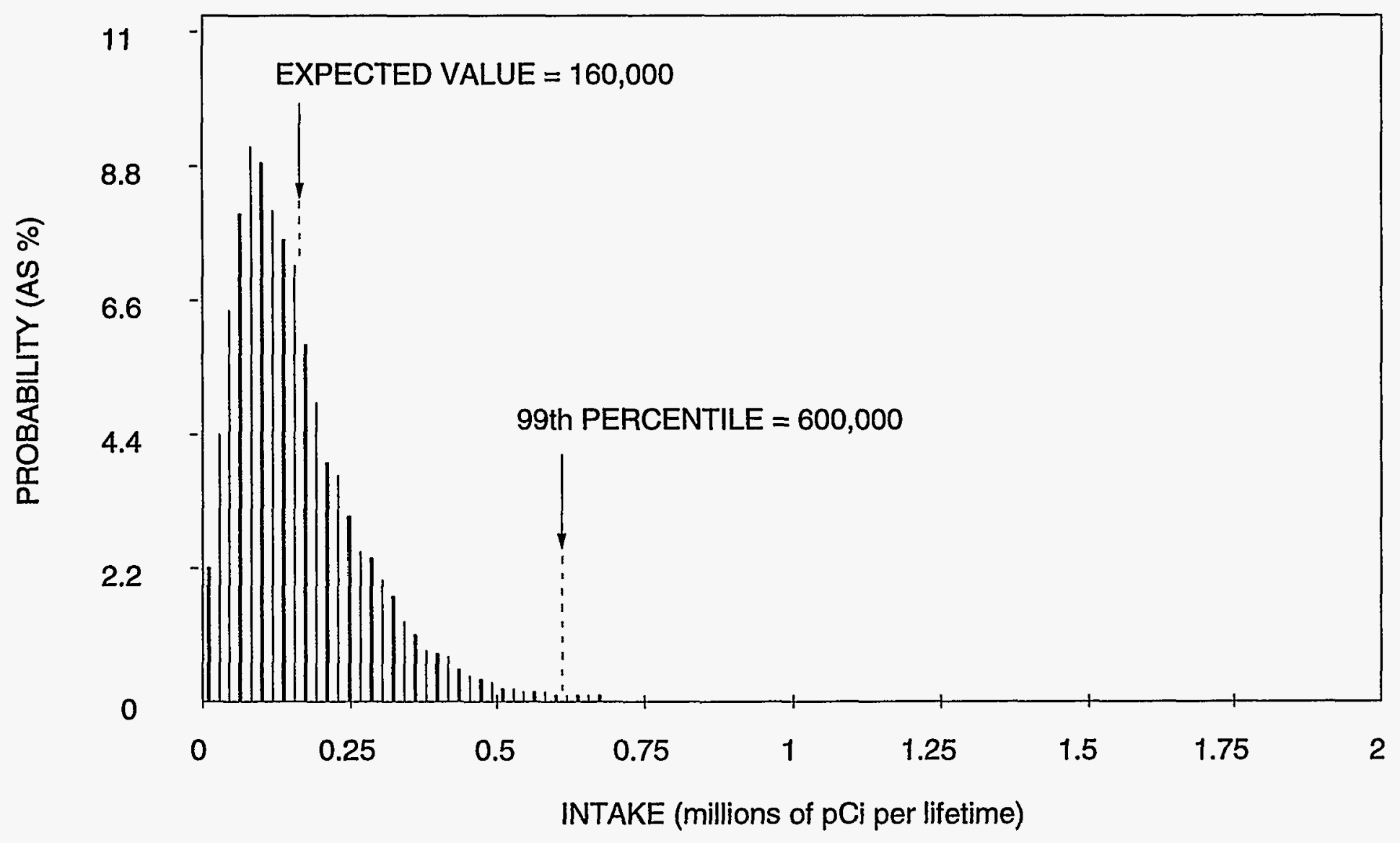

FIGURE 4.10

PROBABILITY DISTRIBUTION OF URANIUM EXPOSURE OVER A LIFETIME BASED ON GROUND WATER INGESTION CANONSBURG, PENNSYLVANIA, SITE 
- Uncertainties associated with the incremental contribution from ingesting ground water-irrigated produce. The drinking water pathway is considered the major determinant of exposure in this risk assessment. The incremental or independent contribution from the ground water-irrigated produce ingestion pathway could not be estimated here.

- Uncertainties associated with the relationship of an applied dose (used in this risk assessment) and an absorbed or effective dose for dermal absorption.

- Uncertainties associated with the exclusion of organic contaminants from consideration as a source of potential exposure. This document evaluates exposures only to inorganic contaminants of ground water at the UMTRA Project site in Canonsburg. Potential organic contaminants related to uranium processing have not been considered.

Despite these uncertainties, using probability distributions that incorporate all definable sources of variability should provide a representative picture of the potential range of exposures from the drinking water ingestion pathway. 


\subsection{TOXICITY ASSESSMENT}

Several contaminants that could adversely affect human health and the environment have been detected in ground water at the Canonsburg site. This section summarizes the toxicological effects of the chemical contaminants and the carcinogenic potentials of the radionuclides.

The following source materials were used in developing these toxicological profiles: EPA's Integrated Risk Information System (IRIS) (EPA, 1994a); the Agency for Toxic Substances and Disease Registry Toxicological Profiles published by the Department of Health and Human Services; and the Handbook on the Toxicology of Metals (Friberg et al., 1986). Peer-reviewed scientific literature was used when these review documents were not available firsthand. By basing toxicity information on the standardized review documents cited above, the evaluation of risks at UMTRA Project sites should be consistent with evaluations at other sites.

The toxicity profiles presented here focus on drinking water source material in humans, whenever available, and include animal information only when human data are not available. Widely spaced dotted lines represent animal information on the toxicity range graphs. Where uncertainty exists regarding the beginning or end of a range of exposures that produces specific toxic effects, closely spaced dots are used at the appropriate end of the line that denotes range.

\section{$5.1 \quad$ CONTAMINANT TOXICITY SUMMARIES}

The following summaries address the basic toxicokinetics and toxicity of the contaminants of potential concern at the Canonsburg site based on the contaminant screening discussed in Section 3.3. The contaminants of potential concern are manganese, molybdenum, and uranium and its decay products (radium-226, lead-210, polonium-210, and thorium-230). Although these contaminants have a wide range of toxic effects depending on the exposure levels, the following discussions will focus on toxic effects observed in the exposure range most relevant to contamination at this site.

\subsubsection{Manganese}

\section{Absorption}

Following ingestion, manganese absorption is homeostatically controlled. The rate of absorption depends on both the amount ingested and tissue levels of manganese. In adult humans, approximately 3 to 4 percent of dietary manganese is absorbed (Saric, 1986). Manganese can be absorbed following exposure by inhalation, ingestion, and dermal contact. In humans, available data indicate that only 3 percent of an ingested dose of manganese chloride is absorbed (Mena et al., 1969). Manganese in water appears to be more efficiently absorbed than manganese in food (EPA, 1994a). Iron and other metals influence the rate of absorption. In states of iron deficiency, manganese 
is actively absorbed from the intestine. Individuals with anemia can absorb more than twice the percentage of an ingested dose. However, in states of excess iron, absorption of manganese is by diffusion only (Saric, 1986). High levels of dietary calcium and phosphorus have been shown to increase the requirements for manganese in several species (Lönnerdal et al., 1987). Manganese in water appears to be more efficiently absorbed than manganese in foodstuff (EPA, 1994a).

\section{Tissue accumulation and clearance}

Absorbed manganese is widely distributed throughout the body, with the highest concentrations found in the liver and kidney. The biological half-life in humans is 2 to 5 weeks, depending on body stores. Manganese readily crosses the blood-brain barrier and is cleared from the brain more slowly than from other tissues (Goyer, 1991). Although normal concentrations in the brain are low, manganese may accumulate under conditions of excessive absorption due to the longer half-life of manganese in the brain (National Research Council, 1973).

Absorbed manganese is rapidly cleared from the blood and concentrates in mitochondria. Initial concentrations are greatest in the liver. Manganese penetrates the placental barrier in all species and is more uniformly distributed throughout the fetus than in adult tissues. It is secreted into milk.

Absorbed manganese is almost totally secreted in bile and reabsorbed from the intestine as necessary to maintain body levels. Excess manganese is eliminated in the feces; urinary excretion is negligible (Goyer, 1991; Saric, 1986).

\section{Environmental sources of manganese}

Food constitutes the major source of manganese intake for humans. The highest manganese concentrations are found in plants, especially wheat and rice. Drinking water generally contains less than $0.1 \mathrm{mg} / \mathrm{L}$. Manganese levels in soil range from 1 to $7000 \mathrm{mg} / \mathrm{kg}$, with an average of 600 to $900 \mathrm{mg} / \mathrm{kg}$. Mining and natural geological background variation can contribute to this variability. Manganese bioaccumulates in marine mollusks up to 12,000 -fold, and there is evidence for toxic effects in plants (phytotoxicity) and plant bioaccumulation. The Illinois Institute for Environmental Quality recommends criteria of 1 to $2 \mathrm{mg} / \mathrm{kg}$ for manganese in soil and $200 \mathrm{mg} / \mathrm{kg}$ in plants (Saric, 1986).

Differences in nutritional habits can explain variations in manganese intake. The intake will be higher in populations where cereals and rice are main food sources than where meat and dairy products make up a larger part of the diet. The average daily intake is estimated to be between 2.0 and $8.8 \mathrm{mg}$ per day 10.03 and $0.13 \mathrm{mg} / \mathrm{kg}$-day) (EPA, 1994a), but intakes as high as $12.4 \mathrm{mg}$ (about $0.2 \mathrm{mg} / \mathrm{kg}$-day) have been reported in countries with high cereal intake (Saric, 1986). 
Drinking water generally results in an intake of less than $0.2 \mathrm{mg}$ $(0.003 \mathrm{mg} / \mathrm{kg}$-day), although some mineral waters can increase this amount more than threefold (Saric, 1986). One study from Greece reported drinking water concentrations of manganese in excess of $2 \mathrm{mg} / \mathrm{L}$, which would result in daily intakes from 0.06 to $0.07 \mathrm{mg} / \mathrm{kg}$-day (EPA, 1994a).

\section{Manganese toxicity}

Manganese is an essential nutrient: Estimated safe and adequate daily dietary intakes for adults range from 0.03 to $0.07 \mathrm{mg} / \mathrm{kg}$-day (Saric, 1986). The EPA no-observed-adverse-effect level (NOAEL) for drinking water is identified at $0.005 \mathrm{mg} / \mathrm{kg}$-day while the lowest-observed-adverse-effect level (LOAEL) is $0.06 \mathrm{mg} / \mathrm{kg}$-day. The EPA oral reference dose (RfD) for drinking water of 0.005 $\mathrm{mg} / \mathrm{kg}$-day is based on human data (Kondakis et al., 1989). The study group was a population of older adults exposed to manganese in drinking water over a lifetime; because this population was considered sensitive, an uncertainty factor of 1 was applied (EPA, 1994a). The oral RfD for food ingestion is $0.14 \mathrm{mg} / \mathrm{kg}$ day, based on a level at which no adverse effects were observed. Manganese in drinking water appears to be potentially more bioavailable, i.e., more readily absorbed, than manganese in dietary food sources. This would result in toxic effects with lower ingested doses of manganese in drinking water than in food (EPA, 1994a). However, insufficient data exist to quantify these differences.

Inhalation of manganese in industrial settings has provided the largest source of data on chronic manganese toxicity. These data indicate that excess manganese can result in a central nervous system disorder manifested by symptoms of irritability, difficulty in walking, speech disturbances, and compulsive behavior that may include running, fighting, and singing. With continued exposure, this condition can progress to a mask-like face, retropulsion or propulsion, and a Parkinson-like syndrome. These effects are largely irreversible, although some recovery may occur when exposure ceases (DHHS, 1992). Metal chelating agents are ineffective in treatment, but L-dopa has been effective (Goyer, 1991), suggesting that manganese produces a functional deficit in the central nervous system.

Limited information is available on the effects of manganese ingestion. Because effects from drinking water seem to differ from those from food sources, only water consumption studies will be considered here. A Japanese study of 25 people drinking well water with manganese concentrations of $14 \mathrm{mg} / \mathrm{L}$ $10.4 \mathrm{mg} / \mathrm{kg}$-day estimated intake) reported symptoms of intoxication, including a mask-like face, muscle rigidity and tremors, and mental disturbances (DHHS, 1992). Two cases (8 percent) of death were reported among intoxicated people. A Greek study of more than 4000 adults at least 50 years old drinking water with manganese concentrations varying from 0.081 to $2.3 \mathrm{mg} / \mathrm{L}$ (estimated intake at $2 \mathrm{~L}$ per day for a $70-\mathrm{kg}$ individual range from 0.002 to 0.07 $\mathrm{mg} / \mathrm{kg}$-day) showed varying degrees of neurological effects in those drinking from 0.007 to $0.07 \mathrm{mg} / \mathrm{kg}$-day, but no effects in those drinking less than 0.005 $\mathrm{mg} / \mathrm{kg}$-day (Kondakis et al., 1989). However, there are many limitations to 
these studies which make data interpretation difficult. Among the limitations, there is uncertainty regarding the exposure level or whether the effects seen were solely attributable to manganese. Despite the limitations of these studies, the similarity of the effects seen in the cases of oral exposure compared with those associated with inhalation exposure suggests that excess manganese intake by humans might lead to neurological injury (DHHS, 1992).

The chemical form of manganese has complex effects on its toxicity. Although more soluble forms are more readily absorbed from the gastrointestinal tract, they also appear to be more rapidly cleared. Exposure to insoluble forms results in lower manganese absorption, but higher chronic tissue levels and therefore greater toxicity (EPA, 1994a). Limited information is available on the effects of various forms of manganese.

Few data are available on manganese toxicity in infants, but infants probably will be more susceptible to manganese toxicity due to greater absorption and greater penetration into the central nervous system (EPA, 1994a; Saric, 1986).

Figure 5.1 summarizes manganese toxicity.

\subsubsection{Molybdenum}

\section{Absorption}

Absorption of molybdenum in the gastrointestinal tract depends on the species of the metal. Inorganic hexavalent forms such as molybdenum trioxide, sodium molybdate, and ammonium molybdate are readily absorbed from both food and water, unlike molybdenite. Based on the geochemical models for the Canonsburg, Pennsylvania, site, all the molybdenum exists in the form of easily absorbed molybdate in ground water (Table 3.4). Human absorption rates of 40 to 70 percent have been observed for soluble forms of molybdenum (Robinson et al., 1973; Alexander et al., 1974).

\section{Tissue accumulation and clearance}

In humans, the highest concentrations of molybdenum occur in the liver, kidneys, and adrenals (Casarett and Doull, 1991). With normal dietary intake, molybdenum levels in the body slowly increase until approximately age 20 , when they begin to decline steadily. The principal route of excretion in humans is in the urine. Human studies indicate the biological half-life in humans is considerably longer than in animals and may be as long as 2 weeks (Rosoff and Spencer, 1964).

\section{Environmental sources of molybdenum}

The occurrence of natural molybdenum is in combination with other metals, including uranium, lead, iron, cobalt, and calcium. Native soil concentrations can vary by as much as 2 orders of magnitude, from 0.1 to $10 \mathrm{mg} / \mathrm{kg}$, leading to 


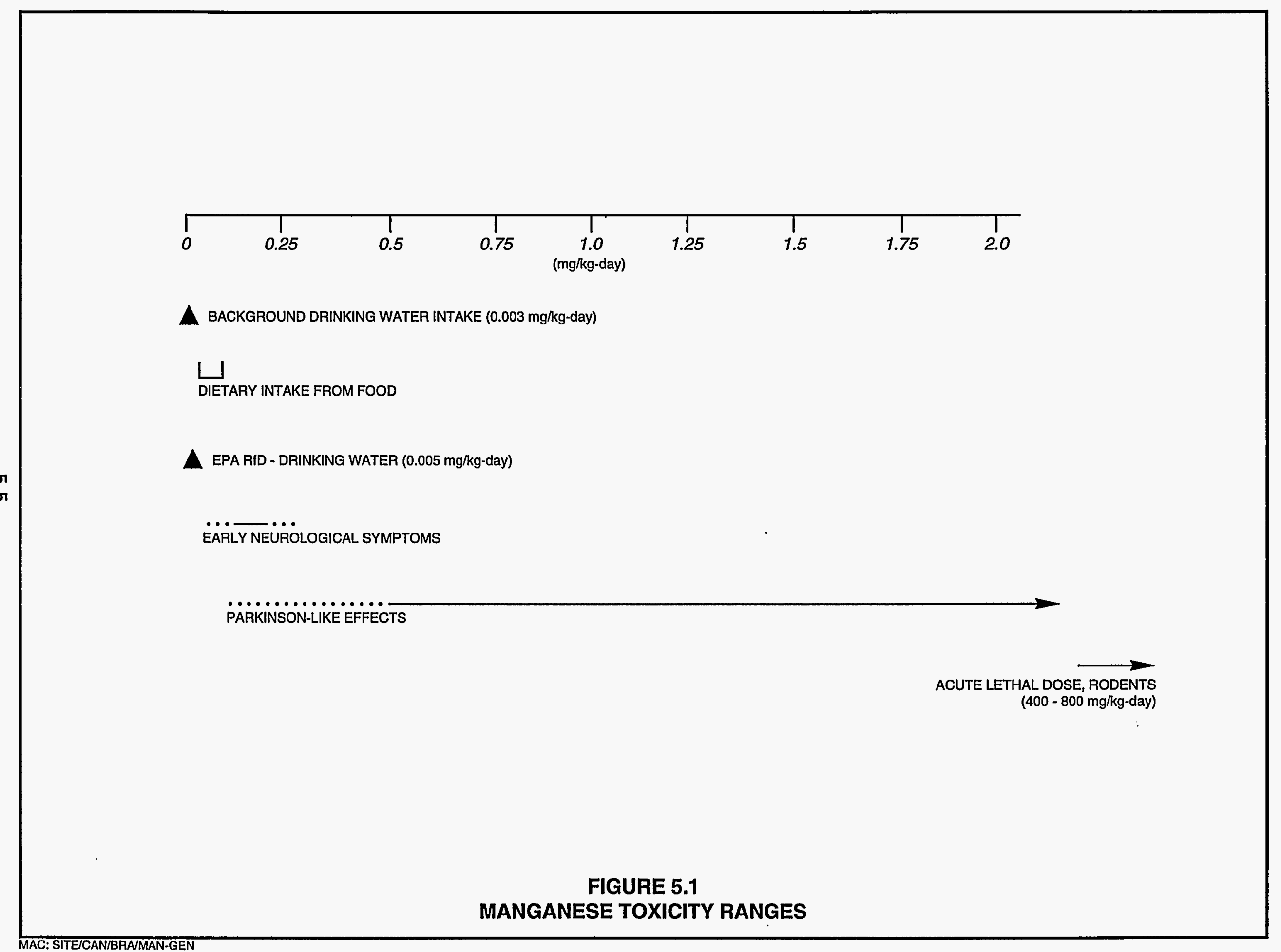


large variations in the molybdenum concentrations in plant materials. Natural concentrations in ground water have been reported from 0.00011 to $0.0062 \mathrm{mg} / \mathrm{L}$. Human dietary intake of molybdenum is estimated at 0.05 to $0.24 \mathrm{mg}$ per day $(0.0007$ to $0.003 \mathrm{mg} / \mathrm{kg}$-day). The contribution of drinking water is estimated to range from 0 to 95 percent. The nutritional range of intake for molybdenum is from 0.0015 to $0.0054 \mathrm{mg} / \mathrm{kg}$-day. No symptoms of molybdenum deficiency have been reported in humans. Nonetheless, molybdenum is an essential trace element that functions as a necessary constituent of several enzymes, including xanthine oxidase (which is involved in the metabolism of uric acid) and nitrate reductase (Friberg et al., 1986).

\section{Molybdenum toxicity}

Acute effects of molybdenum have not been reported. No adverse health effects have been reported with chronic molybdenum intakes of less than $0.008 \mathrm{mg} / \mathrm{kg}$-day (EPA, 1994a). The primary toxicity of molybdenum is related to its interactions with copper and sulfur, leading to altered excretion patterns for these elements. Increased levels of molybdenum also increase the levels of xanthine oxidase, which is responsible for the production of uric acid. Uric acid can accumulate in joints and lead to symptoms of gout and other joint disorders.

Intake of 0.008 to $0.022 \mathrm{mg} / \mathrm{kg}$-day of molybdenum can produce mineral imbalance as a result of increased copper excretion (EPA, 1994a). Excretion of copper is reported to double with molybdenum intakes at the upper end of this range. Copper is an essential nutrient important in many metabolic pathways, including the synthesis and function of hemoglobin. A copper deficiency resulting from excess excretion will impair the oxygen-carrying capacity of the blood, and severe copper deficiencies can lead to hypochromic microcytic anemia. In humans, gout-like symptoms and joint deformities have been reported in regions of Russia where elevated soil concentrations of molybdenum and subsequent increased molybdenum concentrations in food would lead to molybdenum intakes in the range of 0.14 to $0.21 \mathrm{mg} / \mathrm{kg}$-day. The EPA oral reference dose of $0.005 \mathrm{mg} / \mathrm{kg}$-day for molybdenum was derived from results of these studies (EPA, 1994a). An uncertainty factor of 30 was used for two reasons: to protect the sensitive human population and to use the LOAEL of $0.14 \mathrm{mg} / \mathrm{kg}$-day rather than the preferred NOAEL which should be identified from a long-term study in human population. Figure $\mathbf{5 . 2}$ summarizes the health effects of molybdenum as a function of dose.

\subsubsection{Uranium}

Naturally occurring uranium, present at UMTRA Project sites, consists of three radioactive isotopes: uranium-234, uranium-235, and uranium-238. More than 99 percent of natural uranium occurs in the form of uranium-238 (Cothern and Lappenbusch, 1983). Uranium-238 undergoes radioactive decay by emitting alpha particles to form uranium-234, thorium-230, radium-226, radon 222, polonium-210, and other radioisotopes. Figure 5.3 summarizes the radioactive 

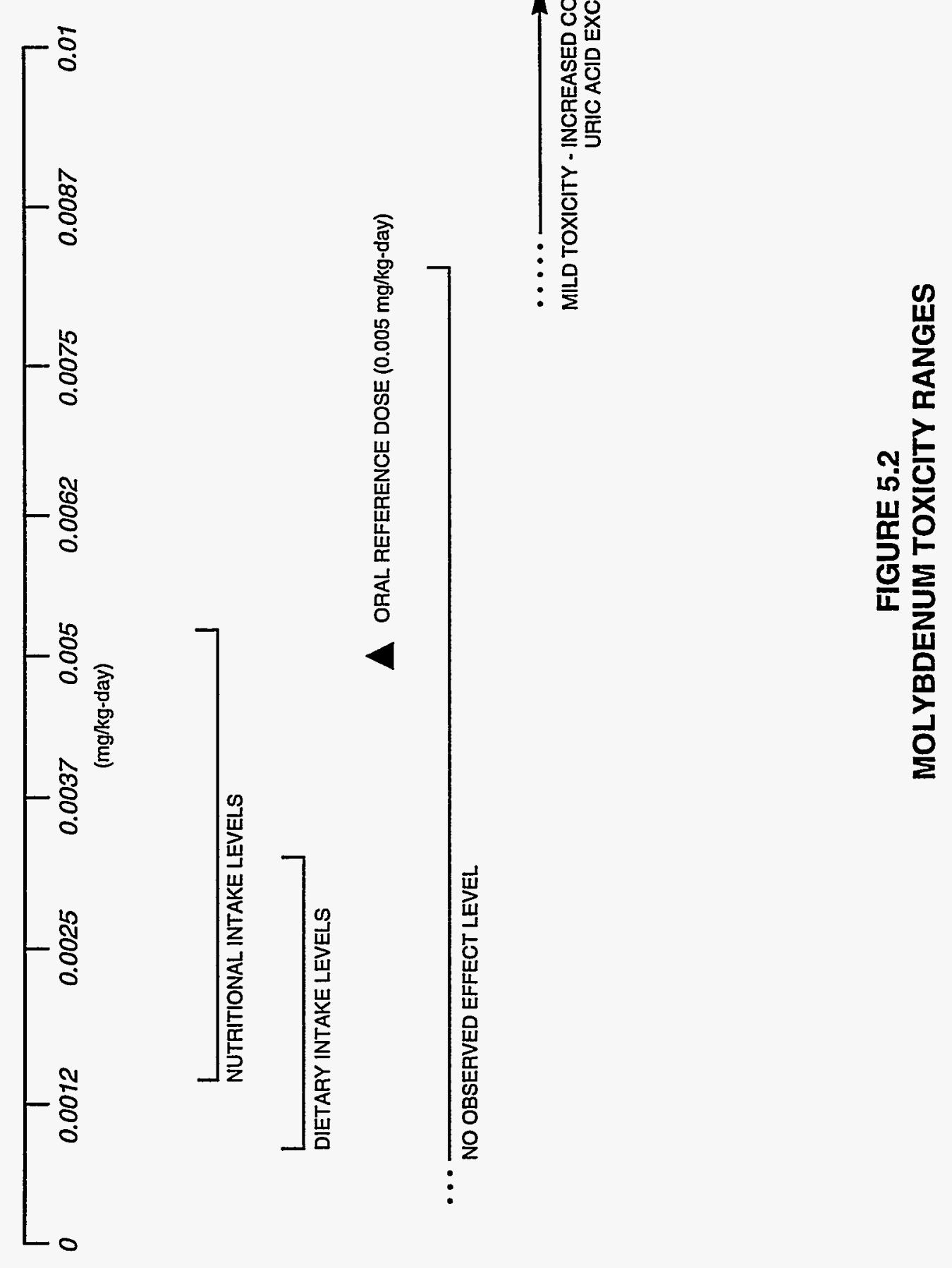

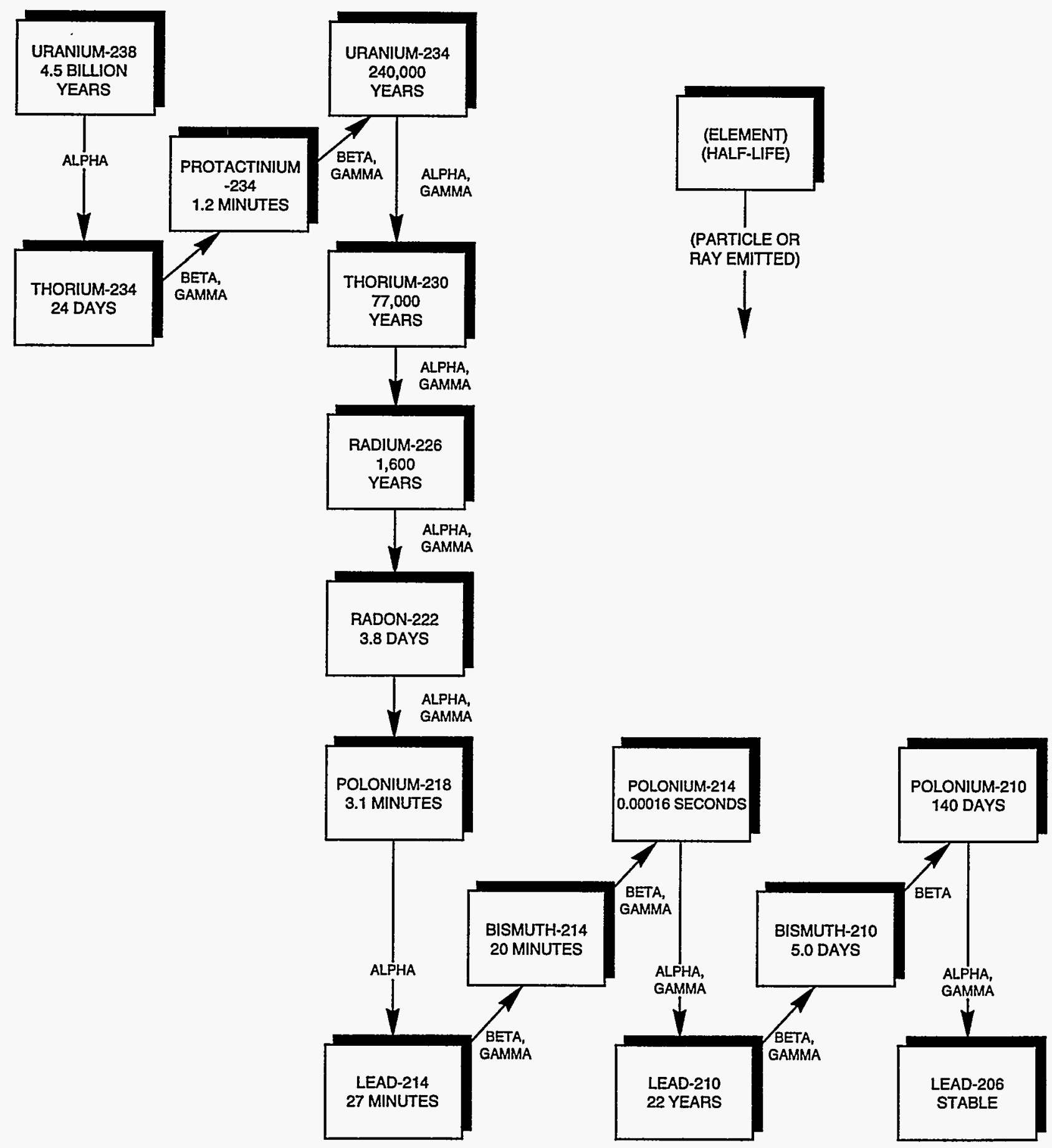

NOTE: ALL THE HALF-LIFE VALUES ARE ROUNDED TO THE TWO SIGNIFICANT DIGITS.

MODIFIED FROM SHLEIEN, 1992.

FIGURE 5.3

HALF-LIVES AND EMISSIONS FROM DECAY CHAIN OF URANIUM-238 
decay chain of uranium-238 and uranium-234. Because all uranium isotopes in nature are radioactive, the hazards of a high uranium intake are from both its chemical toxicity and potential radiological damage. This section focuses on the chemical toxicity of natural uranium. Section $\mathbf{5 . 3}$ discusses the carcinogenic potential associated with exposure to radioactive isotopes of natural uranium.

\section{Absorption}

Absorption of uranium in the gastrointestinal tract depends on the solubility of the uranium compounds. The hexavalent uranium compounds, especially the uranyl salts, are water soluble, while tetravalent compounds generally are not (Weigel, 1983). Even with soluble compounds, only a small fraction is absorbed. Human gastrointestinal absorption rates of 0.76 to 7.8 percent have been determined (Wrenn et al., 1985). Uranium may absorb through skin when applied in concentrated solutions (concentration level not reported); the extent of absorption appears to be dose-dependent.

\section{Tissue accumulation and clearance}

In humans exposed to background levels of uranium, the highest uranium concentrations were found in the bone, muscle, lung, liver, and kidney (Fisenne et al., 1988). Uranium retention in bone consists of a short retention half-time of 20 days, followed by a long-retention half-time of 5000 days for the remainder (Tracy et al., 1992).

In body fluids, uranium tends to be converted into water-soluble hexavalent uranium (Berlin and Rudell, 1986). Approximately 60 percent of the uranium in plasma complexes with low-molecular-weight anions (e.g., bicarbonates and citrates), while the remaining 40 percent binds to the plasma protein transferrin (Stevens et al., 1980). Following oral exposure in humans, more than 90 percent of uranium is excreted in the feces and not absorbed in the gastrointestinal tract. Of the small percentage absorbed (typically less than 5 percent), approximately 60 percent is excreted in the urine within 24 hours whereas the remainder is distributed to the skeleton and soft tissue; 98 percent of that amount is excreted within 7 days, based on animal studies (Ballou et al., 1986; Leach et al., 1984; Sullivan et al., 1986). A small portion of the absorbed uranium is retained for a longer period.

\section{Environmental sources of uranium}

Uranium is a ubiquitous element, present in the earth's crust at approximately 4 parts per million. Uranium concentrations in ground water and surface water averaged $1 \mathrm{pCi} / L$ and $3 \mathrm{pCi} / \mathrm{L}$, respectively (NCRP, 1984). It is taken up from the soil into plant tissues to an extent depending on the plant species and the depth of its root system (Berlin and Rudell, 1986). Plant concentrations of uranium averaged 0.075 micrograms per kilogram $(\mu \mathrm{g} / \mathrm{kg})$ of fresh plant material in one study (Tracy et al., 1983). 
The main dietary source of natural uranium for the general population is food such as potatoes, grains, meat, and fresh fish, which may contain uranium concentrations between 10 and $100 \mu \mathrm{g} / \mathrm{kg}$ (Prister, 1969). The total dietary uranium intake from consuming average foods is approximately $1 \mu \mathrm{g}$ per day, approximately 20 to 50 percent of which can come from drinking water. Cereals and vegetables, particularly root crops, probably contribute most to the daily uranium intake (Berlin and Rudell, 1986).

\section{Uranium toxicity}

No human deaths definitely attributable to uranium ingestion have been reported; therefore, no lethal dose (LD) has been determined for humans. A uranium dose as low as $14 \mathrm{mg} / \mathrm{kg}$-day resulted in the death of 50 percent of exposed laboratory animals following 23-day oral exposures $\left(L_{50,23}\right)$. The level of $L D_{50,23}$ depends on the solubility of the uranium compound tested (higher solubility compounds have greater toxicity), route of exposure, and animal species. High doses of uranium cause complete kidney and respiratory failure.

No chronic toxic effects have been reported in humans following oral exposure to uranium. Data available from populations occupationally exposed to high concentrations of uranium compounds through inhalation and information from studies in experimental animals indicate that the critical organ for chronic uranium toxicity is the kidney, specifically, the proximal tubule (Friberg et al., 1986). In humans, chemical injury reveals itself through increased catalase excretion in urine and proteinuria. Dose-response data for the toxic effect of uranium on the human kidney are limited.

The LOAEL of uranyl nitrate to cause moderate renal damage was given to rabbits in diet at $2.8 \mathrm{mg}$ uranium per $\mathrm{kg}$ per day (Maynard and Hodge, 1949). The EPA oral reference dose of $0.003 \mathrm{mg} / \mathrm{kg}$-day was derived based on this study (EPA, 1994a). The EPA applied an uncertaintly factor of 1000 to the LOAEL $(2.8 \mathrm{mg} / \mathrm{kg}$-day), which reflects intraspecies and interspecies variability and an uncertainty associated with the use of a LOAEL rather than the preferred use of a NOAEL. Figure 5.4 summarizes the health effects of uranium as a function of dose.

\subsection{CONTAMINANT INTERACTIONS}

Some information is available on potential interactions among contaminants found at UMTRA sites. However, discussions of potential interactions can generally be presented only qualitatively. In addition to physiological variables among individuals that can affect toxicity, uncertainties in interactions can also result from 1) differences in the relative exposure concentrations of the different contaminants compared with the concentrations tested experimentally and 2) the presence of additional ground water constituents in sufficient quantities to modify predicted toxicities even though the constituents themselves are not considered contaminants of concern for human health. Therefore, the interactions described below should be recognized as factors that can influence 


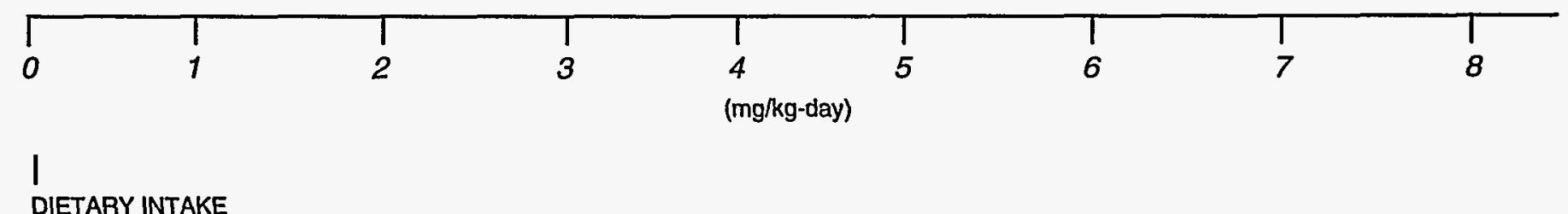

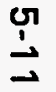

$\triangle$ oral Reference dOSE (0.003 $\mathrm{mg} / \mathrm{kg}$-day)

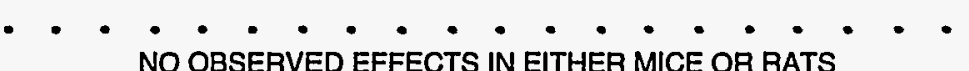

(SUFFICIENT HUMAN DATA NOT AVAILABLE)

\section{L. .}

MILD TOXICITY

(RABBITS, RENAL DAMAGE)

FIGURE 5.4

URANIUM TOXICITY RANGES 
predicted toxicity, although the precise nature and magnitude of that influence cannot be determined.

Iron and other metals influence the rate of manganese absorption. In states of iron deficiency, manganese is actively absorbed from the intestine. In states of excess iron, manganese absorption is by diffusion only (Saric, 1986). Because iron levels in ground water at the site are at naturally high levels, the amount of manganese absorbed by a person ingesting ground water at the site may be reduced. This, in turn, may reduce the potential for toxic effects to occur from manganese ingestion. Furthermore, high levels of dietary calcium and phosphorus increase the requirements for manganese in several species (Lönnerdal et al., 1987). Thus, intake of these elements may alter the potential for absorption and potential toxicity of manganese.

The primary toxicity of molybdenum is related to its interactions with copper leading to altered excretion patterns for this element. Ruminants seem to be the most susceptible species to imbalances among these elements. In ruminants, copper has been shown to prevent the accumulation of molybdenum in the liver and may antagonize molybdenum absorption. Molybdenum can produce a functional copper deficiency. The antagonism of molybdenum to copper is augmented by sulfate (Casarett and Doull, 1991). In laboratory animal studies, molybdenum toxicity is more pronounced in situations where dietary copper intake is low (EPA, 1994a).

In animal studies iron status affected the gastrointestional absorption of uranium; however, the reported results were inconclusive (EPA, 1989e). No other information was available on uranium interactions with other metals.

\subsection{CONTAMINANT RISK FACTORS}

The EPA Office of Research and Development has calculated acceptable intake values, or RfDs, for long-term (chronic) exposure to noncarcinogens. These values are estimates of route-specific exposure levels not expected to cause adverse effects when exposure occurs for a substantial portion of the lifetime. Some of the chronic RfDs are adopted as subchronic RfDs. The RfDs include safety factors to account for uncertainties associated with limitations of the toxicological data base, including extrapolating animal studies to humans and accounting for variability in response from sensitive individuals and accounting for uncertainties associated with extrapolation from a LOAEL to a NOAEL, and from shorter term or subchronic exposures to chronic exposures. These values, updated quarterly, are published in the Health Effects Assessment Summary Tables (HEAST). They are also provided through the EPA'S IRIS data base. Table 5.1 summarizes the most recent oral RfDs for the noncarcinogenic contaminants of concern.

The EPA currently classifies all radionuclides as Group A, or known human carcinogens, based on their property of emitting ionizing radiation and on the 
Table 5.1 Toxicity values: potential noncarcinogenic effects

\begin{tabular}{|c|c|c|c|c|c|}
\hline Chemical & $\begin{array}{c}\text { Chronic oral RfD } \\
\text { (mg/kg-day) }\end{array}$ & $\begin{array}{c}\text { Confidence } \\
\text { level }\end{array}$ & Critical effect/organ & $\begin{array}{l}\text { RfD basis/RfD } \\
\text { source }\end{array}$ & $\begin{array}{c}\text { Uncertainty } \\
\text { factor }\end{array}$ \\
\hline Manganese & 0.005 & $\overline{N A}$ & Central nervous system & $\begin{array}{l}\text { Water/IRIS } \\
\text { Food/IRS }^{b}\end{array}$ & $\begin{array}{l}1 \\
1\end{array}$ \\
\hline Molybdenum & 0.005 & Medium & $\begin{array}{l}\text { Increased uric acid levels in the } \\
\text { blood, decreased copper levels }\end{array}$ & Diet/IRIS ${ }^{b}$ & 30 \\
\hline $\begin{array}{l}\text { Uranium (soluble } \\
\text { salts) }\end{array}$ & 0.003 & Medium & $\begin{array}{l}\text { Nephrotoxicity, decreased body } \\
\text { weight }\end{array}$ & Diet/IRIS ${ }^{b}$ & 1000 \\
\hline
\end{tabular}

${ }^{\mathrm{a} T h e s e}$ doses are adopted as subchronic oral reference doses, except for uranium.

${ }^{b} E P A, 1994 a$.

NA - not applicable. 
evidence provided by epidemiological studies of radiation-induced cancer in humans. At sufficiently high doses, ionizing radiation acts as a complete carcinogen (both initiator and promoter), capable of increasing the probability of cancer development. However, the actual risk is difficult to estimate, particularly for the low doses encountered in the environment. Most of the reliable data were obtained under conditions of high doses delivered acutely. It is not clear whether cancer risks at lower doses are dose-proportional (i.e., the linear dose-response hypothesis) or whether the risk is greatly reduced at low doses (the threshold hypothesis) (Latarjet, 1992; Shadley and Wiencke, 1989; Rigaud et al., 1993; Lazo, 1994; Oftedal, 1989; Casarett and Doull, 1991). A conservative assumption rejects the threshold hypothesis and assumes that any dose adds to the risk of cancer.

Risk factors are published in HEAST and IRIS for correlating the intake of carcinogens over a lifetime with the increased excess cancer risk from that exposure. Table 5.2 provides the most recent cancer slope factors for the uranium-234/-238 radioactive decay series. 
Table 5.2 Toxicity values: potential carcinogenic effects

\begin{tabular}{|c|c|c|c|c|}
\hline Parameter & $\begin{array}{c}\text { Oral slope factor } \\
(\mathrm{pCi})^{-1},(\mathrm{mg} / \mathrm{kg} \text {-day })^{-1}\end{array}$ & $\begin{array}{c}\text { Weight of evidence } \\
\text { classification }\end{array}$ & Type of cancer & $\begin{array}{c}\text { Slope factor basis/slope } \\
\text { factor source }\end{array}$ \\
\hline Lead-210 & $1.01 \mathrm{E}-09$ & A & Bone & HEAST \\
\hline Polonium-210 & $3.26 E-10$ & A & Liver, kidney, spleen & HEAST \\
\hline Radium-226 ${ }^{\mathrm{b}}$ & $2.96 E-10$ & A & Bone & HEAST \\
\hline Thorium-230 & $3.75 \mathrm{E}-11$ & A & c & HEAST \\
\hline Uranium-238 ${ }^{b}$ & $6.20 E-11$ & A & d & HEAST \\
\hline Uranium-234 & $4.44 E-11$ & A & d & HEAST \\
\hline
\end{tabular}

${ }^{\text {aEPA, 1994b. }}$

${ }^{b}$ Oral SF includes the contributions from short-lived decay products, assuming equal activity concentrations (i.e., secular equilbrium) with the principle nuclide in the environment.

${ }^{c}$ Target organs systems have not been identified for oral exposure to thorium.

${ }^{d}$ No human or animal studies have shown a definite association between oral exposure to uranium and development of cancer.

A - known human carcinogen.

SF - slope factor.

HEAST - Health Effects Assessment Summary Tables. 



\subsection{HUMAN HEALTH RISK EVALUATION}

To evaluate human health risks to an individual or population, the results of the exposure assessment and toxicity assessment are combined. As discussed in Section 5.0, potential adverse health effects are a function of how much of the contaminant an individual takes into his or her body. At lower levels, many of the contaminants associated with the mill tailings are essential nutrients that are beneficial to health. At higher levels, these same elements can cause adverse health effects.

In this section, expected intake ranges for possible ground water ingestion from the unconsolidated material is correlated to potential health effects.

\subsection{POTENTIAL NONCARCINOGENIC HEALTH EFFECTS}

\subsubsection{Ground water}

The potential for noncarcinogenic health effects was evaluated for the 1- to 10-year-old child. Children were evaluated for noncarcinogenic effects because they have a higher intake-to-body weight ratio than adults and, thus, would be expected to receive a larger daily dose than adults. Potential noncarcinogenic effects were assessed for the following chemical contaminants of potential concern: manganese, molybdenum, and uranium.

As shown in Figure 6.1, potential adverse health effects may result from manganese intake if ground water from the unconsolidated material in Area $C$ is used for drinking. More than 99 percent of the exposure distribution falls above the EPA oral RfD derived from drinking water consumption studies and above the threshold of mild neurological symptoms in adults. In addition, approximately 15 percent of the intakes exceeded $0.4 \mathrm{mg} / \mathrm{kg}$-day, which is an estimated drinking water intake associated with symptoms of intoxication, including a mask-like face, muscle rigidity and tremors, and mental disturbances. Two cases of death were reported in the literature among the individuals ingesting manganese at this level. For comparison purposes, child exposure to manganese was calculated using the maximum detected value $(4.05 \mathrm{mg} / \mathrm{L})$ in background well 410. As shown in Figure 6.1, the estimated average exposure dose is $0.12 \mathrm{mg} / \mathrm{kg}$-day, which falls within the range of early neurological symptoms. This estimate, however, is based on a maximum detection from only one upgradient well and may not be representative of long-term background conditions.

Molybdenum levels in ground water from the unconsolidated material in Area $C$ are not expected to result in any adverse health effects, as shown in Figure 6.2. All estimated intake levels fall within nutritional or dietary intake levels. The estimated intakes are also well below the EPA oral RfD. 


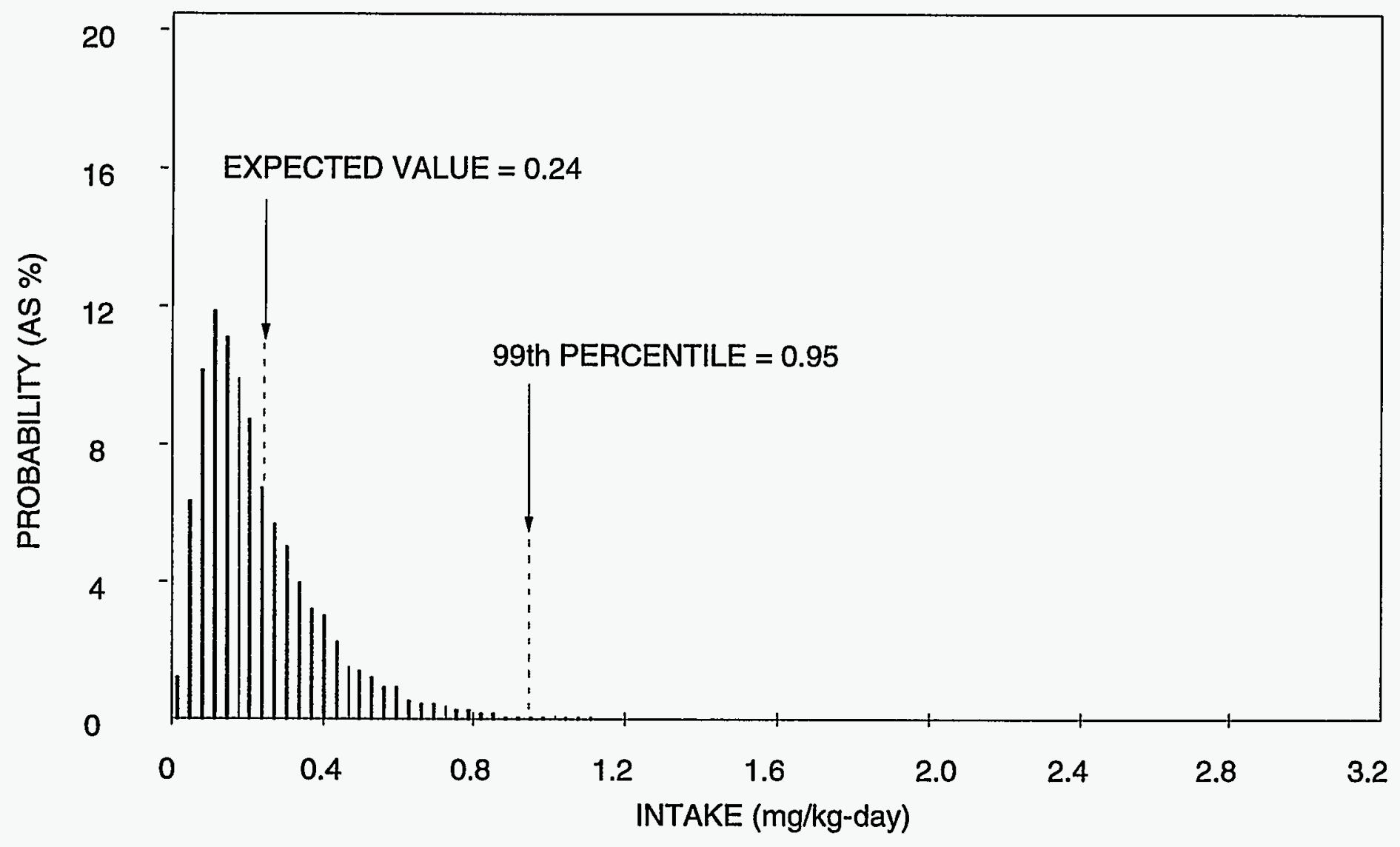

$\triangle$ BACKGROUND DRINKING WATER INTAKE $(0.003 \mathrm{mg} / \mathrm{kg}$-day)

$\sqcup$ DIETARY INTAKE FROM FOOD

$\triangle$ EPA RID - DRINKING WATER (0.005 mg/kg-day)

....... EARLY NEUROLOGICAL SYMPTOMS

PARKINSON-LIKE EFFECTS

FIGURE 6.1

HEALTH EFFECTS OF POTENTIAL MANGANESE EXPOSURE RANGES TO CHILDREN

CANONSBURG, PENNSYLVANIA, SITE 


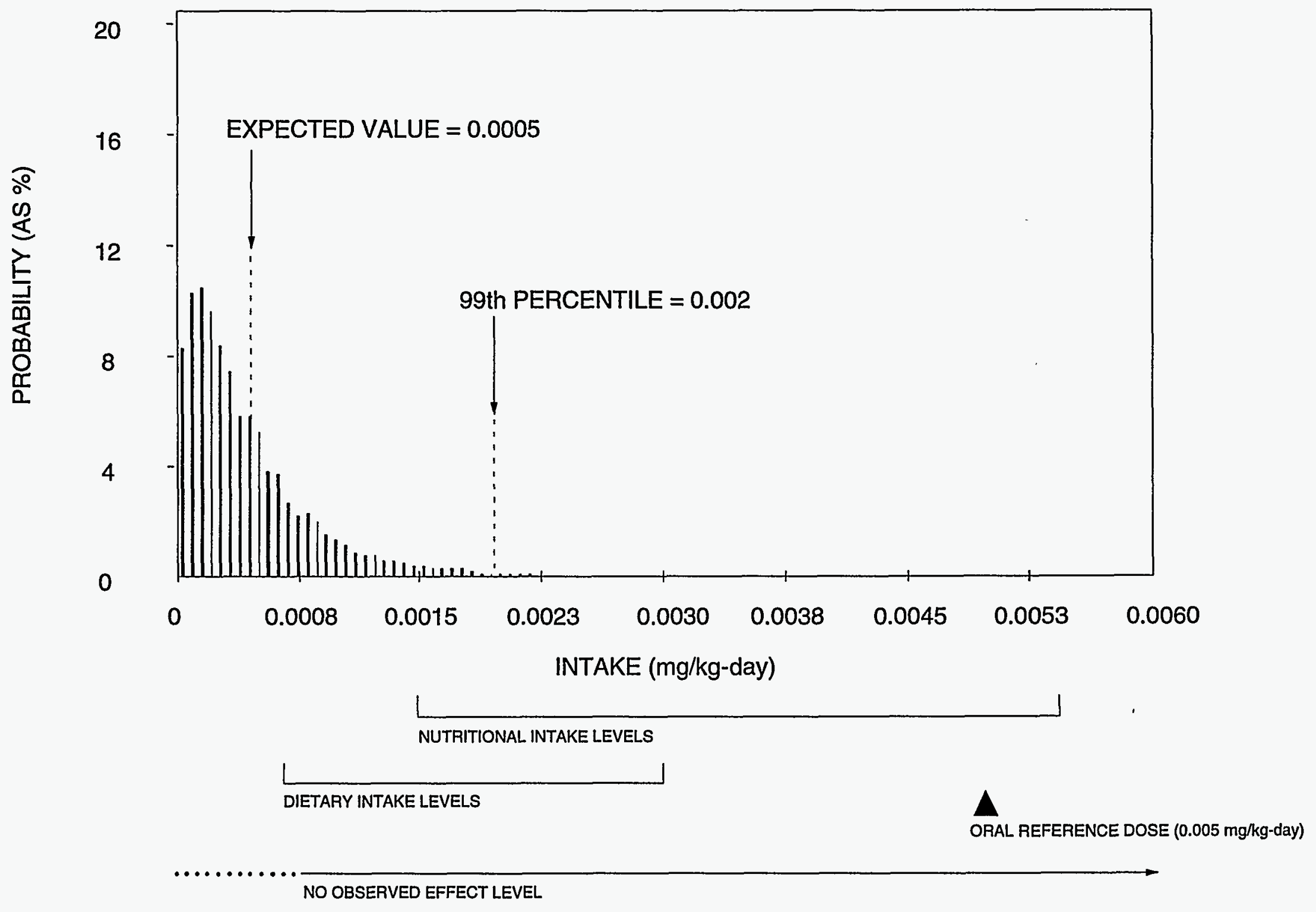

FIGURE 6.2

HEALTH EFFECTS OF POTENTIAL MOLYBDENUM EXPOSURE RANGE TO CHILDREN CANONSBURG, PENNSYLVANIA, SITE 
As shown in Figure 6.3, only 1 percent of the exposure range for uranium exceeds the EPA oral RfD. The estimated intakes for uranium fall well below any toxic effects observed in humans or in animal studies.

\subsubsection{Surface water/sediment}

Potential noncarcinogenic health effects were evaluated for 6-to 12-year-old children who may be exposed to contaminants while playing in Chartiers Creek. Doses were estimated in Section 4.0 for incidental surface water ingestion, dermal contact with surface water, and incidental sediment ingestion. Doses were also estimated for adults who may ingest fish caught from Chartiers Creek. These doses are presented again in Table 6.1 for comparison with relevant toxicity data from Section 5.0. As seen in Table 6.1, the estimated doses for a child playing in the creek are at levels that would not be expected to result in adverse effects. All doses for the child fall below the EPA oral RfD and are below dietary and nutritional intake levels. Also, the estimated manganese dose for adults from fish ingestion is well below the EPA oral RfD for manganese in food sources.

\subsection{POTENTIAL CARCINOGENIC HEALTH EFFECTS}

The contaminants considered for potential carcinogenic risk include uranium as well as all uranium isotopes. These constituents are radioactive and, as such, are considered potential carcinogens. Figure 6.4 shows the exposure distribution for uranium-234 and uranium-238 ground water exposure doses and their potential lifetime carcinogenic risks. Although natural uranium has not been demonstrated to cause cancer in humans or animals following ingestion exposures, these estimates of excess lifetime cancer risk are based on the cancer slope factor developed by the EPA. The majority of the exposure distribution for ground water ingestion of uranium falls within the range of 1 in a million (1E-06) to 1 in 100,000 (1E-05). This falls within the National Contingency Plan (NCP) guidance for acceptable excess risk of 1 in a million (1E-06) to 1 in 10,000 (1E-04). The expected exposure dose results in an excess lifetime cancer risk of 9 in a million, and the upper 99th percentile exposure is expected to result in an excess lifetime cancer risk of 3 in 100,000. The distribution presented here is considered conservative because it is based on a cumulative 30-year exposure duration. As discussed previously, this exposure duration is probably appropriate, but ground water uranium concentrations resulting from uranium processing at this site are expected to decline after the tailings are stabilized; therefore, this exposure distribution may overestimate risk.

Uranium is the only radionuclide statistically above background in the unconsolidated material ground water at the Canonsburg site. However, because uranium decays to radioactive progeny, longer-lived radioactive progeny of the uranium decay series were evaluated for carcinogenic risk. Thus, the carcinogenic risk of radium-226, lead-210, polonium-210, and thorium-230 


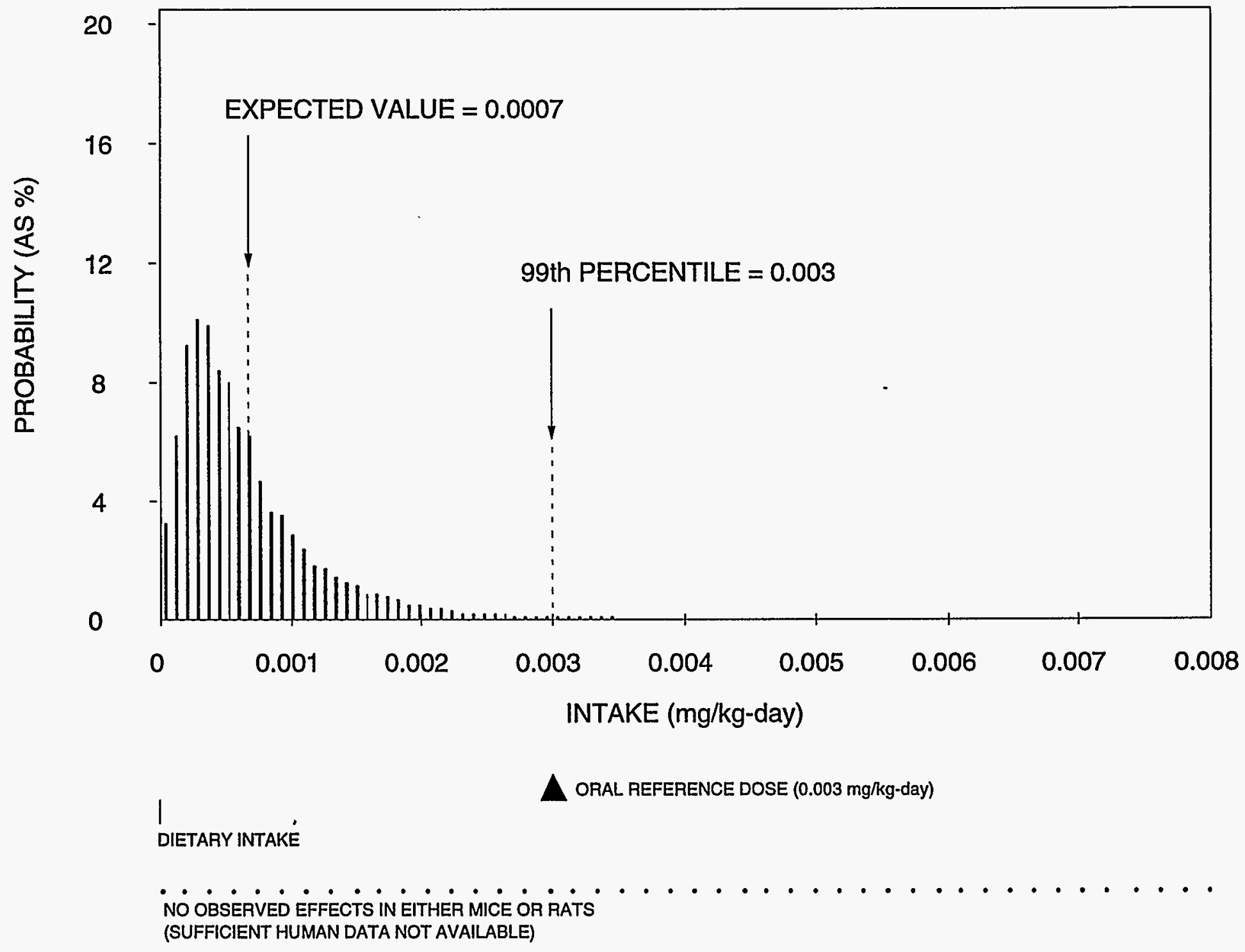

FIGURE 6.3

HEALTH EFFECTS OF POTENTIAL URANIUM EXPOSURE RANGES TO CHILDREN CANONSBURG, PENNSYLVANIA, SITE 
Table 6.1 Estimated doses and toxicity values for the surface water and sediment exposure pathways in Chartiers Creek, Canonsburg, Pennsylvania, site

\begin{tabular}{|c|c|c|c|c|c|c|}
\hline \multirow[b]{2}{*}{$\begin{array}{c}\text { Contaminant } \\
\text { of potential } \\
\text { concern }\end{array}$} & \multicolumn{4}{|c|}{ Exposure doses (mg/kg-day) } & \multicolumn{2}{|c|}{$\begin{array}{l}\text { Toxicity values } \\
\text { (mg/kg-day) }\end{array}$} \\
\hline & $\begin{array}{c}\text { Surface } \\
\text { water } \\
\text { ingestion }\end{array}$ & $\begin{array}{c}\text { Dermal contact } \\
\text { with surface } \\
\text { water }\end{array}$ & $\begin{array}{l}\text { Sediment } \\
\text { ingestion }\end{array}$ & $\begin{array}{c}\text { Fish } \\
\text { ingestion }\end{array}$ & $\begin{array}{l}\text { EPA } \\
\text { oral } \\
\text { RfD }\end{array}$ & $\begin{array}{c}\text { Dietary intake } \\
\text { level }\end{array}$ \\
\hline Manganese & 0.00005 & 0.00001 & 0.002 & 0.009 & $\begin{array}{l}0.005^{a} \\
0.14^{b}\end{array}$ & NA \\
\hline Molybdenum & 0.00005 & 0.00001 & 0.00006 & NC & 0.005 & $0.0007-0.003$ \\
\hline \multicolumn{7}{|c|}{$\begin{array}{l}\text { aEPA oral RfD for manganese in water. } \\
\text { bEPA oral RfD for manganese in food. }\end{array}$} \\
\hline
\end{tabular}

were evaluated, based on ground water ingestion from the unconsolidated material in Area C. As shown in Table 6.2, the estimated increased carcinogenic risk from these radionuclides is about 1 in 10,000 (1E-04). This risk level is at the upper end of the acceptable range of the 1E-06 to $1 \mathrm{E}-04$ as recommended in the NCP for Superfund sites. If the cancer risk estimated for these radionuclides is added to the range of carcinogenic risk estimated for uranium, the total excess cancer risk could also be at the upper end of the NCP risk range.

\subsection{LIMITATIONS OF THE HUMAN HEALTH RISK EVALUATION}

The following potential limitations apply to the interpretation of this human health risk evaluation:

- Subpopulations with increased sensitivity are not specifically addressed on the graphs. Expected sensitivities in certain groups were discussed in the text to the extent possible.

- Some individuals may be more sensitive to the toxic effects of certain constituents for undetermined reasons; therefore, adverse health effects may occur in sensitive individuals at lower exposure levels.

- Data available to interpret potential adverse health effects may not always be sufficient to allow accurate determination of all health effects due to lack of testing in humans or testing of dose ranges other than those expected at the site. 


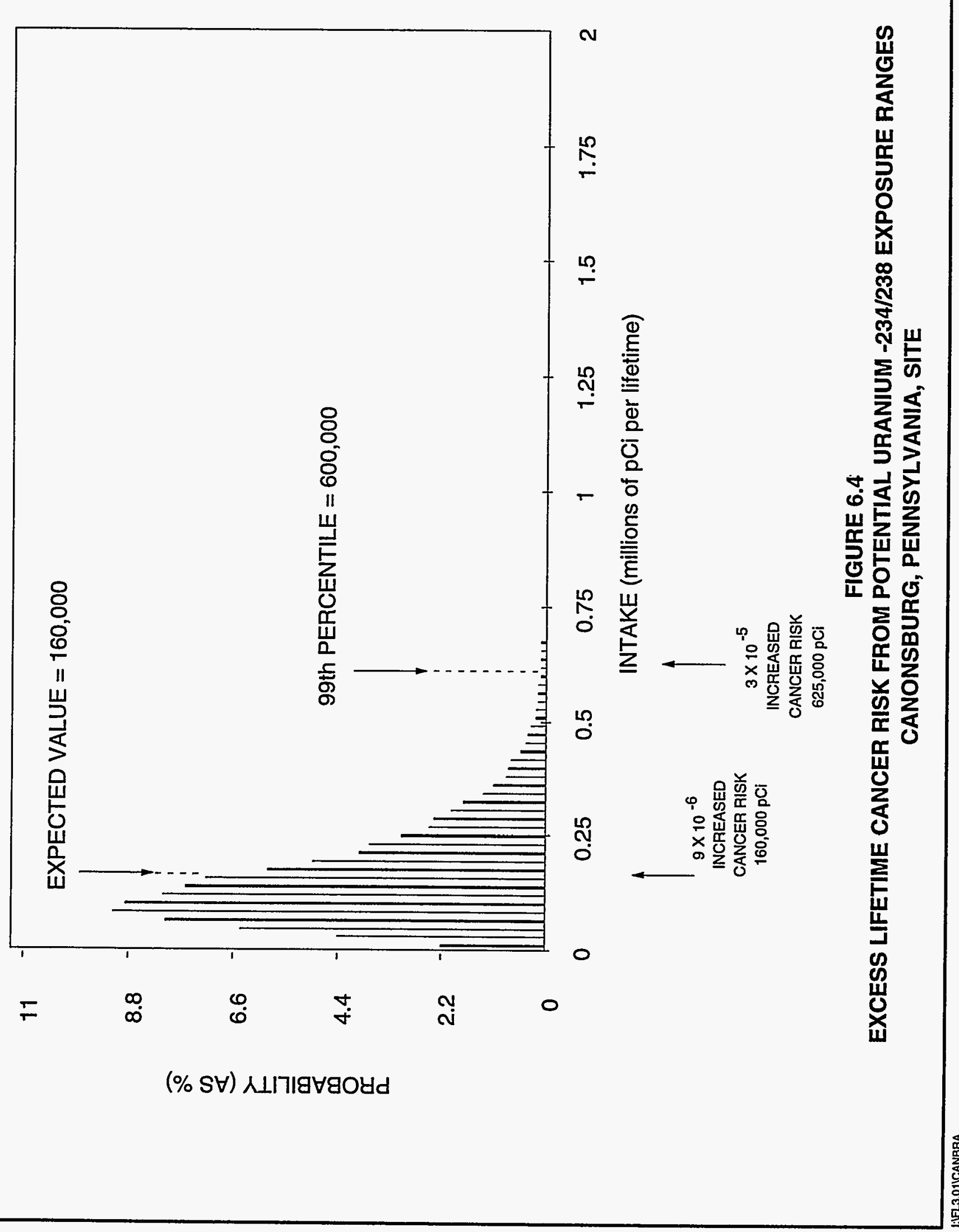


Table 6.2 Carcinogenic risk for the unconsolidated layer ground water ingestion pathway, Canonsburg, Pennsylvania, site

\begin{tabular}{lcccc}
\hline $\begin{array}{l}\text { Contaminant of } \\
\text { potential concern }\end{array}$ & $\begin{array}{c}\text { Exposure point } \\
\text { concentration } \\
(\mathbf{p C i} / \mathrm{L})\end{array}$ & $\begin{array}{c}\text { Intake } \\
\text { (pCi per lifetime) }\end{array}$ & $\begin{array}{c}\text { Oral slope } \\
\text { factor } \\
(\mathbf{p C i})^{-1}\end{array}$ & Lifetime risk \\
\hline $\begin{array}{l}\text { Radionuclides } \\
\text { Lead-210 }\end{array}$ & $5.0^{\mathrm{a}}$ & 105,000 & $1.01 \mathrm{E}-09$ & $1 \mathrm{E}-04$ \\
Polonium-210 & $0.2^{\mathrm{a}}$ & 4,200 & $3.20 \mathrm{E}-10$ & $1 \mathrm{E}-06$ \\
Radium-226 & $0.7^{\mathrm{b}}$ & 14,700 & $2.96 \mathrm{E}-10$ & $4 \mathrm{E}-06$ \\
Thorium-230 & $0.7^{\mathrm{a}}$ & 14,700 & $3.75 \mathrm{E}-11$ & $6 \mathrm{E}-07$ \\
& & & Total: & $1 \mathrm{E}-04$ \\
\hline
\end{tabular}

aaximum observed concentration in filtered or unfiltered water samples, 1986-1993.

${ }^{b}$ Maximum observed concentration in filtered or unfiltered water samples, 1988-1993.

- Although the areal extent of ground water contamination and its movement have been evaluated hydrologically and geochemically, the monitoring locations sampled may not include the most contaminated areas. In addition, concentrations may decrease substantially over time.

- The results of risk evaluation presented in this document are based on filtered (0.45-micron) ground water samples. Therefore, the potential loss of certain ground water constituents as a consequence of filtration represents a source of uncertainty.

- Only the drinking water exposure pathway has been considered in depth, although other pathways have been screened to determine their relative and independent contribution. However, the incremental or independent contribution from the ground water-irrigated produce ingestion pathway could not be estimated here.

- This risk assessment evaluates only risks related to inorganic ground water contamination. Potential contamination from any of the organic constituents that might be used in uranium processing has not been addressed.

The evaluation presented here has considered these limitations and compensated whenever possible by presenting toxicity ranges and probabilistic exposure assessments rather than point estimates to incorporate as much variability as could reasonably be defined. Section 8.2 details the impact of these potential limitations. 


\subsection{ENVIRONMENTAL EVALUATION}

The objective of the environmental portion of the risk assessment is to determine whether contaminants detected at the site have the potential to adversely affect the existing biological community at or surrounding the site. Currently, the EPA has no guidance for quantifying potential impacts to ecological receptors, but has developed a qualitative approach generally used for ecological evaluation (EPA, 1989c). With the qualitative approach, the EPA recommends comparing ambient environmental media concentrations with water quality, sediment quality, or other relevant criteria or guidelines to determine whether the concentrations that the ecological receptors are expected to encounter exceed these values.

Although the effects of contaminants on ecological receptors are a concern, it is difficult to predict whether effects on individual populations will cause damage to the community or ecosystem. If a prey species is affected, predators may be able to feed on other species; losses of predators may be compensated for by other predators or by immigration of another predator population. Thus, it may be difficult to recognize whether adverse effects are occurring, unless there are gross impacts such as mass killings within a population or community. Evidence of gross impacts were not observed during the field survey at the Canonsburg site.

Sublethal effects may occur if contaminants are present at concentrations that may not kill organisms directly, but diminish their ability to survive or reproduce. Types of sublethal effects include behavioral changes, reduced reproductive success, or enzyme level changes, which can affect the population or community level of organization. However, it is often difficult to identify and measure the sublethal effects and establish a causal relationship to a specific environmental stressor, such as a chemical contaminant.

Thus, it is important to note that this preliminary ecological risk assessment cannot account for all potential variables. However, it does provide a qualitative evaluation of potential ecological risks at this site.

\subsection{EXPOSURE CHARACTERIZATION}

This section identifies the potential exposure pathways associated with the Canonsburg site. For risk to exist, a receptor must be exposed to contaminants. Exposure can occur only with both a source of contamination and a mechanism of transport to a receptor population or individual.

Ground water, and potentially surface water and sediment, are the only possibly impacted media at the site. Thus, soil or air exposure pathways (such as incidental ingestion of soil, dermal contact with soil, and inhalation of air containing particulates) do not represent an ecological concern and will not be evaluated in this baseline risk assessment. However, surface water-related or ground water-related exposure pathways (such as ingestion of surface water potentially affected by contaminated ground water, bioconcentration of 
contaminants in surface water by aquatic organisms, and consumption of organisms that have accumulated contaminants) are possible at the site.

The main surface water body in the Canonsburg site area is Chartiers Creek (Figure 7.1). The site lies in the Chartiers Creek basin along the creek's southern bank, approximately $15 \mathrm{mi}(24 \mathrm{~km})$ upstream of its confluence with the Ohio River. In the Canonsburg area, Chartiers Creek is a meandering stream 75 to $100 \mathrm{ft}(23$ to $30 \mathrm{~m})$ wide and approximately $10 \mathrm{ft}(3 \mathrm{~m})$ deep (DOE, 1983a). In the site vicinity, the stream dimensions are usually much less, ranging from approximately 25 to $40 \mathrm{ft}(7.6$ to $12 \mathrm{~m})$ wide and approximately $6 \mathrm{ft}(1.8 \mathrm{~m})$ deep.

This water body is a potential exposure point for resident aquatic life and terrestrial wildlife (as well as domestic animals) that may contact surface water and/or sediments. This risk assessment evaluates these exposure pathways.

Another potential current pathway could involve plant uptake of contaminants in ground water. Due to the shallow depth to ground water lapproximately $5 \mathrm{ft}$ [1.5 $\mathrm{m}]$ or less below land surface), plant roots may reach contaminated ground water. This risk assessment evaluates plant uptake, assuming that the plant roots reach soil saturated with ground water.

Another potential pathway involves use of ground water as a water source for irrigating garden plants. For this baseline risk assessment, it was assumed that a domestic well, at some point in the future, could be installed in Area C, which is the site area being considered for public use. The water from this hypothetical well could be used for irrigating garden plants.

\subsection{ECOLOGICAL RECEPTORS}

This section identifies the ecological resources present at the site and vicinity that potentially may be exposed to site-related contaminants.

The following information on ecological receptors is based primarily on surveys performed before the tailings encapsulation process was initiated and is provided as a historical perspective. Limited observations of aquatic organisms (at the surface water and sediment sampling locations) and of terrestrial flora and fauna were made during a 19 and 20 October 1993 field survey.

\subsubsection{Terrestrial resources}

The Canonsburg disposal cell and surrounding land was planted with grass after the completion of remedial action. The disposal cell and surrounding land within the site fence line is now covered with a dense growth of grass, as well as occasional herbaceous species such as yellow hop clover, red clover, yarrow, Queen Anne's lace, curled dock, and alfalfa. This land is mowed once or twice a year which serves to maintain the grass and herb cover; very few woody plant species occur in this mowed area (TAC, 1990; 1993). 


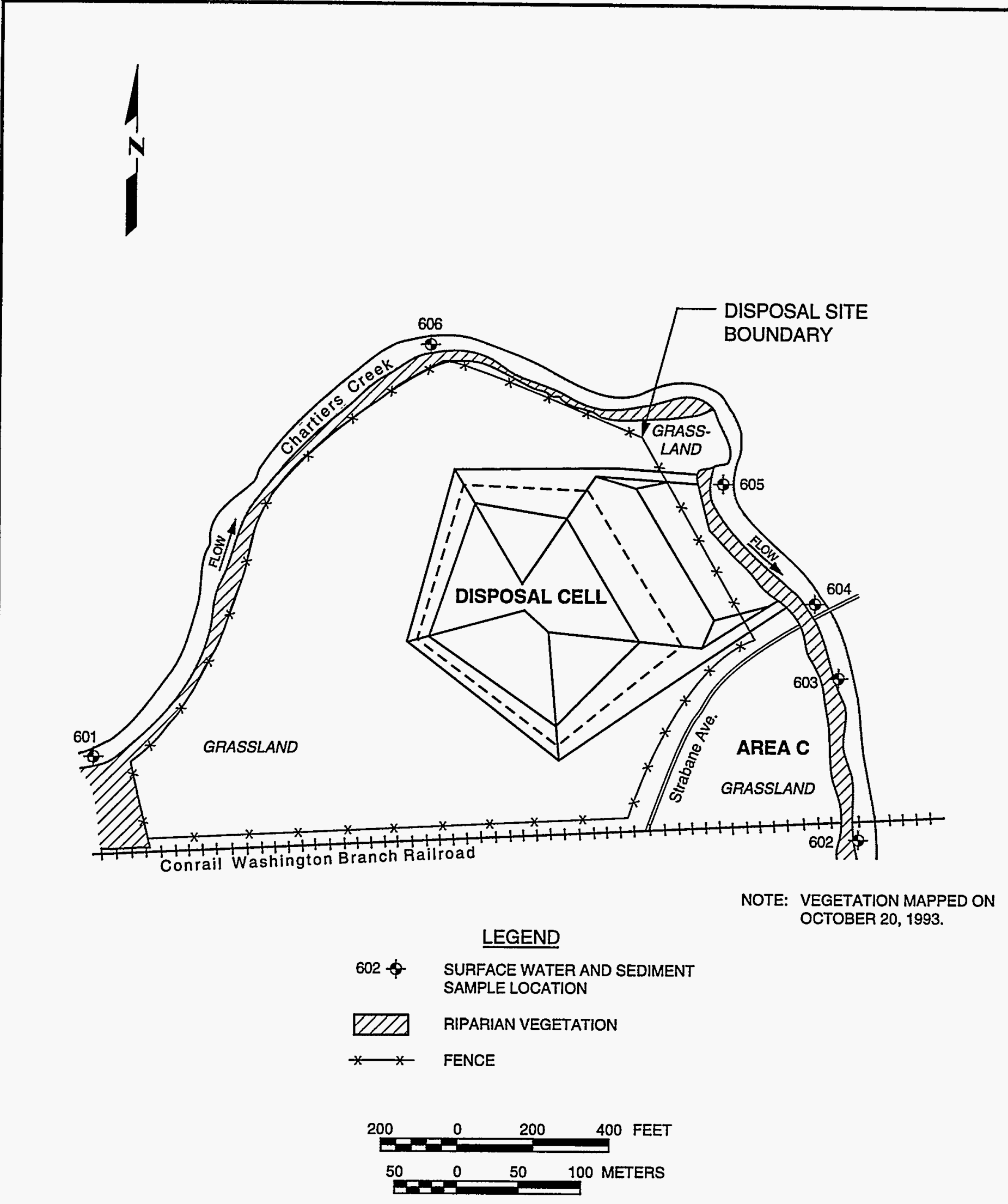


Chartiers Creek borders the site on the north, west, and east and a wooded riparian zone occurs between the site fence line and the creek (Figure 7.1). This vegetation grows in a thin band along the eastern site boundary and forms a wider band on the north and west sides of the site. The wooded riparian plant community on the north and west sides of the site occurs on a bank 3 to $15 \mathrm{ft}$ $(1$ to $5 \mathrm{~m}$ ) above the creek. The majority of the site is covered by a grassdominated plant community, which typically grows up to the creek bank.

Within the riparian community along the creek, sycamore was the most frequently observed canopy tree. A few large black willows were also observed. Other large trees observed in lesser numbers were box elder, basswood, black locust, and silver maple. Box elder was clearly the most common understory species observed. Other species noted in the understory were basswood, sugar maple, black cherry, red oak, black locust, and apple. Wild grape vines were commonly observed growing in the trees. Ground cover was dominated by herbaceous species. Some grass and Carex sp. were observed. Black snakeroot was also very common, along with poison ivy, Aster sp., and Rubus sp. (TAC, 1993).

Only limited surveys for wildlife have occurred at or near the Canonsburg site. No amphibians and only one reptile (garter snake) were observed during site visits, although a variety of frog, toad, salamander, and snake species are expected to occur at the site. Surveys for nesting birds were not conducted; species recorded in the fall include the blue jay, song sparrow, crow, downy woodpecker, black capped chickadee, white-breasted nuthatch, robin, and Carolina wren. Birds of prey recorded were the kestrel and Coopers Hawk. Waterfowl such as the mallard and wood duck have been observed in the creek, as well as the green heron. Woodchuck were observed in both the grassland and wooded riparian plant communities (DOE, 1983a; TAC, 1993).

\subsubsection{Aquatic organisms}

No quantitative surveys of aquatic organisms occurring in Chartiers Creek have been conducted to date as part of the technical assistance contractor (TAC) investigation (DOE, 1994). A brief qualitative survey of the aquatic organisms in Chartiers Creek was conducted in the vicinity of the six surface water and sediment sampling locations (Figure 7.1). The bottom sediments were composed of cobble and silts. Several pools and riffles occur in the reach of Chartiers Creek in the site vicinity.

A fine-mesh dip-net and soil sieve were used to collect fish and benthic macroinvertebrates at each of the surface water sampling locations. One fish, a darter (Etheostomata), was collected in a riffle area near location 604. Several unidentified fish were observed at various points along the creek. Four crayfish were collected and numerous burrows and chimneys were observed along the stream. Numerous isopods were observed on the undersides of rocks in the riffle areas. Chironomids and oligochaetes were found in surficial sediments throughout the creek. Limpets were observed in the riffle areas attached to 
attached to rocks. No apparent differences among the locations were noted during the October 1993 survey with respect to the fauna observed.

Based on previous investigations in the site vicinity, fish reported in the reach of Chartiers Creek include creek chub, white sucker, striped shiner, and carp (DOE, 1983a). Also, the following benthic macroinvertebrates were collected from the creek during previous investigations: oligochaetes, chironomids, leeches, snails, water beetles, isopods, and crayfish (DOE, 1983a).

\subsection{CONTAMINANTS OF ECOLOGICAL CONCERN}

\subsubsection{Ground water}

The list of ground water contaminants exceeding background levels in the unconsolidated unit was used as the list of contaminants of potential concern for evaluating potential plant receptors exposed to ground water (see Section 3.3). This list was developed using data from the most contaminated monitor wells finished in the unconsolidated materials at the site 1412,413 , and 414). Wells 412 and 413 are downgradient of the tailings encapsulation area, and well 414 is in Area C. The list of contaminants of potential concern from these wells include the following inorganics: ammonium, arsenic, boron, calcium, chloride, magnesium, manganese, molybdenum, potassium, sodium, strontium, sulfate, and uranium. These contaminants and their associated concentrations differ from the list used for the human health risk assessment, which only assessed data from well 414, because plant roots can access ground water beneath the site at all locations. Thus, additional contaminants of concern were added to those evaluated in the human health assessment based on the additional consideration of data from wells 412 and 413.

The list of ground water contaminants exceeding background levels in the unconsolidated unit was also used as the list of contaminants of potential concern for evaluating potential receptors of irrigation water. However, the irrigation pathway only considered data from well 414, which is in Area C. As in the human health assessment, Area $C$ was assumed to be the only area of the site potentially accessible for the installation of a well and subsequent use for irrigation. The first column of Table 3.3 provides the list of contaminants of potential concern from well 414: ammonium, boron, calcium, manganese, molybdenum, potassium, strontium, and uranium.

The exposure point concentrations used for ground water were either the upper 95 percent confidence limit of the concentration from any one well or the maximum detected value, whichever value was lower. When applicable, the well with the highest concentration for any one contaminant was used to represent the exposure point concentration for that contaminant. 


\subsubsection{Surface water/sediment}

The list of contaminants of potential concern in the surface water bodies was developed from the same list of contaminants evaluated for ground water because any site-related contaminants in surface water are primarily assumed to be a result of ground water discharge. This list of contaminants was then compared with the surface water data to determine which contaminants are potentially site-related (i.e., those contaminants exceeding ground water background levels in wells 412,413 , and 414). Table 7.1 presents the surface water data for these site-related contaminants.

If a contaminant was never detected in Chartiers Creek (e.g., uranium) or the concentration detected adjacent to or downstream of the site was less than or equal to the concentration upstream of the site (e.g., ammonium, sodium, sulfate, and magnesium), it was excluded as a contaminant of potential concern for ecological receptors. Additionally, calcium, chloride, and potassium were excluded as contaminants of potential concern because the differences between the upstream and adjacent concentrations were minimal (less than 5 percent). Although arsenic, boron, and strontium were identified as statistically elevated above background in ground water from monitor wells 412,413 , and 414 , no data are available for Chartiers Creek because the surface water has never been analyzed for these constituents. Based on these comparisons, manganese and molybdenum are the contaminants of potential ecological concern selected for surface waters in Chartiers Creek.

The concentrations used in these comparisons to background were from unfiltered samples. Data from unfiltered samples were used because most of the state of Pennsylvania's water quality criteria are stated as total recoverable (unfiltered) metal concentrations.

No sediment samples were collected from Chartiers Creek prior to the October 1993 sampling. The constituents analyzed in the sediments that were identified as being site-related (i.e., above background ground water quality) were evaluated in this assessment. These include the metals manganese, molybdenum, and uranium. Table 7.2 presents the sediment data for these metals by location.

\subsection{POTENTIAL IMPACTS TO WILDLIFE AND PLANTS}

\subsubsection{Terrestrial risk}

A number of potential exposure pathways were evaluated for terrestrial vegetation and wildlife. Terrestrial vegetation can be directly exposed to contaminants in ground water through uptake by the roots. Contaminants may bioaccumulate in various plant parts and exert a wide range of influences depending on the specific contaminant. Plant uptake rates vary greatly among species and are affected by soil characteristics $(\mathrm{pH}$, moisture, redox potential, organic matter, etc.), plant sensitivity, and input-output balance. Another 
Table 7.1 Occurrence of constituents detected in Chartiers Creek surface water, Canonsburg, Pennsylvania, site vicinity

\begin{tabular}{|c|c|c|c|c|c|c|}
\hline \multirow[b]{2}{*}{ Constituent } & \multirow{2}{*}{$\begin{array}{c}\begin{array}{c}\text { Upstream of } \\
\text { site } \\
\text { Location ID }\end{array} \\
601\end{array}$} & \multicolumn{4}{|c|}{$\begin{array}{c}\text { Adjacent to site }^{a} \\
\text { Location ID }\end{array}$} & \multirow{2}{*}{$\begin{array}{c}\begin{array}{c}\text { Downstream } \\
\text { of site } \\
\text { Location ID }\end{array} \\
603\end{array}$} \\
\hline & & 606 & 605 & 604 & 602 & \\
\hline Ammonium $^{b}$ & 0.25 & NA & NA & NA & 0.20 & NA \\
\hline Calcium $^{b}$ & 83 & NA & NA & NA & 87 & NA \\
\hline Chloride $^{b}$ & 50 & NA & NA & NA & 51 & NA \\
\hline Magnesium ${ }^{b}$ & 19 & NA & NA & NA & 19 & NA \\
\hline Manganese & $\begin{array}{l}0.13 \\
(0.16)^{b}\end{array}$ & 0.17 & 0.16 & 0.13 & $\begin{array}{c}0.12 \\
(0.16)^{b}\end{array}$ & 0.12 \\
\hline Molybdenum & $\begin{array}{l}0.09 \\
(0.08)^{b}\end{array}$ & 0.17 & 0.16 & 0.10 & $\begin{array}{l}0.09 \\
(0.085)^{b}\end{array}$ & 0.10 \\
\hline Potassium $^{b}$ & 6.5 & NA & NA & NA & 6.8 & NA \\
\hline Sodium $^{b}$ & 62 & NA & NA & NA & 58 & NA \\
\hline Sulfate & $280(207)^{b}$ & 250 & 250 & 280 & $270(203)^{b}$ & 270 \\
\hline Uranium & $\begin{array}{l}<0.001 \\
(<0.001)^{b}\end{array}$ & $<0.001$ & $<0.001$ & $<0.001$ & $\begin{array}{l}<0.001 \\
(<0.001)^{b}\end{array}$ & $<0.001$ \\
\hline
\end{tabular}

${ }^{\mathrm{a}}$ Refers to sampling locations adjacent to the disposal cell or Area C.

${ }^{b}$ Concentrations presented are median concentrations for unfiltered data collected from 1991 through 1993.

Note: All concentrations reported in milligrams per liter. Unfiltered data collected on 19 and 20 October 1993, unless otherwise specified.

NA - not analyzed. 
Table 7.2 Occurrence of constituents detected in Chartiers Creek sediment, Canonsburg, Pennsylvania, site vicinity

\begin{tabular}{|c|c|c|c|c|c|c|}
\hline \multirow[b]{2}{*}{ Constituent } & \multirow{2}{*}{$\begin{array}{c}\begin{array}{c}\text { Upstream } \\
\text { of site } \\
\text { Location ID }\end{array} \\
601\end{array}$} & \multicolumn{4}{|c|}{$\begin{array}{c}\text { Adjacent to site } \\
\text { Location ID }\end{array}$} & \multirow{2}{*}{$\begin{array}{c}\begin{array}{c}\text { Downstream } \\
\text { of site } \\
\text { Location ID }\end{array} \\
603\end{array}$} \\
\hline & & 606 & 605 & 604 & 602 & \\
\hline Manganese & 1410 & 1550 & 2150 & 2820 & 590 & 1900 \\
\hline Molybdenum & 33 & 13 & 96 & 25 & 2 & 16 \\
\hline Uranium & 2.0 & 1.8 & 2.0 & 2.6 & 1.6 & 2.4 \\
\hline
\end{tabular}

${ }^{a}$ Refers to sampling locations adjacent to the disposal cell or Area C.

Note: All concentrations reported in milligrams per kilogram dry weight. Data collected on 19 and 20 October 1993.

potential exposure route for plants is through garden irrigation by ground water. Foraging wildlife can be indirectly exposed to contaminants in ground water by ingesting plants or fruits (such as apples) that may have bioaccumulated certain contaminants. Terrestrial wildlife can also be exposed to contaminants in surface water bodies by ingesting the surface water, aquatic organisms that have accumulated contaminants, and/or sediments.

\section{Vegetation}

Based on the shallow depth to contaminated ground water at the site, some plants could have rooting zones in soils that intercept contaminated ground water. Concentrations of the contaminants of potential concern in plant tissue at harvestable maturity, based on uptake from ground water, could not be estimated but should be considered in future studies at the site.

Another potential exposure route for plants is through garden irrigation by ground water. Table 7.3 compares the ground water concentrations (represented by well 414 ) with the approximate concentrations in water that should be protective of plants if used as a continuous source for irrigation (EPA, 1972). The EPA developed these approximate irrigation water concentrations to protect agricultural crops from toxicity associated with buildup of a particular constituent in the soil. Two of the contaminants of potential concern, boron and manganese, have comparison values. The ground water concentration for manganese exceeds the comparison value, while boron is below the comparison value. No comparison values are available for the remainder of the contaminants of potential concern. Thus, it is not possible to evaluate the potential for these compounds to adversely affect plants when applied in irrigation water. However, based on the available information, use of the alluvial ground water near the site as a continuous source of irrigation water may result in deleterious effects to plants, due to the elevated concentration of manganese. 
Table 7.3 Comparison of contaminants of potential concern in ground water with available water quality values, Canonsburg, Pennsylvania, site

\begin{tabular}{|c|c|c|c|c|}
\hline Contaminant & $\begin{array}{l}\text { Concentration } \\
\text { in ground water }\end{array}$ & $\begin{array}{c}\text { Aquatic life } \\
\text { water quality } \\
\text { value }^{\mathbf{a}}\end{array}$ & $\begin{array}{c}\text { Water } \\
\text { concentration } \\
\text { protective of } \\
\text { livestock }^{\mathbf{b}}\end{array}$ & $\begin{array}{c}\text { Concentration } \\
\text { in irrigation } \\
\text { water } \\
\text { protective of } \\
\text { plants }^{\text {b }}\end{array}$ \\
\hline Ammonium & 1.3 & NA & NA & NA \\
\hline Boron & 0.39 & 1.6 & 5.0 & 0.75 \\
\hline Calcium & 93 & NA & NA & NA \\
\hline Manganese & 11 & 1.5 & NA & 0.20 \\
\hline Molybdenum & 0.03 & $50(0.79)^{d}$ & $10^{f}$ & NA \\
\hline Potassium & 4.3 & NA & NA & NA \\
\hline Strontium & 0.30 & NA & NA & NA \\
\hline Uranium & 0.04 & $8^{e}$ & NA & NA \\
\hline
\end{tabular}

${ }^{\mathrm{a}}$ Value obtained from the commonwealth of Pennsylvania, Title 25, Environmental Resources, Chapter 16.51, Water Quality Criteria for Toxic Substances, Pennsylvania Department of Environmental Resources (PADER, 1991), unless specified otherwise. These values are standards protective of aquatic life via chronic exposure.

${ }^{b}$ From EPA (1972), unless specified otherwise. Irrigation water values shown are for water used continuously on all soils.

"No state or federal criteria available. Value shown is the lower end of the tolerance range for freshwater organisms (EPA, 1986).

${ }^{d}$ No state or federal criteria available. Value presented is the current molybdenum criterion recommended by the U.S. Fish and Wildlife Service for the protection of aquatic organisms $(50 \mathrm{mg} / \mathrm{L})$, with one exception. The exception is for newly fertilized eggs of rainbow trout, which are sensitive to molybdenum concentrations above $0.79 \mathrm{mg} / \mathrm{L}$ (Eisler, 1989).

'No Pennsylvania or federal criteria available. Value presented is the state of Colorado's hardness dependent water quality standard for the protection of aquatic life via chronic exposure (CDPHE, 1991).

fValue presented is the current safe level for molybdenum in cattle drinking water recommended by the U.S. Fish and Wildlife Service (Eisler, 1989).

Concentrations reported in milligrams per liter unless otherwise noted. Concentration in ground water is the upper confidence limit or maximum detected concentration, whichever is less.

L/kg - liters per kilogram.

$\mathrm{mg} / \mathrm{kg} \mathrm{DW}$ - milligrams per kilogram dry weight.

NA - not available. 


\section{Wildlife}

The exposure of terrestrial organisms from the ingestion of plants or animals that have accumulated contaminants (e.g., birds eating fish) is a potential exposure pathway at the site. Birds and other vertebrates consuming these plants and animals can bioaccumulate some of the contaminants of potential concern if the amount ingested exceeds the amount eliminated. This is often a function of the areal extent of contamination versus the areal extent of the animals feeding range. In the case of small contaminated areas, the contribution of impacted food to the total diet is usually very minute, and bioaccumulation is not a concern. Therefore, although exposure via the diet may be possible, the potential for bioaccumulation is not always a concern.

Biomagnification is potentially of greater concern and involves increased concentration of a constituent at each successive trophic level in the food chain. Of particular concern for biomagnification effects are the top predators, especially the carnivorous birds and mammals. Only a limited number of constituents have the potential for magnifying in the food chain. Most constituents are metabolized in organisms and eliminated at each level of the food chain and, thus, would not increase in concentration at each successive trophic level. Based on available information on the contaminants of concern at this site, the potential for these contaminants to represent a hazard via food chain transfer is probably low.

Other potential pathways of exposure to wildlife include ingesting water or sediments in Chartiers Creek. Animals may drink from the creek and, thus, be potentially exposed to contaminants in surface water and/or sediments. However, no available federal or state criteria or guidelines have been established to protect terrestrial wildlife from water or sediment exposure. Therefore, the potential hazards to terrestrial receptors from surface water and sediment ingestion were not evaluated.

\subsubsection{Aquatic risk}

\section{Surface water}

A potential exposure point for aquatic life is Chartiers Creek in the site vicinity. The ground water from the unconsolidated material is believed to discharge into the creek. Comparing the surface water data collected from the creek at the upstream location with those of the adjacent and downstream locations indicates that most of the constituents did not exceed upstream concentrations.

Manganese and molybdenum were identified as contaminants of potential concern in the creek water. The maximum concentrations for manganese $(0.17 \mathrm{mg} / \mathrm{L})$ and molybdenum $(0.17 \mathrm{mg} / \mathrm{L})$ were detected at sampling location 606 (Figure 7.1). No state or federal water quality criteria have been developed for these two constituents (PADER, 1991; 40 CFR Part 131). However, the EPA reported a value of $1.5 \mathrm{mg} / \mathrm{L}$ of manganese as the lower end of a tolerance 
range for freshwater organisms (EPA, 1986). The maximum detected manganese concentration in Chartiers Creek falls approximately 1 order of magnitude below this value. For molybdenum, the U.S. Fish and Wildlife Service currently recommends a value of $50 \mathrm{mg} / \mathrm{L}$ to protect most aquatic organisms and $0.79 \mathrm{mg} / \mathrm{L}$ to protect newly fertilized rainbow trout eggs (Eisler, 1989). Because Chartiers Creek is a warm water habitat, protecting trout eggs is not of concern. The maximum detected molybdenum concentration in Chartiers Creek falls well below the $50 \mathrm{mg} / \mathrm{L}$ value protective of aquatic organisms. The concentrations for manganese and molybdenum at sampling locations 606 and 605 were only slightly higher than those at the upstream location (Table 7.1). This suggests that ground water discharge to the creek has not affected the water quality, although the limited sampling is not conclusive.

\section{Sediment}

Surficial sediment (approximately 0 to 4 inches below sediment surface) was collected from six locations (Figure 7.1) during the 19 and 20 October 1993 sampling event. No sediment samples had been collected prior to this sampling event. The sediment data for manganese and molybdenum varied among sampling locations (Table 7.2). The highest concentration for each contaminant of potential concern was detected at a location adjacent to the site: location 604 for manganese and uranium and location 605 for molybdenum. The lowest concentration for each constituent was detected at location 602 .

No established state or federal sediment quality criteria or guidelines exist to protect aquatic life from the contaminants of potential concern at this site (EPA, 1988; NOAA, 1990). The EPA is evaluating a methodology based on the threephase sorption model for free metal ion activity and is assessing its applicability for determining the bioavailable fraction within sediments (EPA, 1989d). Currently, a number of other predictive models and methods are being investigated for metals, but no single approach has been accepted to adequately develop sediment-based metals criteria (Shea, 1988; Chapman, 1989; EPA, 1989d; NOAA, 1990; Di Toro et al., 1991; Burton, 1991).

Because no sediment quality values currently exist for manganese, molybdenum, and uranium, the potential for detected sediment concentrations to adversely affect biota could not be addressed. It is difficult to draw any conclusions concerning sediment quality conditions in Chartiers Creek due to the limited data set (i.e., one sampling event) and the variability of the data.

\subsection{LIMITATIONS OF THE ECOLOGICAL RISK ASSESSMENT}

The qualitative evaluation of potential ecological risks presented here is a screening level assessment of the risks associated with potential exposure of plants and animals to contaminated ground water, surface water, and sediment at the Canonsburg site. Sources of uncertainty in any ecological assessment arise from the monitoring data, exposure assessments, toxicological information, and the inherent complexities of the ecosystem. In addition, methods of 
predicting nonchemical stresses (e.g., drought), biotic interactions, behavior patterns, biological variability (i.e., differences in physical conditions, nutrient availability), and resiliency and recovery capacities are often unavailable. In general, limitations for the Canonsburg ecological risk assessment include the following:

- Only a small amount of ecological data were collected during this screening.

- Little is known about site-specific intake rates for wildlife or amounts of contaminants taken up by plants. General literature values were used in many cases.

- Only limited ecotoxicological reference data are available.

- Considerable uncertainty is associated with the toxicity of mixtures of contaminants.

\subsection{SUMMARY}

Surface water data from Chartiers Creek in the site vicinity indicate the presence of slightly higher concentrations for two constituents (manganese and molybdenum) at some downgradient locations. However, it is not known whether site-related constituents have affected the water quality of the Chartiers Creek.

A limited data set (one sampling round) currently exists of the sediment quality in Chartiers Creek in the site vicinity. Although a statistical analysis cannot be conducted with the limited data, no trends are apparent in the data. Sediment concentrations for the contaminants of potential concern were highest at two locations adjacent to the site. However, insufficient data are available to determine if this is related to releases from the site or due to natural variability. Sediment quality values to protect aquatic life are not available for the contaminants of potential concern; thus, it is not possible to evaluate the potential for these concentrations to represent an ecological concern.

Based on available data and criteria, no ecological threat exists to plants at harvestable maturity that may have roots in contact with soil saturated with the most contaminated ground water in the alluvial aquifer. Due to manganese levels, this ground water would not be suitable for continuous long-term irrigation for plants.

The potential for the contaminants of potential concern detected in media at the site to represent a food chain hazard (via bioaccumulation and biomagnification) is considered low, based on available surface water and sediment data. However, no tissue analysis from potential ecological receptors (e.g., wildlife and plants) has been conducted. 
Insufficient water quality and sediment quality values were available to allow a comprehensive evaluation of the impact of surface water, sediments, and contaminated ground water on ecological receptors. However, based on available data, it does not appear that former site activities have impacted surface water and sediments in the site vicinity. 


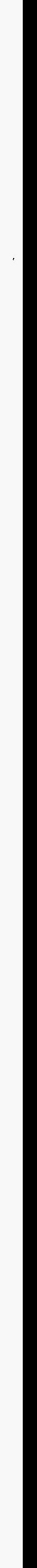




\subsection{INTERPRETATION AND RECOMMENDATIONS}

\subsection{RISK SUMMARY}

The UMTRCA requires the UMTRA Project to protect public health and the environment from radiological and nonradiological hazards associated with the uranium mill sites. This baseline risk assessment was conducted for the Canonsburg site to evaluate the presence of these hazards. This risk assessment is conservative in that it evaluates residential exposure by drinking water from the most contaminated well at the site. Because contaminated ground water is currently not used by area residents, human health is currently not at risk. This situation will continue if land and water use at the site remain the same. Changes of land use may or may not create future risks. Specific future land uses should be evaluated to identify potential health and environmental risks from the contaminated ground water in the unconsolidated materials (uppermost aquifer).

Future drinking water use of contaminated ground water could be associated with adverse health effects. However, it is unlikely that such use will occur in the future because the natural quality of the uppermost aquifer water is poor and this water has not been used for human consumption in the site area. Note also that in the future hypothetical residential scenario, only the people who drill a well in the most contaminated portion of the uppermost aquifer could experience the health problems discussed below. Furthermore, the ground water contaminant concentrations will decline over time.

The primary human health risk associated with ground water contamination in the uppermost aquifer at the Canonsburg site could be from manganese ingestion. Using ground water from the uppermost aquifer in Area $C$ for drinking purposes could result in ingesting manganese at levels that could most likely produce adverse effects to the nervous system. The estimated doses fall above the threshold for mild neurological symptoms in adults. Doses based on the natural background manganese levels detected in one well in the uppermost aquifer could also result in mild neurological symptoms. Manganese in ground water was also at levels that could be potentially harmful to ecological receptors. Ground water would not be suitable for continuous long-term irrigation for plants.

\subsection{LIMITATIONS OF THIS RISK ASSESSMENT}

Limitations to this evaluation of health risks follow:

- Most of the results presented here are based on 0.45 -micrometer filtered ground water samples. The effects of filtration differ for different elements. Filtered samples generally have lower concentrations of a given constituent than unfiltered samples because suspended particles are removed during filtration. However, constituents bound to these suspended particles can 
still produce toxic effects if ingested and broken down in the acid environment of the stomach.

- Potential exposure to and subsequent toxicity of a contaminant will vary from individual to individual. By using the probability distributions for potential exposure and presenting ranges of exposures that can produce toxic effects, this assessment attempts to emphasize that variability. However, it is not possible to account for all sources of variability and still present useful and meaningful analyses. Using ranges for expected toxic effects and distributions for expected exposures should provide the reader with a better understanding of the likelihood of toxic effects to occur.

- To assess toxicity, standardized reference values developed by various agencies, as well as toxicity data in the literature, were used. These data have limitations, including the following:

- Not all constituents elevated above background levels at a given site have comprehensive toxicity data available.

- In some cases, data obtained from laboratory animal testing at exposure doses different from those expected at the site were used to determine toxicity. The relationship between dose and response is not always linear, and humans do not always exhibit toxic effects similar to those seen in animals.

- Data used to determine toxicity are generally based on exposure to only one chemical. In reality, exposure to multiple chemicals occurs simultaneously. The interactive effects of multiple constituents and the impact of these interactions on expected toxicity generally cannot be accurately assessed from existing data.

- This document evaluates risks associated with exposures only to organic contaminants of ground water at the UMTRA Project site at Canonsburg. Potential organic contaminants related to uranium processing have not been considered.

- To assess potential intake via the fish ingestion exposure route, assumptions were made regarding uptake of contaminants into fish tissue. Uncertainties are associated with the BCFs used to make these estimates because these factors are taken from the literature and do not consider sitespecific conditions. In addition, data on the bioconcentration of molybdenum in fish were not available. Therefore, the fish ingestion pathway could not be quantitatively assessed for molybdenum.

- Although considerable effort has been directed at determining contaminant movement and placing monitor wells in locations that capture maximum contamination, variability in physical systems and models used to determine 
contaminant migration could still result in well placements that do not measure the highest contaminant concentrations or determine the fullest extent of contamination. On the other hand, because the major source of contamination (the tailings and contaminated soils) at the Canonsburg site has been removed, the assumption of a constant source used in this risk assessment may lead to an overestimation of risks. Because the contaminant concentrations could be higher or lower than those used in the analysis, the net effect of these uncertainties on future risk estimates cannot be predicted at this time.

- The drinking water pathway has been used as the major determinant of exposure. Although other pathways have been screened and determined not to contribute considerably to the total exposure, the additivity of exposure from these pathways should be kept in mind. Section 6.0 considers cases where a measurable contribution from other pathways could increase expected exposure enough to alter the predicted toxicity.

- The limitations for the Canonsburg ecological risk assessment include a limited amount of ecological data collected during this screening; little knowledge about site-specific intake rates for wildlife or amounts of contaminants taken up by plants; limited availability of ecotoxicological reference data; and considerable uncertainty associated with the toxicity of mixtures of contaminants.

By presenting ranges of toxic effects, probable exposure distributions, summaries of available data on health effects and interactions, and outlines of potential limitations, this document should provide a realistic interpretation of potential health risks associated with ground water contamination at this site. This assessment is designed to present a picture of contamination and risk as accurately as possible based on the available data, and to convey fields where uncertainties exist.

\subsection{GROUND WATER CRITERIA}

In 1983, the EPA established health and environmental protection standards for the UMTRA Project (40 CFR Part 192). The standards were revised and the final rule was published on 11 January 1995 (60 FR 2854). The ground water standards consist of ground water protection standards to evaluate disposal cell performance and ground water cleanup standards for existing contamination at processing sites. Concentration limits for constituents at the site are summarized in Table 8.1. Because maximum concentration limits (MCL) are not established for every contaminant, for contaminants without MCLs, background levels must be met. The standards also allow for supplemental standards or alternate concentration limits $(A C L)$ where appropriate.

In general, the EPA ground water standards are sufficient to protect humans and the environment. However, some risk assessments may identify site-specific factors that suggest these standards are not appropriate. When standards are 
Table 8.1 Concentration limits of constituents

\begin{tabular}{|c|c|c|c|}
\hline \multirow[b]{2}{*}{ Constituent } & $\begin{array}{c}\text { EPA MCL for } \\
\text { UMTRA } \\
40 \text { CFR } 192.02 \\
\end{array}$ & $\begin{array}{c}\text { Health advisories } \\
\text { 10-kg child, 10-daya }\end{array}$ & $\begin{array}{c}\text { Health advisories } \\
\text { 70-kg adult lifetime }\end{array}$ \\
\hline & \multicolumn{3}{|c|}{$(\mathrm{mg} / \mathrm{L})$} \\
\hline \multicolumn{4}{|l|}{ Chemicals (inorganic) } \\
\hline Antimony & NA & 0.01 & 0.003 \\
\hline Arsenic & 0.05 & NA & NA \\
\hline Barium & 1.0 & NA & 2 \\
\hline Boron & NA & 0.9 & 0.6 \\
\hline Cadmium & 0.01 & 0.04 & 0.005 \\
\hline Chromium & 0.05 & 1.0 & 0.1 \\
\hline Lead & 0.05 & NA & $0.015^{b}$ \\
\hline Mercury & 0.002 & NA & 0.002 \\
\hline Molybdenum & 0.1 & 0.04 & 0.04 \\
\hline Nickel & NA & 1.0 & 0.1 \\
\hline Nitrate (as $\mathrm{NO}_{3}{ }^{-}$) & $44^{c}$ & $44^{c}$ & NA \\
\hline Selenium & 0.01 & NA & NA \\
\hline Silver & 0.05 & 0.2 & 0.1 \\
\hline Strontium & NA & 25.0 & 17 \\
\hline Zinc & NA & 6.0 & 2 \\
\hline \multicolumn{4}{|l|}{ Radionuclides } \\
\hline Radium-226/-228 & $5 \mathrm{pCi} / \mathrm{L}$ & NA & NA \\
\hline $\begin{array}{l}\text { Uranium } \\
(U-234 /-238)\end{array}$ & $\begin{array}{c}30 \mathrm{pCi} / \mathrm{L} \\
(0.044 \mathrm{mg} / \mathrm{L})\end{array}$ & $0.03 \mathrm{mg} / \mathrm{L}^{\mathrm{d}, \mathrm{e}}$ & $0.1 \mathrm{mg} / \mathrm{L}^{d}$ \\
\hline
\end{tabular}

${ }^{\mathrm{a} E P A,} 1995$.

${ }^{b}$ Action level.

'Equals $10 \mathrm{mg} / \mathrm{L}$ nitrate as nitrogen.

${ }^{d}$ Proposed values (EPA, 1989e), under review; expected revision 1995.

'Longer-term health advisory.

NA - not available. 
too restrictive (for example, when there may be no potential for exposure), a less restrictive ACL may be sought. In other cases, the standards may not be sufficiently protective (for example, if many contaminants near the MCL have additive or synergistic adverse health effects).

While these standards apply only to the UMTRA Project, the EPA has also published drinking water health advisory levels for both long-and short-term exposures (EPA, 1995). Table 8.1 presents these advisories.

Contaminant concentrations in ground water from well 414 in Area C, which was used to assess potential risk in this assessment, do not exceed any of the EPA MCLs based on data since 1988. However, the uranium health advisory for children was exceeded once in the last sampling round in October 1993.

Due to permanent land use restrictions, no current or future point of potential exposure exists in the disposal site area (see Figure 2.2 for location). However, the three monitor wells 412 (unconsolidated unit), 413 (unconsolidated unit), and 506 (bedrock), all located within or just next to the restricted area, show uranium concentrations in excess of $\mathrm{MCL}$.

The molybdenum MCL was exceeded several times in downgradient wells, and the MCL was also exceeded in background wells. The lead MCL was exceeded a few times in both the unconsolidated and bedrock wells in 1987 and 1988. However, molybdenum and lead concentrations measured in subsequent years did not exceed their respective MCLs. The chromium MCL was also exceeded in unconsolidated and bedrock wells; however, this only occurred in 1988, and the MCL was also exceeded in a background well.

\subsection{RISK MITIGATION MEASURES}

Ground water from a deep bedrock aquifer which is not affected by the UMTRA Project site is used in the area for domestic purposes including drinking. No affected ground water is known to be used for any purposes. However, potential for adverse health effects to occur exists following long-term use of contaminated ground water from the uppermost aquifer below Area $C$ of the former Canonsburg uranium processing site. Although the cities of Canonsburg and Houston, North Strabane Township, and Chartiers Township are close to the processing site in Washington County, Pennsylvania, they are not expected to be influenced by ground water flow from the site. Ground water discharges into Chartiers Creek, which borders the western, northern, and eastern boundaries of the site. However, no site-related releases are known to affect the creek. The commonwealth of Pennsylvania water law is discussed below.

The Department of Environmental Resources Ground Water Quality Protection Strategy of the commonwealth of Pennsylvania describes the principles that the department intends to follow in implementing its ground water quality protection program through regulations, policies, and technical guidance. It states that the owner/operator of a property of concern is responsible for detecting and 
remediation and other applicable measures will be required. Applicable measures will include, but may not be limited to, treatment/containment of contaminated ground water as necessary to restore the ground water or to protect human health and the environment. The ultimate goal of ground water remediation is to reduce contamination levels to background quality. An owner/operator who restores contaminated ground water to its background quality will be released from further liability for remediation of those contaminants. If achieving background quality is not possible, the owner/operator will not be released from liability.

\subsection{RECOMMENDATIONS}

Ground water use is restricted on the majority of the Canonsburg site, with the potential exception of Area $\mathrm{C}$ which is being considered for public use. Based on the risk assessment results, the manganese levels in Area $C$ could result in potential adverse health effects following chronic exposures. Therefore, it is recommended that the contamination be better characterized prior to any future use of ground water beneath Area $C$.

Monitoring of ground water from the unconsolidated material, shallow bedrock, and potential surface expression points should continue until detailed characterization of the site ground water is complete. Such additional characterization will be discussed in future Canonsburg ground water documents.

Plant uptake studies are being conducted for the UMTRA Ground Water Project. Results of these studies will be used to evaluate the irrigated-produce and wildfruit-ingestion exposure pathway meaningfully. The results will be included in the NEPA document and ground water strategy planning for this site. 


\subsection{LIST OF CONTRIBUTORS}

The following individuals contributed to the preparation of this report.

\begin{tabular}{ll}
\hline Name & Contribution \\
\hline B. Malczewska-Toth & Overall document responsibility; toxicology \\
E. Timmer & Authorship \\
L. Flowers & Senior technical review \\
J. Weidner & Statistical evaluation \\
R. Heydenburg & Hydrogeology, surface water \\
D. Erskine & Geochemistry \\
M. Gawthrop-Cooper & Risk mitigation measures \\
S. Cox, J. Senger & Site management \\
L. Sanchez & Word processing \\
K. DeGruyter, B. Harvey, E. Bond & Graphic design \\
D. Kahl, K. Walston & Technical editing \\
D. Thalley & Document production coordination \\
\hline
\end{tabular}




\subsection{REFERENCES}

AQUIRE (Aquatic Information Retrieval), 1992. Computerized data base developed by the U.S. Environmental Protection Agency.

Alexander et al. (F. W. Alexander, B. E. Clayton, and H. T. Delves), 1974. Q.J. Med., Vol. 53, pp. 89-111.

Arnold, Mike, 1994. Waste Management Division, Environmental Resources Department, Commonwealth of Pennsylvania, personal communication with $M$. GawthropCooper, Regulatory Compliance Department, Jacobs Engineering Group, Inc., UPDCC File Location No. 1.19.7., UMTRA Project Office, Albuquerque Operations Office, Albuquerque, New Mexico, 2 June 1994.

Baes et al. (C. F. Baes, R. D. Sharp, A. J. Sjoreen, and R. W. Shor), 1984. A Review and Analysis of Parameters for Assessing Transport of Environmentally Released Radionuclides through Agriculture, ORNL-5786, prepared for Oak Ridge National Laboratory, Health and Safety Research Division, Oak Ridge, Tennessee.

Ballou et al. (J. E. Ballou, R. A. Gies, A. C. Case, D. L. Haggard, R. L. Buschbom, and J. L. Ryan), 1986. "Deposition and Early Disposition of Inhaled Uranium-233 Uranyl Nitrate and Uranium-232 Uranyl Nitrate in the Rat," Health Physics, Vol. 51, pp. 755-772.

Berlin, M., and B. Rudell, 1986. "Uranium," in Handbook on the Toxicology of Metals, second edition, L. Friberg, G. F. Nordberg, and V. B. Vouk, eds., pp. 647-658, Elsevier Science Publishers, New York, New York.

Brookins, D. G., 1988. Eh-pH Diagrams for Geochemistry, Springer-Verlag, Berlin, D. R. Burton, G. A., Jr., 1991, "Assessing the Toxicity of Freshwater Sediments," Environmental Toxicology and Chemistry, Vol. 10, No. 12, pp. 1585-1627.

Burton, G. A., Jr., 1991. "Assessing the Toxicity of Freshwater Sediments," Environmental Toxicology and Chemistry, No. 10, pp. 1585-1627.

Casarett, L. J., and J. Doull, 1991. Toxicology: The Basic Science of Poisons, fourth edition, M. O. Amdur, J. Doull, and C. D. Klaassen, eds., Pergamon Press, New York, New York.

CDPHE (Colorado Department of Public Health and Environment), 1991. Basic Standards and Methodologies for Surface Water, 3.1.0., Water Quality Control Commission, Denver Colorado.

Chapman, P. M., 1989. "Current Approaches to Developing Sediment Quality Criteria," Environmental Toxicology and Chemistry, Vol. 8, No. 7, pp. 589-599. 
Cothern, C. R., and W. L. Lappenbusch, 1983. "Occurrence of Uranium in Drinking Water in the U.S.," Health Physics, Vol. 45, pp. 89-99.

DHHS (U.S. Department of Health and Human Services), 1992. Toxicological Profile for Manganese, U.S. Department of Health and Human Services, Atlanta, Georgia.

DiToro et al. (D. M. DiToro, C. S. Zarba, D. J. Hansen, W. J. Berry, R. C. Swartz, C. E. Cowan, Sp. P. Pavlov, H. E. Allen, N. A. Thomas, and P. R. Paguin), 1991. "Technical Basis for Establishing Sediment Quality Criteria for Nonionic Organic Chemicals by Using Equilibrium Partitioning," Environmental Toxicology Chemistry, Vol 10, No. 12, pp. 1541-1583.

DOE (U.S. Department of Energy), 1995. "Supplement to the Baseline Risk Assessment of Ground Water Contamination at the Uranium Mill Tailings Site Near Canonsburg, Pennsylvania," DOE/AL/62350-149S, prepared by the U.S. Department of Energy, UMTRA Project Team, Albuquerque Operations Office, Albuquerque, New Mexico.

DOE (U.S. Department of Energy), 1994. Human Health Risk Assessment Methodology for the UMTRA Ground Water Project, DOE/AL/62350-170, prepared by the U.S. Department of Energy, UMTRA Project Office, Albuquerque Operations Office, Albuquerque, New Mexico.

DOE (U.S. Department of Energy), 1992. Surveillance and Maintenance Plan, Canonsburg, Pennsylvania, UMTRA-DOE/AL-350201.0000, prepared by the U.S. Department of Energy, UMTRA Project Office, Albuquerque Operations Office, Albuquerque, New Mexico.

DOE (U.S. Department of Energy), 1983a. Final Environmental Impact Statement, Remedial Actions at the Former Vitro Rare Metals Plant Site, Canonsburg, Washington County, Pennsy/vania, Volumes I and II, DOE/EIS-0096-F, prepared by the U.S. Department of Energy, UMTRA Project Office, Albuquerque Operations Office, Albuquerque, New Mexico.

DOE (U.S. Department of Energy), 1983b. Remedial Action Plan for Stabilization of the Inactive Uranium Mill Tailings Site at Canonsburg, Pennsylvania, UMTRA-DOE/AL-140, prepared by the U.S. Department of Energy, UMTRA Project Office, Albuquerque Operations Office, Albuquerque, New Mexico.

Eisler, R., 1989. "Molybdenum Hazards to Fish, Wildlife, and Invertebrates: A Synoptic Review," U.S. Fish and Wildlife Service, Contaminant Hazard Reviews, Report 19. U.S. Department of the Interior, Washington D.C.

EPA (U.S. Environmental Protection Agency), 1995. Drinking Water Regulations and Health Advisories, Office of Water, Washington, D.C. 
EPA (U.S. Environmental Protection Agency), 1994a. Integrated Risk Information System (IRIS) database, Office of Research and Development, Washington, D.C.

EPA (U.S. Environmental Protection Agency), 1994b. Health Effects Assessment Summary Tables (HEAST), Supplement No. 2, Office of Research and Development, Washington, D.C.

EPA (U.S. Environmental Protection Agency), 1993. Wildlife Exposure Factors Handbook, Vol. I, EPA/600/R-93/187a, Office of Research and Development, Washington, D.C.

EPA (U.S. Environmental Protection Agency), 1992. Drinking Water Criteria Document for Sulfate, final, Health and Environmental Criteria Division, Office of Science and Technology, U.S. Environmental Protection Agency.

EPA (U.S. Environmental Protection Agency), 1991. Risk Assessment Guidance for Superfund, Vol. I: Human Health Evaluation Manual; Supplemental GuidanceStandard Default Exposure Factors, OSWER Directive: 9285.6-03, Office of Emergency and Remedial Response, Washington, D.C.

EPA (U.S. Environmental Protection Agency), 1989a. Risk Assessment Guidance for Superfund, Vol. I, Human Health Evaluation Manual (Part A),

EPA/540/1-89/002, Office of Emergency and Remedial Response, Washington, D.C.

EPA (U.S. Environmental Protection Agency), 1989b. Exposure Factors Handbook, EPA/600/8-89/043, Office of Health and Environmental Assessment, Washington, D.C.

EPA (U.S. Environmental Protection Agency), 1989c. Risk Assessment Guidance for Superfund, Vol. II, Environmental Evaluation Manual, EPA/540/1-89/001, Office of Emergency and Remedial Response, Washington, D.C.

EPA (U.S. Environmental Protection Agency), 1989d. Briefing Report to the EPA Science Advisory Board on the Equilibrium Partitioning Approach to Generating Sediment Quality Criteria, Office of Water Regulations and Standards, Criteria and Standards Division, Washington, D.C.

EPA (U.S. Environmental Protection Agency), 1989e. Drinking Water Criteria Document for Uranium, external review draft, Criteria and Standards Division, Office of Drinking Water, Washington, D.C.

EPA (U.S. Environmental Protection Agency), 1988. Interim Sediment Criteria Values for Nonpolar Hydrophobic Organic Contaminants, Office of Water Regulations and Standards Division, Washington, D.C. 
EPA (U.S. Environmental Protection Agency), 1986. "Quality Criteria for Water, 1986," U.S. Environmental Protection Agency, Office of Water, Regulations, and Standards, Washington, D.C.

EPA (U.S. Environmental Protection Agency), 1972. "Water Quality Criteria," National Academy of Sciences and National Academy of Engineering, Washington, D.C.

FBDU (Ford, Bacon, and Davis Utah, Inc.), 1982. Engineering Assessment of Inactive Uranium Mill Tailings, Canonsburg Site, Canonsburg, Pennsy/vania, DOE/UMT-0101, Salt Lake City, Utah.

Fisenne et al. (I. M. Fisenne, P. M. Perry, and N. H. Harleyl, 1988. "Uranium in Humans," Radiation Protection Dosimetry, No. 24, pp. 127-131.

Friberg et al. (L. Friberg, G. F. Nordberg, and V. B. Vouk), 1986. Handbook on the Toxicology of Metals, Volume II: Specific Metals, second edition, Elsevier, New York, New York.

Gilman et al. (A.G. Gilman, L.S. Goodman, and A. Gilman), 1990. The Pharmacological Basis of Therapeutics, sixth edition, Macmillan Publishing Company, Inc., New York, New York.

Goyer, R. A., 1991. "Toxic Effects of Metals," in Toxicology: The Basic Science of Poisons, fourth edition, M. O. Amdur, J. Doull, and C. D. Klaassen, eds., Pergamon Press, New York, New York, pp. 623-680.

Hull, R. N., and G. W. Suter, 1994. Toxicological Benchmarks for Screening Contaminants of Potential Concern for Effects on Sediment-Associated Biota: 1994 Revision, ES/ER/TM-95/RI, U.S. Department of Energy, Oak Ridge National Laboratory, Oak Ridge Tennessee.

JEG (Jacobs Engineering Group Inc.), n.d. Albuquerque Operations Manual, standard operating procedures, prepared by Jacobs Engineering Group Inc., prepared for the U.S. Department of Energy, Environmental Restoration Division, UMTRA Project Team, Albuquerque, New Mexico.

Kabata-Pendias, A., and H. Pendias, 1992. Trace Elements in Soils and Plants, second edition, CRC Press, Boca Raton, Florida.

Kondakis et al. (X. G. Kondakis, N. Makris, M. Leotsinidis, M. Prinou, and R. Papapetropoulos), 1989. "Possible Health Effects of High Manganese Concentrations in Drinking Water," Archives of Environmental Health, Vol. 44, No. 3, pp. 175-178.

Langmuir D. and J. S. Herman, 1980. "The Mobility of Thorium in Natural Waters at Low Temperatures," in Geochem. Cosmochim. Acta, Vol. 44, pp. 1753-1766. 
Latarjet, R., 1992. "Radiation Carcinogenesis and Radiation Protection," in The Cancer Journal, Vol. 5, No. 1, pp. 23-27.

Lazo, T.,1994. "French Researcher Suggests Existence of a Threshold for Cancer Induction," Health Physics Society Newsletter, Vol. 22, No. 1, pp. 5-6.

Leach et al. (L. J. Leach, R. M. Gelein, B. J. Panner, C. L. Ylie, and C. C. Cox), 1984. The Acute Toxicity of the Hydrolysis Products of Uranium Hexafluoride (UF6) When Inhaled by the Rat and Guinea Pig, final report, ISS K/SUB-81-9039-3, National Technical Information Service, Springfield, Virginia.

Licensing Implementation Plan, 1992. "Licensing Implementation Plan for Canonsburg, Pennsylvania, Disposal Site and Burrell, Pennsylvania, Vicinity Property Disposal Cell," with letter dated 18 May 1992, from Albert R. Chernoff, UMTRA Project Office, to John J. Surmeier, U.S. Nuclear Regulatory Commission.

Lönnerdal et al. (B. Lönnerdal, C. L. Keen, J. G. Bell, and B. Sandstrom), 1987. "Manganese Uptake and Retention," Nutritional Bioavailability of Manganese, C. Kies, ed., American Chemical Society, symposium series 354, pp. 9-20, American Chemical Society, Washington, D.C.

Maynard, E. A., and H. C. Hodge, 1949. "Study of Toxicity of Various Uranium Compounds When Fed to Experimental Animals," Pharmacology and Toxicology of Uranium Compounds, National Nuclear Energy Services, Div. VI, Vol. 1, pp. 309-376, C. Voegtlin and H. C. Hodge, eds., McGraw Hill, New York, New York.

Mena et al. (I. Mena, K. Horiuchi, K. Burke, and G. C. Cotzias), 1969. "Chronic Manganese Poisoning, Individual Susceptibility and Adsorption of Iron," Neurology, No. 19, pp. 1000-1006.

MK-F (MK-Ferguson), 1986. Canonsburg, Uranium Mill Tailings Site Remedial Action Completion Report, prepared by MK-Ferguson for the U.S. Department of Energy, UMTRA Project Office, Albuquerque Operations Office, Albuquerque, New Mexico.

National Research Council, 1973. Medical and Biological Effects of Environmental Pollutants: Manganese, Committee on Biologic Effects of Atmospheric Pollutants, Division of Medical Sciences.

NCRP (National Council on Radiation Protection and Measurements), 1984. Exposures from the Uranium Series with Emphasis on Radon and its Daughter, NCRP Report No. 77, Bethesda, Maryland.

Newport, T. G., 1973. Summary, Ground-Water Resources of Washington County, Pennsy/vania, Water Resource Report 38, Pennsylvania Geological Survey, Harrisburg, Pennsylvania. 
NOAA (National Oceanic and Atmospheric Administration), 1990. "The Potential for Biological Effects of Sediment-Sorbed Contaminants Tested in the National Status and Trends Program." NOAA Technical Memorandum NOS OMA 52 , Seattle, Washington.

Oftedal, P., 1989. "A Holistic View of Low-Level Radiation Effects in Biological Systems," Canadian Journal of Physics, Vol. 68, pp. 974-978.

PADER (Pennsylvania Department of Environmental Resources), 1992. Water Quality Standards, Chapter 93, Title 25, Environmental Resources, Pennsylvania Code.

PADER (Pennsylvania Department of Environmental Resources), 1991. Water Quality Criteria for Toxic Substances, Chapter 16.51, Title 25, Environmental Resources, Pennsylvania Code.

Palisade Corporation, 1992. @ RISK, Risk Analysis and Simulation Add-In for Lotus 1-2-3, Version 2.01, Newfield, New York.

Parkhurst et al. (D. L. Parkhurst, D. C. Thorstenson, and D. N. Plummer), 1980. PHREEQEÄA Computer Program for Geochemical Calculations, U.S. Geological Survey, Water Resources Investigation 80-96, National Technical Information Services Report PB81-167-801, Springfield, Virginia.

PGS (Pennsylvania Geologic Survey), 1994. "Information Concerning the Pennsylvania Geologic Survey's Water Well Inventory Data," UPDCC File Location No. 1.19.7, UMTRA Project Office, Albuquerque Operations Office, Albuquerque, New Mexico.

Power, E. A., and P. M. Chapman, 1992. "Assessing Sediment Quality," in Sediment Toxicity Assessment, G. A. Burton, Jr., ed., Lewis Publishers.

PNL (Pacific Northwest Laboratory), 1989. "Sorption Distribution Coefficient Data," Multimedia Environmental Pollutant Assessment, Pacific Northwest Laboratory, Richland, Washington.

Prister, B. S., 1969. GKIAE Report by Atomizdat, Moscow (Canadian translation AEC/TR/7178 (1970), and USCEAR Rep. A/AC.82/G/L 1298).

Rigaud et al. (O. Rigaud, D. Papadopoulo, and E. Moustacchi), 1993. “Decreased Deletion Mutation in Radiolabeled Human Lymphoblasts," Radiation Research, 133, pp. 94-101.

Robinson et al. (M. F. Robinson, J. M. McKenzie, C. Thomson, and A. L. VanRijn), 1973. Br. J. Nutri, Vol. 30, pp. 195-205.

Roseberry, A. M., and D. E. Burmaster, 1992. "Lognormal Distributions for Water Intake by Children and Adults," Risk Analysis, Vol. 12, No. 1, pp. 99-104. 
Rosoff, B., and H. Spencer, 1964. Nature (London), Vol. 202, pp 410-411.

Saric, M., 1986. "Manganese," Handbook on the Toxicology of Metals, Vol. II, pp. 354-386. L. Friberg, G. F. Nordberg, V. B. Vouk, eds., Elsevier, New York.

Shadley, J. D., and J. K. Wiencke, 1989. "Induction of the Adaptive Response by X-rays is Dependent on Radiation Intensity," International Journal of Radiation Biology, Vol. 56, No. 1, pp. 107-118.

Shea, D., 1988. "Developing National Sediment Quality Criteria," Environmental and Scientific Technology, Vol. 22, No. 11, pp. 1256-1261.

Shleien, B., 1992. The Health Physics and Radiological Health Handbook, revised edition, Scinta, Inc., Silver Spring, Maryland.

Stevens et al. (W. F. Stevens, W. Bruenger, D. R. Atherton, J. M. Smith, and

G. N. Taylor), 1980. "The Distribution and Retention of Hexavalent ${ }^{233} \mathrm{U}$ in the Beagle," Radiation Research, Vol. 83, pp. 109-126.

Sullivan et al. (M. F. Sullivan, P. S. Ruemmler, J. L. Ryan, and R. L. Bushhom), 1986. "Influence of Oxidizing or Reducing Agents on Gastrointestinal Absorption of $U$, Pu, Am, Cm, and Pm by Rats," Health Physics, Vol. 50, pp. 223-232.

Summerskill W. H. H., and E. Wolpert, 1970. "Ammonia Metabolism in the Gut," American Journal of Clinical Nutrition, Vol. 23, pp. 633-639.

TAC (Technical Assistance Contractor), 1994a. "Summary of the Site-Related Contamination at Canonsburg, Rationale for Selection of Sampling Dates, and Development of List of Contaminants of Potential Concern," unpublished report prepared by the TAC, Jacobs Engineering Group Inc., for the U.S. Department of Energy, UPDCC File Location No. 1.19.7, UMTRA Project Office, Albuquerque Operations Office, Albuquerque, New Mexico.

TAC (Technical Assistance Contractor), 1994b. Meeting notes, 28 February 1994, regarding Canonsburg site status, available in the UMTRA Project Document Control Center, UPDCC File Location No 1.24.1.4, UMTRA Project Office, Albuquerque Operations Office, Albuquerque, New Mexico.

TAC (Technical Assistance Contractor), 1993. "Unpublished Field Notes, Canonsburg, Pennsylvania, Uranium Mill Tailings Site," unpublished report prepared by the TAC, Albuquerque, New Mexico, for the U.S. Department of Energy, UMTRA Project Office, Albuquerque Operations Office, Albuquerque, New Mexico.

TAC (Technical Assistance Contractor), 1990. "Unpublished Field Notes, Canonsburg, Pennsylvania, Uranium Mill Tailings Site," unpublished report prepared by the TAC, Albuquerque, New Mexico, for the U.S. Department of Energy, UMTRA Project Office, Albuquerque Operations Office, Albuquerque, New Mexico. 
Taylor, Samuel, 1994. Customer Service Representative, Pennsylvania-American Water Company, personal communication with E. Timmer and B. Toth, Data Management and Assessment Department, Jacobs Engineering Group Inc., UPDCC File Location No. 1.19.7, UMTRA Project Office, Albuquerque Operations Office, Albuquerque, New Mexico.

Templeton, D., 1993. "Trash, Debris, Slabs at Creek Pose Safety Hazard, Agency Told," Pittsburgh Post-Gazette, Sunday, March 28, 1993.

Tracy et al. (B. L. Tracy, F. A. Prantl, and J. M. Quinn), 1983. "Transfer of ${ }^{226} \mathrm{Ra}{ }^{210} \mathrm{~Pb}$, and Uranium from Soil to Garden Produce: Assessment of Risk," Health Physics, Vol. 44, p. 469.

Tracy et al. (B. L. Tracy, J. M. Quinn, J. Lahey, A. P. Gioman, K. Mancuso, A. P. Yagdinas, and D. C. Villeneuve), 1992. "Absorption and Retention of Uranium from Drinking Water by Rats and Rabbits," Health Physics, Vol. 62, pp. 65-73.

Washington County Board of Commissioners, 1993. Industrial Directory of Washington County, Pennsy/vania. 1992-93.

Washington County Planning Commission, 1993. Washington County Profile.

Weigel, F., 1983. "Uranium and Uranium Compounds," in Kirk-Othmer Encyclopedia of Chemical Technology, Vol. 23, third edition, pp. 502-547, M. Grayson, ed., John Wiley and Sons, New York, New York.

Wrenn et al. (M. E. Wrenn, P. W. Durbin, B. Howard, J. Lipsztein, J. Rundo, E. T. Still, and D. L. Willis), 1985. "Metabolism of Ingested U and Ra," Health Physics, Vol. 48, pp. 601-633.

\section{CODE OF FEDERAL REGULATIONS}

10 CFR Part 40, Domestic Licensing of Source Materials, U.S. Nuclear Regulatory Commission.

40 CFR Part 131, Water Quality Standards; Establishment of Numeric Criteria for Priority Toxic Pollutants; States' Compliance; Final Rule, U.S. Environmental Protection Agency.

40 CFR Part 192, Health and Environmental Protection Standards for Uranium and Thorium Mill Tailings, U.S. Environmental Protection Agency. 


\section{FEDERAL REGISTER}

60 FR 2854, Groundwater Standards for Remedial Actions at Inactive Uranium Processing Sites, final rule, U.S. Environmental Protection Agency, 11 January 1995.

\section{UNITED STATES CODE}

42 USC $§ 7901$ et seq., Uranium Mill Tailings Radiation Control Act, 8 November 1978.

42 USC $\$ 7922$ et seq. . Uranium Mill Tailings Remedial Action Amendments Act, 5 November 1988. 
